\title{
GEOMETRIC, ALGEBRAIC, AND ANALYTIC DESCENDANTS OF NASH ISOMETRIC EMBEDDING THEOREMS
}

\author{
MISHA GROMOV
}

Abstract. Is there anything interesting left in isometric embeddings after the problem had been solved by John Nash? We do not venture a definite answer, but we outline the boundary of our knowledge and indicate conjectural directions one may pursue further.

Our presentation is by no means comprehensive. The terrain of isometric embeddings and the fields surrounding this terrain are vast and craggy with valleys separated by ridges of unreachable mountains; people cultivating their personal gardens in these "valleys" only vaguely aware of what happens away from their domains and the authors of general accounts on isometric embeddings have a limited acquaintance with the original papers. Even the highly cited articles by Nash have been carefully read only by a handful of mathematicians.

In order not to mislead the reader, we try be open about what we do and what we do not know firsthand and to provide references to what is missing from the present paper.

\section{Contents}

1. Isometric Embeddings $X^{n} \rightarrow \mathbb{R}^{q}$ According to John Nash

1.1. Three Isometric Embedding Theorems

1.2. Twelve Sample Questions and Conjectures

2. Where Is the New Land?

2.1. Waring Connection

2.2. Infinite Dimensional Representations of Diff, Invariant Convex Cones and Permutations of Primes

2.3. Maps Between Manifolds, Induced Structures, Composability, and Functoriality

2.4. Homotopy Theoretic Perspective on PDE and PDR

2.5. Stephen Smale and the $h$-Principle

3. Basic Constructions

3.1. Kuratowski-Weyl Embeddings and Nash Spherical Decomposition

3.2. Adding $d \phi^{2}$ to Induced Riemannian Metrics $g$ by $C^{1}$-Twist and the

\section{Proof of Nash's $C^{1}$-Immersion Theorem}

3.3. Kuiper's $C^{1}$-Stretch and Isometric $C^{1}$-Immersions $X^{n} \rightarrow Y^{n+1}$

3.4. The Proof of the Smale-Hirsch $h$-Principle by Nash-Kuiper Stretching 194

3.5. Convex Integration

3.6. Solving Differential Equations by Algebraic Formulas 207

3.7. Nash Implicit Function Theorem 
Analytic Perturbations

Local Immersions by Perturbation + Scaling

Immersions to non-Euclidean manifolds $Y^{q}=\left(Y^{q}, \tilde{g}\right)$.

Elliptic Immersions of Surfaces with Codimensions $\geq 1$

3.8. Nash Decomposition, Conformal Twist, and $C^{\infty}$-Approximate Embeddings

3.9. Nash Twist in non-Riemannian Categories

3.10. Microflexibility, Flexibility, and the $h$-Principle

3.11. Local Janet's Equations and Semilocal Extension of Isometric Immersions.

3.12. Addition of $d \phi^{2}$ by $C^{\infty}$-Twist, Semilocal Immersions, and Global Cylinders

4. Production of Problems and Classification of Structures

4.1. Categories, Sheaves, Jets, and $h$-Principles

Topological Obstructions to Isometric Immersions

4.2. Trends in Structures

Isometric Immersions of Higher Order

On Higher Dimensional Inheritance

4.3. Unclassified Problems and Conjectures

\section{Isometric Embeddings $X^{n} \rightarrow \mathbb{R}^{q}$ According to John Nash}

In 1954-1966 Nash discovered several new constructions of isometric embedding: 1 from Riemannian $n$-manifolds $X=(X, g)$ to the Euclidean spaces $\mathbb{R}^{q}$ for some universal $q=q(n)$.

Using these constructions, he proved the following.

\subsection{Three Isometric Embedding Theorems.}

$C^{1}$-Theorem. $C^{0}$-Riemannian $n$-manifolds (i.e., with continuous Riemannian metrics) admit isometric $C^{1}$-embeddings into the Euclidean space $\mathbb{R}^{2 n}$. ( $C^{1}$-isometric imbeddings. Ann. Math. 60 (1954), 383-396 [62]).

Smooth Embedding Theorem. Compact $C^{r}$-Riemannian $n$-manifolds for $r=$ $3,4, \ldots, \infty$ admit isometric $C^{r}$-embeddings to $\mathbb{R}^{q}$ for $q=3 s_{n}+4 n$, where $s_{n}=\frac{n(n+1)}{2}$, and non-compact ones admit such embeddings to $\mathbb{R}^{q}$ for $q=(n+1)\left(3 s_{n}+4 n\right)$.

(The imbedding Problem for Riemannian manifolds. Ann. Math. 63 (1956), 20-63 63]).

\footnotetext{
1 "Isometric" here means preserving the lengths of the curves. If such an isometric map $f: X \rightarrow$ $Y$ is $C^{1}$-smooth, then it is necessarily a smooth immersion, i.e., its differential $D f$ is injective on all tangents spaces $T_{x}(X)$.

Equivalently, an isometric immersion between Riemannian manifolds, $f:(X, g) \rightarrow(Y, h)$, is a $C^{1}$-smooth map, such that the (quadratic differential) form $(D f)^{\star}(h)$ on $X$ induced from $h$ by the differential of $f$ equals $g$.

The relation $(D f)^{\star}(h)=g$ written in local coordinates becomes a system of $s_{n}=\frac{n(n+1)}{2}$, $n=\operatorname{dim}(X)$, first order partial differential equations in $q=\operatorname{dim}(Y)$ unknown functions on $X$; being a topological embedding, i.e., beng a homeomorpism onto its image, is of secondary importance for these $f$.
} 
$C^{a n}$-Embedding Theorem.2 Compact real analytic Riemannian $n$-manifolds admit isometric real analytic embeddings to $\mathbb{R}^{q}$ for the same $q=3 s_{n}+4 n$.

(ANALYTICITY OF THE SOLUTIONS OF IMPLICIT FUNCTION PROBLEMS WITH ANALYTiC DATA. Ann. Math. 84 (1966), 345-355 64]).

Nash's solution of the long-standing isometric embedding problem had galvanised the mathematical community, but the reaction to the new concepts and ideas behind these theorems was far from uniform.

The general principles underlying Nash's constructions suggested a transplantation of isometric immersions to a land of a new kind of mathematics positioned far away from classical differential geometry and analysis, and foreign to most mathematicians of 1950 s.

Unsurprisingly, it took about 15 years for the global geometry and geometric topology community to achieve the level of maturity needed to absorb and to allow a development of the most profound and innovative ideas ingrained in Nash's constructions.

On the other hand analytically trained mathematicians, who regarded Nash's paper to be "difficult" (some insist on this until the present day), have focused on Nash's technical - let them be amazingly fecund and versatile - lemmas, notably on his perturbation, also called implicit function, theorem, and kept rewriting Nash's proofs (often incomplete fragments of these) in the traditional language 3

But if you read Nash's writings in earnest, the mirage of difficulties disappears and new vistas open before your eyes; much of what was dark and obscure becomes obvious, e.g., the beautifully transparent logic of his proof of the implicit function/perturbation theorem that is better adapted to geometric applications than its later renditions.

The main goal of the present article is a general introduction to remaining OPEN PROBLEMs, where, to be self-contained, we reproduce all background definitions, propositions and non-technical arguments. For more general results and detailed proofs, we refer to the corresponding sections in [35, my 1986 Springer book Partial Differential Relations, which contains an extended account on the work of Nash.

Many of these problems can, probably, be solved following in the steps of Nash, but the most intresting among them need an influx of new ideas.

\subsection{Twelve Sample Questions and Conjectures.}

Nash's dimensions $q>3 s_{n}$ and especially his $q>(n+1) s_{n}$ for non-compact manifolds are extravagantly large if compared with the local case, where one has the following

$C^{a n}$-Local Theorem of Janet, Cartan, and Burstin. $C^{a n}$-Riemannian $n$ manifolds admit loca 4 isometric $C^{a n}$-embeddings to $\mathbb{R}^{s_{n}}$.

It still remains problematic, even for $n=2$, whether a similar result holds for $C^{\infty}$-metrics - this is called the $C^{\infty}$-local Schläfli conjecture-but definitely one cannot go below $q=s_{n}$.

\footnotetext{
${ }^{2} C^{a n}$ stands for real analytic.

${ }^{3}$ Contrary to what you often hear, nobody has managed to improve or to simplify the analytic results obtained by Nash. But Nash's style smoothing techniques were remarkably applied by Jürgen Moser to the KAM theory of Hamiltonian systems - subject matter I am not qualified to discuss.

4 "Local" means in a small neighbourhood of a given point.
} 
Generically (and rather obviously), real analytic Riemannian $n$-manifolds admit no sufficiently smooth5 local isometric immersions to $\mathbb{R}^{q}$ for $q<s_{n}$.

But if $q>s_{n}$, one expects (isn't it too "optimistic"?) that smooth isometric immersions $X^{n} \rightarrow \mathbb{R}^{q}$ are (almost) as abundant as in the much larger dimensions where they were constructed by Nash.

For instance, taking some topological precautions (that may or may not be necessary), we suggest the following

$2 \quad\left(s_{n}+1\right)$-Conjecture $6 C^{r}$-smooth parallelisable (e.g., diffeomorphic to the $n$ tori) Riemannian $n$-manifolds, $n \geq 2$, admit isometric $C^{r}$-immersions to $\mathbb{R}^{q}$ for $q=s_{n}+1$ and all $r=1,2,3, \ldots, \infty$, an.

Moreover, there is a stronger version of this conjecture that does not even need the concept of a Riemannian manifold which is formulated below in the real analytic case.

Let $\left\{\tau_{i}\right\}_{i=1, \ldots, n}$ be a frame of linearly independent $C^{a n}$-vector fields on an $N$ dimensional manifold $X$.

Orthogonal Frame Conjecture. There exists a real analytic map $f: X \rightarrow \mathbb{R}^{q}$ for $q=s_{n}+1=\frac{n(n+1)}{2}+1$, such that the images of these fields under the differential $D f: T(X) \rightarrow T\left(\mathbb{R}^{q}\right)$ are orthonormal at all $x \in X$.

The smallest $q$ available today for 2 is $q=\frac{(n+1)(n+2)}{2}+4=s_{n}+n+5$ for $C^{\infty}$ and $C^{a n}$-manifolds, due to Mattias Günther (1989) [41] [42.7 while "addition of $d \phi^{2}$ by Nash twist" from 3.12 applies in the context of 3 and delivers the proof for $q=s_{n+2}=s_{n}+2 n+3$.

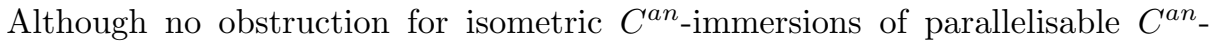
Riemannian $n$-manifolds to $\mathbb{R}^{s_{n}}$ for $n>2$ is anywhere in sight yet, it seems probable that

4 generic analytic perturbations of the standard flat metric on the $n$-torus $\mathbb{T}^{n}$ admit no isometric $C^{a n}$-immersions to $\mathbb{R}^{s_{n}}$.

Yet, at the present day, one can neither rule out the possibility that small analytic perturbations $g_{\varepsilon}$ of the flat metric on $\mathbb{T}^{3}$ can be induced by analytic perturbations of the standard embedding $\mathbb{T}^{3} \subset \mathbb{R}^{6}$ nor show that $g_{\varepsilon}$ can be induced by perturbations of this embedding in the ambient space $\mathbb{R}^{7} \supset \mathbb{R}^{6} \supset \mathbb{T}^{3}$.

(The former is an analytic problem lying beyond our present means, while the latter, probably, may be resolved by purely algebraic means; see section 3.6.2.)

\footnotetext{
${ }^{5} \mathrm{~A}$ rough counting-parameters argument shows that $C^{r}$ for $r>\left(s_{n}-1\right) n+1$ is smooth enough for this purpose, where a better bound on $r$ follows from the Gauss formula.

${ }^{6}$ The existence of smooth isometric immersions of (compact?) surfaces to $\mathbb{R}^{4}$ was proposed as a conjecture by Chern around 1950 .

${ }^{7}$ In general, with no parallelisability assumption, Günther constructs his isometric $C^{\infty}$ embeddings $X^{n} \rightarrow \mathbb{R}^{q}$ for $q=q_{g u n}=s_{n}+2 n=\frac{n(n+1)}{2}+2 n$. But since I failed to penetrate Günther's analytic writing, the best I can vouch is $q=s_{n+2}=s_{n}+2 n+3$ (see 3.1.7 in 35] 1986).

Notice also, that Günther carries out his construction only for $C^{\infty}$-metrics, while the construction from 35 allows $C^{r, \alpha}$-Hölder embedding of $C^{r, \alpha}$-metrics for all $r \geq 4$ and $\alpha>0$ (including plain $C^{r}$ for $r \geq 5$ and $\left.C^{a n}\right)$. But $\left(X^{n}, g\right) \mapsto \mathbb{R}^{q}$ embedding of $C^{r, \alpha}$-metrics $g$ with $2<r+\alpha \leq 4$ (including $C^{3}$ and $C^{4}$ ) are available by perturbation+embedding theorems from 35, only for $q=\left(s_{n}+2 n\right)+\left(s_{n}+2 n+3\right)=n^{2}+5 n+3$ where " 3 " can be removed with $q_{g u n}=s_{n}+2 n$ and where one can go down to $q=\left(s_{n}+n+1\right)+\left(s_{n}+n+5\right)$ for parallellisable manifolds $X$.
} 
Amazingly,

the minimal $q$ is unknown even for smooth isometric immersions of

(non-split!) flat $n$-tori to $\mathbb{R}^{q}$.

(Compact flat $n$-manifolds $X$ admit no isometric $C^{2}$-immersions $X \rightarrow \mathbb{R}^{2 n-1}$, while the smallest known $q$ where all such manifolds $X$ embed is $q=s_{n}+\operatorname{int}\left[\frac{1}{2} n\right]$; see 3.12.)

Outrageous $C^{2}$-Immersion Conjecture. By his construction of isometric $C^{1}$-embeddings, Nash demonstrated, contrary to conventional wisdom, that regularity required of an isometric embedding is an essential condition rather than a technicality.

But does something of the kind happen for regularity classes (significantly) above $C^{1}: 8$

For example:

do there exist, for a given $r=2,3,4, \ldots$, real analytic or $C^{\infty}$-smooth Riemannian manifolds $X$ of dimension $n$ that admit isometric $C^{r}$-immersions to $\mathbb{R}^{q_{r}}$ for some $q_{r}=q_{r}(X)$ but no isometric $C^{\text {an }}$ - or $C^{\infty}$-immersions to $\mathbb{R}^{q}$ for $q \leq\left(1+c_{r}\right) q_{r}$ for some $c_{r}>0$ and all sufficiently large $n$ ?

Are generic $C^{a n}$-manifolds like that?

If so, then they would be isometrically $C^{r}$-immersible to $\mathbb{R}^{q}$ for $q$ significantly smaller than $s_{n}$ for large $n$.

But how could it be, for instance, no matter what $n$ is, that

all (at least "reasonably generic") $C^{\text {an }}$-manifolds would admit isometric (let them be local) $C^{2}$-immersions to $\mathbb{R}^{q}$ for $q<s_{n}$ ?

If this is impossible, then the gap discussion for general metrics collapses to nothing, but...

there are no known obstructions to local isometric $C^{2}$-immersions $(X, g) \rightarrow \mathbb{R}^{q}$ except those implied by the Gauss formula expressing the (intrinsic) curvature of $g$ by the (extrinsic) curvature of $f$.

Now, the curvature of $g$ has $\frac{n^{2}\left(n^{2}-1\right)}{12} \approx \frac{s_{n}^{2}}{3}$ components, while the (extrinsic) curvature of $f$ that is the (second) quadratic form on $X$ with values in the normal bundle of $X \underset{f}{\subset} \mathbb{R}^{q}$ has $s_{n}(q-n)$ components.

Since the Gauss formula is invariant under orthogonal transformations of the normal spaces of $X \underset{f}{\subset} \mathbb{R}^{q}$, the number of relevant parameters reduces to $s_{n}(q-n)-\frac{(q-n)(q-n+1)}{2}$.

This shows that if $s_{n}(q-n)-\frac{(q-n)(q-n+1)}{2}<\frac{n^{2}\left(n^{2}-1\right)}{12}$, then generic smooth (or real analytic, if you wish) Riemannian $n$-manifolds admit no isometric $C^{2}$-immersions to $\mathbb{R}^{q}$.

This also suggests that if $s_{n} q-\frac{q^{2}}{2}$ is "significantly greater" than $\frac{s_{n}^{2}}{3}$ (that is if $\left.q \gg s_{n}\left(1-\frac{1}{\sqrt{3}}\right)\right)$, then all curvature tensors at a point $x_{0} \in X$ come from curvatures of maps $X \rightarrow \mathbb{R}^{q}$. This seems an easy algebraic problem but, if true, it may (?) lead to something more interesting.

\footnotetext{
${ }^{8} C^{1, \alpha}$-Hölder maps for smallish $\alpha$ are studied from this angle in [7], 8], [52, [15] (we return to this in 3.5.5); and see [1, 55, 56, 65] for less than $C^{1}$-regular maps.
} 
$9 \quad$ Conjecture. If $q \gg\left(1-\frac{1}{\sqrt{3}}\right) s_{n}$ where, for safety's sake, this relation is interpreted here as

$$
q>\left(1-\frac{1}{2 \sqrt{3}}\right) s_{n}+n \approx 0.36 n^{2}+1.36 n,
$$

then all $C^{\infty}$-smooth $\left(C^{2}\right.$-smooth?) Riemannian $n$-manifolds admit local (global?) isometric $C^{2}$-immersions to $\mathbb{R}^{q}$.

Of course, looking from the classical perspective, one would expect that, for all $n$, only very special metrics on $n$-manifolds admit isometric $C^{2}$-immersions to $\mathbb{R}^{s_{n}-1}$.

\section{$C^{2}$-Immersions with Prescribed Curvatures}

The Nash $C^{1}$-embedding theorem has the following counterpart for $C^{2}$-immersions with prescribed curvatures (see 3.1.5 in [35) that offers "non-classical evidence" in favour of something even stronger than the outrageous $C^{2}$-conjecture:

Let the extrinsic curvature of a $C^{2}$-immersion $X \rightarrow \mathbb{R}^{q}$ be defined as a function on the tangent bundle of $X$, say $K^{+}=K^{+}(\tau), \tau \in T_{x}(X), x \in X$, such that

$$
K^{+}(\tau)=\|\tau\|^{4} \operatorname{curv}^{2}(\tau),
$$

where $\operatorname{curv}^{2}(\tau)$ denotes the Euclidean curvature of the curve $f(\gamma) \subset \mathbb{R}^{q}$ at $f(x) \in \mathbb{R}^{q}$ for a geodesic $\gamma \subset X$ tangent to $\tau$, and $\|\tau\|$ stands for the Euclidean norm of the vector $D f(\tau) \in T_{f(x)}\left(\mathbb{R}^{q}\right)$.

Observe that this $K^{+}(\tau)$ is a positive polynomial function of degree four on the tangent spaces $T_{x}(X)$ that can be regarded as a 4 -form, that is a symmetric differential form of degree four on $X$.

Let $X=(X . g)$ be a $C^{\infty}$-smooth Riemannian $n$-manifold. Then there exists a continuous 4 -form $K_{0}^{+}$on $X$ that depends on $g$, such that

all continuous 4-forms $K^{+}$on $X$ that are greater than $K_{0}^{+}$, i.e., such that $K(\tau) \geq$ $K_{0}(\tau), \tau \in T(X)$, are realisable as extrinsic curvatures of isometric $C^{2}$-immersions $X \rightarrow R^{q}$ for $q=s_{n}+3 n+5=\frac{(n+2)(n+5)}{2}$. of

Observe that such immersions, which are given by $q$ functions, satisfy a system

$$
q^{+}=\frac{n(n+1)}{2}+\frac{n(n+1)(n+2)(n+3)}{24}
$$

differential equations in $q$ variables, where this system is grossly overdetermined for large $n$.

The simplest instance of such a system can be seen for $X=\mathbb{R}^{n}$, where "largeness of $K^{+}$" reduces to "strict positivity" where a symmetric 4 -form on a linear space $T$ is called strictly positive if the corresponding quadratic form on the symmetric square of $T$ is positive definite 9

Namely let $\left\{K_{i, j, k, l}(x)\right\}, x \in \mathbb{R}^{n}, 1 \leq i, j, k, l \leq n$, be an $n^{4}$-tuple of continuous functions that is symmetric under the permutations of the four indices and such that the corresponding symmetric form $K_{x}^{+}$is strictly positive for all $x \in X$. Then

\footnotetext{
${ }^{9}$ The extrinsic curvatures $K_{+}$of immersions $f: X \rightarrow \mathbb{R}^{q}$ are positive; such $K^{+}$are strictly positive for free immersions $f$, i.e., such that the vectors of the first and second partial derivatives, $\partial_{i} f(x), \partial_{i j} f(x) \in T_{f(x)}\left(\mathbb{R}^{q}\right)$, are linearly independent.
} 
there exists an isometric $C^{2}$-immersion $f: \mathbb{R}^{n} \rightarrow \mathbb{R}^{q}, q=s_{n}+3 n+5=\frac{(n+2)(n+5)}{2}$, such that the second partial derivatives of $f$ satisfy

$$
\sum_{\operatorname{sym}_{i j k l}}\left\langle\partial_{i j} f, \partial_{k l} f\right\rangle=K_{i, j, k, l} 1 \leq j \leq k \leq l \leq n,
$$

where the summation is taken over the 24 permutations of the indices $i, j, k, l 10$

ImPossible Question. Do all sufficiently smooth Riemannian manifolds $X$ admit isometric $C^{2}$-immersions $f: X \rightarrow \mathbb{R}^{q}$ with prescribed curvatures for $q<s_{n}=\frac{n(n+1)}{2}: 11$

Rigidity Problem. The study of "bending" of an $X=X^{n} \subset \mathbb{R}^{q}$, which are non-trivia 12 deformations that preserve the induced Riemannian metrics in $X$, goes back to Gauss and Cauchy. This preceded the very idea of an isometric immersion of an "abstract" manifold $(X, g)$ to $\mathbb{R}^{q}$, but the following issue has remained unsettled even in the local analytic category.

Question. Under what assumptions on $n, q$ and $r$ do there exist a $C^{r}$-smooth and $C^{r}$-rigid (i.e., unbendable) $n$-dimensional submanifold $X$ in the $q$-dimensional Euclidean space $\mathbb{R}^{q}$.

Here, counting parameters tells you that if $q<s_{n}=\frac{n(n+1)}{2}$ and $r$ is large, say $r>\frac{n^{3}}{2}$, then

generic $X^{n} \subset \mathbb{R}^{q}$ must be, locally as well as globally, rigid UNLESS there is some miraculous identity between high derivatives of the (extrinsic) curvatures of this $X^{n}$.

On the other hand, generic $C^{a n}$-submanifolds $X=X^{n} \subset \mathbb{R}^{q}$ are never locally rigid for $q \geq s_{n}$ by the Janet (Burstin) Extension Lemma, which allows extension of isometric $C^{a n}$-immersions $f_{0}$ and their bendings from submanifolds $X_{0} \subset X$ to $X$ under a certain Janet-Burstin genericity/regularity condition on immersions $f_{0}: X_{0} \rightarrow \mathbb{R}^{q}$.

But it is conceivable that

some exceptional "non-Janet" $C^{a n}$-submanifolds $X^{n} \subset \mathbb{R}^{q}$ for $q=s_{n}$

(for $q>s_{n}$ ?) are locally rigid or, at least have "abnormally few" bendings.

(It is even less clear what to expect in the $C^{\infty}$-case.)

In fact this does happen for $n=2$ and $q=s_{2}=3$ by the following amazing

Hopf-Schilt-Efimov Local Rigidity Theorem. The space of germs of analytic surfaces $X \subset \mathbb{R}^{3}$ localised at a point $x_{0} \subset X$ contains a finite codimensional subspace of $C^{\text {an }}$-rigid ones.

The essential feature (Hopf-Schilt, 1939) of these rigid $X \subset \mathbb{R}^{3}$ is that their Gauss maps $G: X \rightarrow S^{2}$ are local ramified coverings of degrees $\operatorname{deg}\left(G_{X}\right)>1$ at $G\left(x_{0}\right) \in S^{2}$ where the $C^{a n}$-rigidity of generic $X$ with $\operatorname{deg}\left(G_{X}\right)=d$ is established (Efimov 1949) for $d=8$. (See 3.1.9 in [35] and references therein.)

Probably, rigidity persists for all $d=3,4, \ldots$, but it is unknown if there are locally $C^{\infty}$-rigid surfaces in the 3 -space (which seems unlikely) and/or if there are

\footnotetext{
${ }^{10}$ This is a correction of the example following theorem (A) in 3.1.5 in 35].

${ }^{11}$ Since such an $f$ cannot be free, one may need to impose some additional "degeneracy condition" on $K^{+}$realisable by curvatures of maps $f$ or to relax the condition on $f$ by prescribing not the full curvature of $f$ but only some function(s) of the curvature.

12 "Trivial" here means coming from isometries of $\mathbb{R}^{q}$.
} 
locally $C^{a n}$-rigid surfaces in $\mathbb{R}^{3}$ with different local topologies of their Gauss maps. And it is hard to imagine $C^{a n}$-rigid surfaces in $\mathbb{R}^{q}$ for $q \geq 4$.

\section{Where Is THE NeW LAND?}

One may continue with questions about relations between regularity classes and the topology/geometry of the source manifolds with the dimensions $q$ of the ambient spaces, but the most compelling problems raised by Nash's results are not about these.

Nash, like Columbus, unintentionally discovered a new land. Refining and improving Nash's isometric imbedding results would be like building bigger and faster ships than those in which Columbus had crossed the Atlantic.

But what is this new land? What is its geography, geology, ecology? How can one explore and cultivate this land? What can one build on this land? What is its future?

It may be hard to decide what this land is, but it is easy to say what it is not: what Nash discovered is not any part of Riemannian geometry, neither does it have much (if anything at all) to do with classical PDEs.

Nash's theorems are only superficially similar to the existence (and non-existence) results for isometric embeddings that rely on PDEs and/or on

relations between intrinsic, i.e., induced Riemannian, and

extrinsic geometries of submanifolds in Euclidean spaces.

(The primary instance of the latter is the proof of the existence of isometric immersions of surfaces with positive curvatures to the Euclidean 3 -space $\mathbb{R}^{3}$ by means of elliptic a priori estimates, that are certain bounds on the extrinsic curvature of a locally convex surface $X \subset \mathbb{R}^{3}$ in terms of the intrinsic Gauss curvature of $X$.)

Nash's results point in the opposite direction:

typically, the geometry of a Riemannian manifold $X$

has no significant influence on its isometric embeddings to $\mathbb{R}^{q}$.

In order to get an idea of what kind of mathematics may lie in this "opposite direction", we shall look at Nash's theorems and his proofs from a variety of different perspectives.

2.1. Waring Connection. "Isometric" for a $C^{1}$-map $f=\left(f_{1}, f_{2}, \ldots, f_{q}\right):(X, g) \rightarrow$ $\mathbb{R}^{q}$ can be written in terms of the differentials of the functions $f_{j}$ as

$$
\sum_{j=1}^{q} d f_{j}^{2}=g
$$

(Here and below $d f^{p}$ stands for $(d f)^{p}$.)

By extending this to $p>2$, we formulate the following

13 Differential Waring Problem 13 What is the minimal $q$ such that all symmetric differential forms $g$ of degree $p=2,3,4, \ldots$ from a given "natural class" of forms on a smooth manifold $X$ admit decompositions to the sums of $p$ th powers of differentials of $C^{r}$-functions $f_{i}: X \rightarrow \mathbb{R}, j=1,2, \ldots, q$ ?

Almost all questions concerning isometric immersions of Riemannian manifolds to Euclidean spaces have their obviously formulated counterparts for $p>2$. But available answers are less satisfactory mainly because of algebraic complications,

\footnotetext{
${ }^{13}$ The classical Diophantine Waring Problem is about decomposing integers to sums of $p$ th powers.
} 
e.g., due to various kinds of "positivity" for forms of even degrees. (Fully working out these algebraic problems may be difficult, but achieving significant progress seems feasible.)

The role of $s_{n}=s_{n, 2}=\frac{n(n+1)}{2}$ is taken here by

$$
s_{n, p}=\frac{n(n+1) \cdots(n+p-1)}{p !}=\frac{n^{p}}{p !}+O\left(n^{p-1}\right), n=\operatorname{dim}(X),
$$

that is the dimension of $p$ th symmetric power of the linear $n$-space.

Thus, the representation $\sum_{i=1}^{q} d f_{i}^{p}=g$ amounts to a system of $s_{n, p}$ partial differential equations of the first order imposed on the functions $f_{i}$ on $X, i=1, \ldots, q$; hence, the $C^{\infty}$-solution for general $g$ is expected only for $q \geq s_{n, p}$.

But the $q=q(n, p)$ available today for $p>2$ are much bigger than $s_{n, p}$.

For instance, Nash's $C^{r}$-perturbation techniques, combined for even $p \geq 4$ with Hilbert's decomposition (see below), show 14 that if $r>2$ (including Hölder $C^{r, \alpha}$ for $r+\alpha>2$ as well $r=\infty$, an), then

( $C^{r}$-forms $g$ on (possibly non-compact) $n$-manifolds $X$ are decomposable to sums of $p$ th powers of differentials of $C^{r}$-functions $f_{i}$ on $X$,

$$
g=\sum_{i=1}^{q} d f_{i}^{p}
$$

for

$$
q=(2 p+1) s_{n, p}+\operatorname{const}_{p} \frac{s_{n}}{n},
$$

where the forms $g$ for even $p$ need to be positive definite when regarded as quadratic forms on the symmetric square $T(X)^{\frac{p}{2}}$ of the tangent bundle of $X$.

Turning to $C^{1}$-decompositions for continuous forms $g$ to sums $g=\sum_{i=1}^{q} d f_{i}^{p}$, observe that the natural lower bound on $q$ for $p>2$ is

$$
\underline{q}=\underline{q}_{n p}=s_{n p} / n
$$

as explained below. (It is $\approx \frac{2 s_{n, 2}}{n}$ for $p=2$ due to the symmetries of quadratic forms.)

But the known upper bounds on $q$ remain poor:

$(\star \star) q=2 p \underline{q}_{n p}$ for odd $p$ and something of order $4 p s_{n p}=4 p n \underline{q}_{n p}$ for (positive definite) forms on $X$ of even degrees $p$.

Polynomial Waring Problem. A decomposition $g=\sum_{i=1}^{q} d f_{i}^{p}$, when restricted to a tangent space of $X$ at a point $x \in X$, amounts to a representation of the algebraic form $g_{x}$, which is a homogeneous polynomial of degree $p$ on the tangent space $T_{x}(X)$, as the sum of $p$ th powers of linear forms.

The topology of the spaces of such representations, in particular what is needed for the analysis of decompositions $g=\sum_{i} d f_{i}^{p}$, is more complicated for $p>2$ than for $p=2$.

One does not seem to know if the minimal $q$ needed for the decomposition

$$
\sigma\left(x_{i}\right)=\sum_{i=1}^{q} l_{j}\left(x_{i}\right)^{p}
$$

\footnotetext{
${ }^{14}$ See 10.2 .3 in 34 and 3.1.4 in 35.
} 
for an arbitrary form (i.e., homogeneous polynomial) $\sigma$ of degree $p$ in $n$ variables $x_{i}$ is comparable with $\underline{q}_{n, p}=s_{n, p} / n$, which is the lower bound for such decompositions of generic $\sigma$.

(The space $\Sigma_{n, p}$ of our forms $\sigma$ has dimension $s_{n, p}$, while the subset $\Sigma_{q} \subset \Sigma_{n, p}$ of forms induced from $\sum_{1}^{q} y_{j}^{p}$ by linear maps $\mathbb{R}^{n} \rightarrow \mathbb{R}^{q}$ has dimension $\leq n q$; hence, $\Sigma_{q}$ is nowhere dense in $\Sigma_{n, p}$ for $q<\underline{q}_{n, p}=s_{n, p} / n$.)

What is obvious however (Carathéodory theorem), is the existence of the decomposition $\sigma\left(x_{i}\right)=\sum_{i=1}^{q} l_{j}\left(x_{i}\right)^{p}$ with $q=s_{n, p}$ for the forms $\sigma$ that are contained in the convex cone $C_{n, p} \subset \Sigma_{n, p}$ spanned by the forms $l\left(x_{i}\right)^{p}$.

(If $p$ is even, then, obviously, $\Sigma_{n, p}$ contains exactly two minimal non-zero closed convex cones invariant under the action of the linear group $G L(n)$, that are $C_{n, p}$ and $-C_{n, p}$. But if $p$ is odd, the only non-zero convex $G L(n)$-invariant cone is $\Sigma_{n, p}$ itself.)

Example: Hilbert's decomposition. Let $Q=Q\left(x_{i}\right)$ be a positive definite quadratic form in variables $x_{1}, \ldots, x_{i}, \ldots, x_{n}$, e.g., $Q\left(x_{i}\right)=x_{1}^{2}+\cdots+x_{i}^{2}+\cdots+x_{n}^{2}$. Then, for all even $p=2 k$, there exist linear forms $l_{j}=l_{j}\left(x_{i}\right), j=1,2, \ldots, q \leq s_{n, p}$, such that

$$
Q^{k}=\sum_{j=1}^{q} l_{j}^{p}
$$

Proof. The action of the orthogonal group $O_{Q} \subset G L(n)$ (of linear $Q$-isometries of $\mathbb{R}^{n}$ ) on $\Sigma_{n, p}$ has a unique invariant line, say $L_{Q} \subset \Sigma_{n, p}$; hence $L_{Q}$ must be equal $\left\{\lambda Q^{k}\right\},-\infty<\lambda<\infty$.

It follows that the barycenters of the orbits $o\left(l^{p}\right), o \in O_{Q}$, equal $\lambda Q^{p}$, for $\lambda=$ $\lambda(l)>0$, for all non-zero linear forms $l$; thus, $Q^{k}$ is contained in the conical convex hull of each such orbit. QED

(Since, clearly, $Q^{k}$ is contained in the interior of the convex hull of the forms $l^{p}$, obvious linear algebra shows that if the form $Q$ is rational (i.e., with rational coefficients), then the decomposition $Q^{k}=\sum_{j} \alpha_{i} l_{j}^{p}$ can be taken with rational numbers $\alpha_{j}$ and forms $l_{j}$ as well. This was used by Hilbert in his reduction of the Waring problem from $2 k$ to $k$.)

\section{QUESTIONS}

What is the minimal $q$ needed for a representation $Q^{k}=\sum_{i}^{q} l^{p}$ ?

This may need a sharpening of the Carathéodory theorem to convex sets with "large", in particular of dimensions $n>0$ (and symmetric? "well spread" on the boundary of the convex set?), sets of extreme points.

What are, in general, homotopy properties of the space $F_{q}(\sigma) \subset \operatorname{Hom}\left(\mathbb{R}^{n}, \mathbb{R}^{q}\right)$ of representations $\sigma=\sum_{i}^{q} l^{p}$ for a given form $\sigma$ and of the non-singular locus in $F_{q}(\sigma)$ ?

Essential results here with an emphasis on rationality, e.g., Birch's theorem, can be found in Davenport's book (1962) [22], and the geometry of these representations is discussed in 2.4.9 and 3.1.4 in [35] and is advanced further by Dolnikov and Karasev (2011) 25] in the ambience of Dvoretzky-type theorems.

Generalisations. One may replace functions $f_{j}$ on $X$ in $g=\sum_{j} d f_{j}^{p}$ by differential forms of degree $m>0$, with $g$ and $d f_{j}^{p}$ belonging to the $p$ th symmetric power of the bundle $\Lambda^{m+1}(X)$ of the exterior $(m+1)$-forms on $X$. 
And one may impose additional constraints on such representations of $g$ by requiring the forms $d f_{j}$ to be exterior products of exact forms $\phi_{j, \nu}$ of degrees $m_{\nu}$ with $\sum_{\nu} m_{\nu}=m+1$, e.g., where all $m_{\nu}=1$, where the unknown become functions $\psi_{j, 1}, \ldots, \psi_{j, k}, \ldots, \psi_{j, m}$ on $X$ for the equation

$$
\sum_{j}\left(\bigwedge_{k} d \psi_{j, k}\right)^{p}=g
$$

Also, one may use non-linear differential operators between spaces of tensors instead of the exterior differential $d$, e.g., the assignment of the curvature tensors to positive definite quadratic differential forms $g$ on $X$, say $g \mapsto K(G)$, where one looks for decompositions of an arbitrary tensor $R$ of the curvature type to the sum $R=\sum_{j} K\left(g_{j}\right)$.

Probably, general Nash-style methods from [35. can be applied here, but they are unlikely to deliver the conjectural optimal solvability results.

\section{Similarity and Dissimilarity of Geometry with Arithmetic}

Is drawing parallels between differential and Diophantine decomposition problems justifiable?

The algebraic problems arising in both cases are rather similar, where, in the differential case, algebra must be augmented by algebraic topology that may also be present in the arithmetic category. Also the "analytic logic" of the Nash perturbation techniques used for differential equations has something in common with the circle method - the basic technical tool in this kind of Diophantine analysis. But there are no (?) apparent number theoretic counterparts to the Nash-style direct soft geometric constructions of solutions of PDEs.

The dream of bringing the two theories to a common ground may never come true, but transporting formulations of problems from one domain to another may bring along something new and interesting, e.g., the tensorial nature of the differential Waring problem suggests looking at the Waring problem in the semiring of matrices with positive integer entries.

\subsection{Infinite Dimensional Representations of Diff, Invariant Convex Cones} and Permutations of Primes. Riemannian metrics $g$ on a smooth manifold $X$ that can be induced by $C^{r}$-maps from $X$ to Euclidean spaces constitute a convex cone, call it Cone $_{\text {Euc }}^{r}=\operatorname{Cone}_{\text {Euc }}^{r}(X)$ in the linear space of all quadratic forms on $X$.

Nash's existence theorem for smooth embeddings $X \rightarrow \mathbb{R}^{q}$ (the proof of which, as given by Nash, relies on the addition of metrics in this cone) says, in effect, if you are indifferent to a bound on the dimension $q$ of the Euclidean space where $X$ goes, that

Cone $_{\mathrm{Euc}}^{r}$, at least for $r=\infty$, coincides with the cone of all positive definite $C^{r}$-forms on $X$.

(This is also true for $r=a n$; if $2<r<\infty$, then Nash's theorem says that Cone Euc is slightly larger than the cone of all $C^{r}$-forms.)

Let us formulate a version of this theorem by Nash for a rather general class of cones, namely to those that are invariant under the natural action of the group $\operatorname{Diff}^{r+1}=\operatorname{Diff}^{r+1}(X)$ of $C^{r+1}$-diffeomorphisms of $X$ on quadratic differential $C^{r}$ forms on $X$ (compare 3.1.3 in [35]). 
If $X$ is a compact $C^{\infty}$-manifold, then there is no non-trivial Diff ${ }^{\infty}$-invariant cone in the space of quadratic $C^{\infty}$-forms on $X$ : every non-zero cone (e.g., Cone $_{\text {Euc }}^{\infty}$ ) must contain all quadratic $C^{\infty}$-forms on $X$.

Similarly, there are no non-trivial Diff ${ }^{a n}$-invariant cones in the space of quadratic $C^{a n}$-forms on compact manifolds $X$ and/or no Diff ${ }^{1}$-invariant cones in the space of continuous forms, but it is unclear what happens in the $C^{r}$-case for $2 \leq r<\infty$.

On the other hand, if $X$ is non-compact, there are hordes of invariant cones associated with monotone geometric invariants of $X$ at infinity, say $\operatorname{inv}_{\infty}(X)$, where the cones are defined by $\operatorname{inv}_{\infty}(X)=\infty$. Thus, complete metrics, metrics with infinite volumes and metrics with infinite diameters at infinity, metric with infinite $k$-widths or $k$-waists at infinity, etc., make such cones.

Can one enlist convex Diff-invariant cones of Riemannian metrics on noncompact manifolds?

The study of these cones belongs with what is called metric geometry which is far removed from "Nash's land", while the relevant questions for the moment are the following.

A. What are "natural" linear spaces acted upon by $\operatorname{Diff}=\operatorname{Diff}^{\infty}(X)$ ?

B. What are invariant linear subspaces in these spaces?

C. What are invariant convex cones in there?

D. What are other groups, semigroups, categories, Lie algebras, besides Diff where $\boldsymbol{A}, \boldsymbol{B}$, and $\boldsymbol{C}$ make sense?

We shall address these questions later in this article, but we mention here that there is an arithmetic counterpart to this "conical philosophy" where the role of $\operatorname{Diff}(X)$ acting on $X$ is played by the permutation group $\Pi=\Pi(\mathcal{P}(\mathbb{N}))$ of the set $\mathcal{P}$ of prime numbers and where this $\Pi$ acts on the set $\mathbb{N}$ of positive integers.

One knows, concerning this action, that

if a non-empty subset $M \subset \mathbb{N}$ is invariant under $\Pi$, then there are numbers $q=q(M)$ and $k=k(M)$, such that every positive integer $n \geq k$ decomposes to the sum of at most $q$ numbers $m_{i}=m_{i}(n) \in M$.

Example. Given $d=1,2,3, \ldots$, every sufficiently large integer $n \geq k=k(d)$ is the sum of at most $q=q(d)$ of $d$ th powers of prime numbers.

\subsection{Maps Between Manifolds, Induced Structures, Composability, and}

Functoriality. An essential feature of isometric maps between Riemannian manifolds is that composition of isometric immersions (embeddings) $X \rightarrow Y$ and $Y \rightarrow Z$ is an isometric immersion (embedding) $X \rightarrow Z$.

However simple, this property is quite uncommon for solutions of classical PDEs (with the notable exception of the Cauchy-Riemann equations).

The analytic manifestation of composability is that the systems of isometric immersion equations are degenerate: an isometric map $f:(X, g) \rightarrow(Y, h)$, that is subject to the equation $f^{\star}(h)=g$, cannot be assumed arbitrary on any hypersuface $X_{0} \subset X$, since there it must satisfy the equation $f_{\mid X_{0}}^{\star}(h)=g_{\mid X_{0}}$.

On the other hand, composability allows one to formulate the concept of an isometric immersion in functorial terms without direct reference to PDEs as follows.

In fact, $C^{r}$-Riemannian manifolds $X$ can be represented by covariant functors from the category $\mathcal{I}$ of intervals $I \subset \mathbb{R}$ and translations $I_{1} \rightarrow I_{2}$ to the category of topological spaces. Namely, a manifold $X$ is represented by the totality of the 
spaces of isometric $C^{r}$-immersions $I \rightarrow X, I \in \mathcal{I}$, say $\mathcal{F}_{X}$, and isometric immersions $X \rightarrow Y$ correspond to natural transformations of the functors $\mathcal{F}_{X} \sim \mathcal{F}_{Y}$.

Most problems concerning isometric immersions $X \rightarrow \mathbb{R}^{q}$ are more naturally formulated for general Riemannian manifolds instead of the Euclidean spaces $\mathbb{R}^{q}$, and many proofs work in this setting with obvious adjustments where they gain extra power.

Thus, for instance,

the $C^{1}$-theory effortlessly extends to isometric $C^{1}$-immersions $X \rightarrow Y$ between arbitrary manifolds with continuous Riemannian metrics, provided $\operatorname{dim} Y>\operatorname{dim} X$. This, in full generality, was proven by Nico Kuiper, who, in 1955, extended Nash's 1954 theorem to the case $\operatorname{dim}(Y)=\operatorname{dim}(X)+1$.

Although Nash's proof of his $C^{\infty}$-immersion theorem $X \rightarrow \mathbb{R}^{q}$ does not directly apply to immersions $X \rightarrow Y$, the $C^{\infty}$-analytic techniques developed by Nash can be combined with the geometric idea behind his $C^{1}$-construction.

This allows

$C^{\infty}$ - and $C^{a n}$-immersions $X^{n} \rightarrow Y^{q}$ starting from $q=s_{n}+2 n+3, s_{n}=\frac{n(n+1)}{2}$, according to 35] and, significantly better, for all $q \geq s_{n}+n+5$ according to Günther.

However, the situation with $C^{r}$-immersions for $2 \leq r<\infty$ remains unsatisfactory, since Nash's analytic perturbation techniques require excessive regularity assumptions on $Y$. (Possibly, these assumption can be relaxed with Günther's modification of Nash's proof of the perturbation theorem.)

Now, as in $\mathbf{A}$ from the previous section (see p. 184), we ask ourselves

What is the maximal natural class $\mathcal{G}_{\text {funct }}$ of (differential?) geometric structures with similar composability/functoriality properties?

In some respects $\mathcal{G}_{\text {funct }}$ is more restricted than the class $\mathcal{G}_{\text {inv }}$ which makes Diffinvariant cones.

For instance, $\mathcal{G}_{\text {inv }}$ contains all kind of tensors, while, as far as tensors are concerned, the functoriality of $\mathcal{G}_{\text {funct }}$ allows only contravariant ones - differential forms of all degrees and all kinds of symmetries but nothing, say, as simple as (covariant) vector fields.

On the other hand, $\mathcal{G}_{\text {funct }}$ requires no additive structure of their members, thus, for instance, allowing subbundles of the tangent bundle, e.g., contact structures.

First order structures, such as tensors and contact-like structures, are defined via linear maps between tangent spaces of manifolds, where the maps of interest are differentials (first derivatives) of smooth maps. With this is mind we make the following

Definitions. Let $\mathcal{F}_{r}$ be the category where objects are germs of smooth manifolds $X$ at points $x \in X$ with morphisms $(X, x) \rightarrow(Y, y)$ being $r$-jets, $r=$ $0,1,2,3, \ldots$, of smooth maps $(X, x) \rightarrow(Y, y)$ (of course, one does not need here manifolds and smooth maps themselves but rather their "formal shadows").

$A$ (contravariant) $r$ th order geometric structure type $[g]$ is a contravariant functor from $\mathcal{F}_{r}$ to the category of real algebraic varieties and regular morphisms. (To feel comfortable, one must restrict all this to some small subcategory of $\mathcal{F}$.)

Such a type $[g]$ naturally defines a fibration, call it $[G]$ over $X$, for all smooth manifolds $X$, where sections $g$ of these bundles are called structures of type $[g]$ and where $C^{r}$-smooth maps $f: X \rightarrow Y$ send such structures from $Y$ to $X$, denoted 
$h \stackrel{\leftrightarrow}{f} g=\left(J_{f}^{(r)}\right)^{\star}(h)$, where $J_{f}^{(r)}$ stands for the $r$ th order jet of $f$ which incorporates all partial derivatives of $f$ up to order $r$,

Observe, that $f \mapsto g=\left(J_{f}^{(r)}\right)^{\star}(h)$ is a (non-linear in most cases) differential operator of order $r$.

Subcategories of $\mathcal{F}_{r}$. This definition also makes sense for various subcategories $\mathcal{E}$ of $\mathcal{F}_{r}$.

For instance if one takes $\mathcal{E} \subset \mathcal{F}_{r}$ which consists of the germs $(X, x)$ of a single manifold $X$ for all $x \in X$, such that the morphisms in $\mathcal{F}_{r}$ come from local diffeomorphisms $\left(X, x_{1}\right) \rightarrow\left(X, x_{2}\right)$ and the type-defining functor takes values in the category of vector spaces, then one arrives at a vector bundle naturally acted upon by $\operatorname{Diff}(X)$, and the space of sections is where one may start speaking of invariant cones as in the previous section.

Other interesting categories $\mathcal{E}$ (we shall return to them later on) are

- category of symplectic (contact) manifolds and symplectic (contact) maps between them;

- category of complex analytic manifolds;

- category of (smooth?) algebraic varieties over a field $\mathbb{F}$ of characteristic zero (e.g., $\mathbb{Q}, \mathbb{R}$ or $\mathbb{C}$ ) and regular maps.

EXAMPLES. One may wonder if higher order structures ever come about in geometry and if the general categorical setting is needed to encompass geometrically attractive examples.

In fact one of the first theorems one encounters in differential geometry is that of the existence of space curves with given curvature and torsion which are second and, respectively, third order structures (see section 4.2).

These generalise to manifolds of dimensions $n>1$ where they satisfy counterparts to Nash's embedding theorem, which allows us, in particular, to construct isometric immersions with prescribed external curvatures.

And thinking categorically opens a road to the study of many similar geometric phenomena.

19 Question. Do higher order "isometric embedding" problems have Diophantine counterparts, e.g., along the lines of the Waring problem?

2.4. Homotopy Theoretic Perspective on PDE and PDR. An emphasis on the existence theorems for (systems of partial) differential equations

$$
\mathcal{D}(f)=g
$$

is justified in so far as their solutions are unique under certain well-shaped (e.g., initial or boundary) conditions or at least if the spaces of solutions are rigidly structurally organised.

But the solutions of the isometric immersion equations

$$
\mathcal{D}_{h}(f)=(D f)^{\star}(h)=g
$$

for maps between Riemannian manifolds, $f:(X, g) \rightarrow(Y, h)$, that are delivered by Nash's theorems and their generalisations are nothing of the kind: the spaces of these solutions are vast and, from the analytic point of view, amorphous; being onto reflects only a tiny part of properties of the operator $\mathcal{D}_{h}$. 
A more pertinent question concerning differential operators $\mathcal{D}=\mathcal{D}_{h}: F \rightarrow G$ that represent inducing geometric structures $g$ on $X$ (e.g., Riemannian metrics) from $h$ on $Y$ by smooth maps $f: X \rightarrow Y=(Y, h)$ is not if they are onto, but as follows.

What is the maximal domain $F_{\text {reg }} \subset F$ such that the restriction of $\mathcal{D}$ to $F_{\text {reg }}$ is a Serre fibration $F_{\text {reg }} \rightarrow G$ ?

This question for isometric $C^{1}$-immersions was fully resolved by Nash's proof (1954) 62 of his $C^{1}$-theorem that was extended by Nicolas Kuiper (1955) [53] to the case of immersions and embeddings $X^{n} \rightarrow \mathbb{R}^{q}$ for $q=n+1$ that was left open by Nash. In fact, the Nash-Kuiper argument delivers the following

$C^{1}$-Fibration Theorem. Let $F$ be the space of $C^{1}$-immersions or embeddings from a smooth $n$-manifold $X$ to a Riemannian manifold $Y=(Y, h)$, and let $G$ denote the space of continuous Riemannian metrics on $X$.

If $Y=(Y, h)$ is an $n$-dimensional Riemannian manifold such that the balls around some point $y_{0} \in Y$ are convex, e.g., $(Y, h)=\left(\mathbb{R}^{q}, \sum_{1}^{q} d y_{i}^{2}\right)$, if $q \geq n+1$ and if $X$ is compact, then the map $\mathcal{D}_{h}: F \rightarrow G$ is a Serre fibration.

Moreover, with no restriction on $Y$ and $X$, the map $\mathcal{D}_{h}$ satisfies Serre's homotopy lifting property for "increasing paths" $g_{t}$ in $G$, i.e., paths such that $g_{t_{2}}-g_{t_{1}}$ is positive definite for all $t_{2}>t_{1}$.

This implies, for instance, the existence of $C^{1}$-embeddings $\left(X^{n}, g\right) \rightarrow \mathbb{R}^{2 n}$ for all $g$ since a Serre fibration of a non-empty space $F$ over a path connected $G$ is necessarily (and obviously) onto.

The original Nash construction of isometric $C^{\infty}$-embeddings did not yield a similar fibration theorem, but this can be achieved by combining the Nash $C^{1}$ construction with his $C^{\infty}$-techniques:

$C^{\infty}$-Fibration Theorem. The map $\mathcal{D}_{h}$ from the $\left(\right.$ sub) space $F_{\text {free }}^{\infty} \subset F$ of fre 15 $C^{\infty}$-immersions (or embeddings) $X \rightarrow Y$ to the space $G^{\infty}$ of smooth metrics on $X$ is a Serre fibration, provided $X$ is compact and $Y=(Y, h)$ is a $C^{\infty}$-smooth manifold of dimension $q=\operatorname{dim}(Y) \geq s_{n}+2 n+3, s_{n}=\frac{n(n+1)}{2}$, that satisfies the above convexity property, where compactness of $X$ and convexity of $Y$ are unneeded for Serre's homotopy lifting property for "increasing paths" $g_{t}$ in $G^{\infty}$.

This, in the $C^{\infty}$ as well as in the $C^{a n}$-case, follows from the construction of isometric immersions by adding $d \phi^{2}$ (see section 3.12).

CONJeCture. The $C^{\infty}$-fibration property holds for $q \geq s_{n}+n+116$

On the other hand, the dimension $q=s_{n}+n$-that is the minimal $q$ that, locally, allows free maps $X^{n} \rightarrow Y^{q}$ - seems beyond reach. For instance the following question raised about half a century ago remains unanswered:

Do the $n$-tori admit free (forget isometric!) immersions to $\mathbb{R}^{s_{n}+n}$ for $n \geq 2$ ?

But freedom is not indispensable for smooth isometric immersions. Probably,

there are subsets $F_{\text {reg }} \supset F_{\text {free }}$ in the spaces of smooth maps $f: X \rightarrow Y$ that do fibre over $G^{\infty}$, for smaller $q$, possibly up to $q=s_{n}+1$.

\footnotetext{
${ }^{15}$ A map $f: X \rightarrow(Y, h)$ is free if its first and second covariant partial derivatives in $(Y, h)$ are linearly independent at all points in $X$ or, equivalently, if its (second) osculating spaces everywhere have dimensions $s_{n}+n, n=\operatorname{dim}(X)$. Observe, that if $q=\operatorname{dim} Y \geq s_{n}+2 n$, then generic $C^{\infty}$-maps $X \rightarrow Y$ are free by Thom's transversality theorem.

${ }^{16}$ Those who understand Günter's construction would have no difficulty in proving it for $q \geq s_{n}+n+3$.
} 
Question. If $B$ is a locally/infinitesimally partially ordered space, one can speak of increasing/directed paths in $B$ and of the homotopy lifting property of these paths to $A \rightarrow B$.

Is the class of maps $A \rightarrow B$ that admit such "directed homotopy liftings" worth giving a name?

2.5. Stephen Smale and the $h$-Principle. The idea of using homotopy theory in geometric problems goes back to Stephen Smale's "turning the 2-sphere inside out" (1958) 68.

This was followed by Smale's 1959 paper on immersions of the $n$-spheres [69] that was extended by Morris Hirsch (1959) [45] to general manifolds. This furnished the fully fledged immersion theory, which is what is now-a-days called the $h$-principle for differentiable immersions between smooth manifolds, $X^{n} \rightarrow Y^{q}$ for $q>n$.

Amusingly, the basic geometric construction of "wrinkling", invented by Smale and adopted by Hirsch, was similar to that by Kuiper in the proof of the isometric $C^{1}$-embedding theorem.

In fact, albeit neither Kuiper nor Nash before him were thinking in homotopy theoretic terms, a (simplified) version of the Nash-Kuiper construction allows a half page proof (instead of the original 17 and 34 pages, respectively) of the 1959 immersion theorems of Smale and Hirsch.

On the other hand, the homotopy theoretic logic of Smale's proof of the $h$ principle for the (partial differential) relation $\operatorname{rank} D f=n$, which is a discriminant non-vanishing condition that characterises immersions $f: X \rightarrow Y$, is also central in the study of the (partial differential) equations that define structure inducing maps, including isometric immersions $X^{n} \rightarrow \mathbb{R}^{q}$.

Below is an example of an attractively simple existence/approximation theorem, where the logic of the proof (by convex integration) is rooted in homotopy theory.

Euclidean Short Approximation Theorem. Let $X$ be a smooth $n$-dimensional manifold that admits a smooth immersion to $\mathbb{R}^{q}$, let $g$ be a Riemannian metric on $X$, and let $f_{0}:(X, g) \rightarrow \mathbb{R}^{q}$ be a curve shortening map:

$$
\text { length }_{\mathbb{R}^{q}}\left(f_{0}(C)\right)<\operatorname{length}_{g}(C) \text { for all smooth curves } C \subset X \text {. }
$$

Then, provided $q>n$, the map $f_{0}$ can be uniformly approximated by isometric $C^{1}$-immersions $(X, g) \rightarrow \mathbb{R}^{q}$.

The novelty here is the approximation of $f_{0}$ by curve shortening immersions which does not follow either from Smale-Hirsch theory, which allows an approximation of $f_{0}$ by smooth immersions $X \rightarrow \mathbb{R}^{q}$ with no shortness condition, nor by the Nash-Kuiper construction, which needs a curve shortening immersion (or embedding) as a starting point.

What goes to the proof of this short approximation theorem is convex integration - a common descendent of the ideas and methods that originated in the works by Smale and by Nash (see 2.4 in [35, and [70, 27]).

\section{Basic Constructions}

The classical proofs of the existence theorems for geometric PDEs commonly rely on a priori estimates that quantify the uniqueness properties of the solutions.

This approach fails for most equations characterising maps $f$ that induce prescribed geometric structures, where the solutions show no trace of uniqueness and 
where the success in the case of isometric immersions was achieved by Nash by forfeiting classical PDEs and inventing several direct constructions of such maps.

In the following sections we shall present variants of these constructions which are of interest in their own right regardless of their current roles in the solution of the isometric immersion problem.

\subsection{Kuratowski-Weyl Embeddings and Nash Spherical Decomposition.}

Given a function in one positive variable $d \mapsto \phi(d)$, define the map from a metric space $X$ to the space of functions $\psi(x)$ on $X$ by $x^{\prime} \mapsto \phi\left(\operatorname{dist}_{X}\left(x^{\prime}, x\right)\right)$ for all $x^{\prime} \in X$, denoted

$$
K W_{\phi}: X \rightarrow \mathbb{R}^{X}
$$

If $\phi(r)=r$, then this map is distance preserving for the space of functions on $X$ with the sup-norm.

But we are more interested at the moment with the Hilbert space $L_{2}(X)$ that is the space of functions on $X$ with the $L_{2}$-norm, where, following Hermann Weyl, we observe that

if $X$ is a complete Riemannian flat manifold and $\phi$ is a square integrable function with the support in $[0, \varepsilon]$ for a small positive $\varepsilon$ (depending on $X$ ), then there exists a unique (normalising) constant $c=c(X, \varepsilon)$, such that the map $K W_{c \phi}: X \rightarrow L_{2}(X)$ is an isometric immersion.

In general, if $X$ is an arbitrary compact Riemannian manifold $(X, g)$ and $\phi_{\varepsilon}$ is the characteristic function of the interval $[0, \varepsilon]$, then there are constants $c_{\varepsilon}$ such that

the Riemannian metrics $g_{\varepsilon}$ induced by the maps $K W_{c_{\varepsilon} \phi_{\varepsilon}}: X \rightarrow L_{2}(X)$ uniformly converge to $g$ for $\varepsilon \rightarrow 0$.

By making the constants $c_{\varepsilon}$ small, one may assume that the difference $\delta_{\varepsilon}-$ $g-g_{\varepsilon}$, besides being as small as you want, is also positive definite and thus, also approximable by metrics induced by maps to a Hilbert space. fore,

Hence, $g$ is representable by a countable sum of Hilbert-induced metrics. There-

every positive definite quadratic differential form on a compact manifold $g$ decomposes into a convergent series

$$
g=\sum_{i=1}^{\infty} d f_{i}^{2}
$$

which defines an isometric immersion $(X, g) \rightarrow \mathbb{R}^{\infty}$.

Now, let $X$ be a (possibly) non-compact manifold, and let $B_{j} \subset X, j=1,2,3, \ldots$, be smooth compact $n$-balls, such that their interiors furnish a locally finite cover of $X$ (of multiplicity $n+1$ if you wish).

Then, following Nash, let $\sigma_{j}: X \rightarrow S^{n}$ be $C^{\infty}$-smooth maps, which are diffeomorphic on the interiors of the balls and which send their complements to the south pole of the sphere $S^{n}$. It is obvious that

given a Riemannian metric $g$ on $X$, there exist metrics $g_{j}$ on $S^{n}$ such that

$$
\sum_{j}\left(D \sigma_{j}\right)^{\star}\left(g_{j}\right)=g .
$$


This, along with the above decomposition of Riemannian metrics to sums $\sum_{i} d f_{i}^{2}$ on compact manifolds (where the ones we need here are diffeomorphic to $S^{n}$ ) shows that

every Riemannian metric $g$ on a smooth manifold $X$, be it compact or not, decomposes to a convergent sum $\sum_{i} d f_{i}^{2}$ where the functions $f_{i}$ have their supports contained in given balls $B_{i} \subset X$ that cover $X$.

About Smoothness and Convergence. One sees instantaneously that this sum uniformly (i.e., in the $C^{0}$-topology) converges for all continuous metrics $g$; probably, this convergence is (can be forced to be?) $C^{r}$ for $C^{r}$-metrics.

On Weyl's Operator. Given a (preferably) smooth measure $d x$ on a smooth compact manifold $X$, every, say, continuous function in two variables, $k(x, y)$ defines a map from $X$ to the Hilbert space $L_{2}(X)$ by $x \mapsto f_{x}(y)=k(x, y)$ that induces a Riemannian metric on $X$, call it $W(k)$. What of interest may be said about this map $k \mapsto W(k)$ ?

Historical Remark. Nash used such a "spherical decomposition" in his 1956 paper 63 to show that the existence of isometric $C^{\infty}$-immersions of compact $n$-manifolds (diffeomorphic to $S^{n}$ ) to $\mathbb{R}^{q}$ yields such immersions of possibly non-compact $X$ to $\mathbb{R}^{(n+1) q}$. Thus, he proved that all $C^{\infty}$-manifolds of dimension $n$ admit isometric $C^{\infty}$ immersions of $X$ to the Euclidean space of dimension $q_{\text {Nash }}=(n+1)\left(\frac{3 n(n+1)}{2}+4 n\right)=$ $\frac{3 n^{3}}{2}+\frac{11 n^{2}}{2}+\frac{11 n}{2}$.

Later on, in 1969-1970, R. E. Greene 31, 32 and J. S. Clarke 14 modified Nash's decomposition and thus obtained immersions of non-compact $X$ to the Euclidean spaces of dimensions $q_{\text {Greene }}=12 n^{2}+34 n+14$ and $q_{\text {Clarke }}=\frac{n^{3}}{3}+\frac{5 n^{2}}{2}+\frac{37 n}{6}+1$.

Although these bounds on $q$ have been superseded by later constructions of isometric $C^{\infty}$-immersions/embeddings $X^{n} \rightarrow Y^{q}$ non-discriminantly for all $C^{\infty}$ Riemannian manifolds $X^{n}$ and $Y^{q}$ with $q \geq s_{n+2}=\frac{n^{2}}{2}+\frac{5 n}{2}+3$ (even better, for $q \geq s_{n+1}+4=\frac{n^{2}}{2}+\frac{3 n}{2}+5$ if you accept Günter's construction), Nash's spherical decomposition and its variations remain useful for inducing geometric structures, such as higher degree differential forms, where more refined constructions are still unavailable.

Keeping this in mind and following in the steps of Greene and Clarke, we indicate below yet another version of such a decomposition.

Cover a (non-compact) manifold $X$ by three open subsets, where each of these equals the union of disjoint relatively compact subsets, say $U_{j, k} \subset X, j=1,2, \ldots$, $k=1,2,3$, such that the boundaries of all $U_{j, k}$ are smooth and mutually disjoint and, moreover, such that each point $x \in X$ is contained in at least two of $U_{j, k}$. Observe, that

if smooth maps $\sigma_{j, k}: X \rightarrow S^{n}$ that are constant outside $U_{j, k}$ are chosen generically, then every "structure" $g$ on $X$ from a given "additive class" $\mathcal{G}$ can be decomposed as

$$
g=\sum_{j, k}\left(D \sigma_{j, k}\right)^{\star}\left(g_{j, k}\right) \text { for some structures } g_{j, k} \text { on } S^{n} \text { from the class } \mathcal{G} .
$$

Thus,

the existence of "isometric immersions" of the manifolds diffeomorphic to $n$ spheres to $\mathbb{R}^{q}$ implies the existence of such immersions of ALL $n$-manifolds $X$ to $\mathbb{R}^{3 q}$. 
Question. The above "3" can be, obviously, replaced by "2" if $X$ is diffeomorphic to $\mathbb{R}^{n}$. But what is possible for other manifolds?

\subsection{Adding $d \phi^{2}$ to Induced Riemannian Metrics $g$ by $C^{1}$-Twist and the} Proof of Nash's $C^{1}$-Immersion Theorem. Let $f:(X, g) \rightarrow Y=(Y, h)$ be an isometric $C^{r}$-immersion.

EXTENSION $f_{\times 2}$ AND Condition $\left[\times D^{2}(\varepsilon)\right]$. Suppose $f$ admits a $C^{r}$-extension from $X=X \times 0 \subset X \times \mathbb{R}^{2}$ to $X \times \mathbb{R}^{2}$, such that

this extension, call it $f_{\times 2}: X \times \mathbb{R}^{2} \rightarrow Y$, is an isometric immersion for the product metric $g \oplus\left(d r_{1}^{2}+d r_{2}^{2}\right)$ on the $\varepsilon$-neighbourhood $X \times D^{2}(\varepsilon) \subset X \times \mathbb{R}^{2}$ of $X \subset X \times \mathbb{R}^{2}$, where $D^{2}(\varepsilon) \subset \mathbb{R}^{2}$ denotes the $\varepsilon$-disc in the plane $\mathbb{R}^{2}$ around the origin $0 \in \mathbb{R}^{2}$.

$\left[+d \phi^{2}\right]$-Lemma. Assume $\left[\times D^{2}(\varepsilon)\right]$, and let $\phi: X \rightarrow \mathbb{R}$ be a $C^{r}$-function on $X$. Then there exists a $C^{r}$-immersion $f_{\varepsilon}: X \rightarrow Y$, such that

(1) $\operatorname{dist}_{Y}\left(f(x), f_{\varepsilon}(x)\right) \leq \varepsilon$ for all $x \in X$;

(2) the metric induced by $f_{\varepsilon}$ on $X$ satisfies

$$
\left(D f_{\varepsilon}\right)^{\star}(h)=g+d \phi^{2} .
$$

Proof. Let $s_{\varepsilon}: \mathbb{R} \rightarrow \mathbb{R}^{2}$ be an isometric immersion with its image contained in the disk $D^{2}(\varepsilon)$. (This $s_{\varepsilon}$ can be seen as a long planar curve located $\varepsilon$-close to the origin. The existence of such an $s_{\varepsilon}$, however simple, is not at all trivial, and it is pivotal for the construction of isometric immersions.)

Let $\Phi_{\varepsilon}: X \times \mathbb{R} \rightarrow Y$ be the composition of the immersion $X \times \mathbb{R} \rightarrow X \times D^{2}(\varepsilon)$ defined by $(x, t) \mapsto(x, s(t))$ with the above isometric immersion $f_{\times 2}: X \times D^{2}(\varepsilon) \rightarrow$ $Y$.

Then take the composition of $\Phi_{\varepsilon}$ with the graph $\Gamma_{\phi}: X \rightarrow \mathbb{R}$ for $f_{\varepsilon}$.

Since the metric on $X$ induced by the map $\Gamma_{\phi}$ from $g \oplus d t^{2}$ on the cylinder $X \times \mathbb{R}$ equals $g+d \phi^{2}$ and since $\Phi_{\varepsilon}$ is an isometric immersion, the proof follows.

True isometric extensions from $X$ to $X \times D^{2}(\varepsilon)$ with the product metric $g \oplus\left(d r_{1}^{2}+d r_{2}^{2}\right)$ are hard to come by (we construct some in section 3.12), but smooth extensions, call them $f_{\times^{\prime} 2}: X \times \mathbb{R}^{2} \rightarrow Y$, which are isometric on the tangent bundle $T\left(X \times \mathbb{R}^{2}\right) \mid X$, i.e., such that

the induced metrics $\left(D f_{X^{\prime} 2}\right)^{\star}(h)$ equal $g \oplus\left(d r_{1}^{2}+d r_{2}^{2}\right)$ on the restriction of the tangent bundle of $X \times \mathbb{R}^{2} \supset X$, to $X$

may be plentiful whenever $q \geq n+2$.

In fact such an extension $f_{\times^{\prime} 2}: X \times \mathbb{R}^{2} \rightarrow Y$ exists if and only if the manifold $X$ admits two normal linearly independent vector fields in $Y \underset{f}{\subset} X$. Therefore,

if $U \subset X$ is a contractible, e.g., homeomorphic to the $n$-ball, then isometric immersions $X \rightarrow Y$ (restricted to $U$ ) extend to immersions

$$
\left(f_{U}\right)_{\times^{\prime} 2}: U \times \mathbb{R}^{2} \rightarrow Y
$$

that are isometric on $T\left(X \times \mathbb{R}^{2}\right) \mid U$.

Now let $\phi$ be a smooth $\left(C^{1}\right.$ will do) function on $X$ with support contained in $U$ and observe that

the above construction of adding $d \phi^{2}$ to the metric $g$ on $X$ in the proof of $\left[+d \phi^{2}\right]$ with the use of the isometric immersion $s_{\varepsilon}: \mathbb{R} \rightarrow \mathbb{R}^{2}$ makes sense with $\left(f_{U}\right)_{\times^{\prime} 2}$ instead of $f_{\times 2}$. 
We cannot claim any longer that the resulting map $f_{\varepsilon}: X \rightarrow Y$ exactly induces the metric $g+d \phi^{2}$ (unless $\left(f_{U}\right)_{\times^{\prime} 2}$ is isometric on $U \times D^{2}(\varepsilon)$ ) but only that

the induced metrics $\left(D f_{\varepsilon}\right)^{\star}(h)$ uniformly converge to $g+d \phi^{2}$ on $X$ for $\varepsilon \rightarrow 0$.

This, combined with the Kuratowski-Weyl-Nash decomposition, yields the following

$\left[g_{0}+g^{\prime}\right]_{\delta}$-Corollary. Let $f_{0}:\left(X, g_{0}\right) \rightarrow(Y, h)$ be an isometric $C^{1}$-immersion, let $\varepsilon(x), \delta(x)>0$ be arbitrarily small positive functions on $X$ (constant if $X$ is compact), and let $g^{\prime}$ be a positive definite quadratic form on $X$.

Then there exits a $C^{1}$-immersion $f_{\delta}: X \rightarrow Y$ such that

$\left(1^{\prime}\right) \operatorname{dist}_{Y}\left(f_{0}(x), f_{\delta}(x)\right) \leq \varepsilon(x)$ for all $x \in X$;

$\left(2^{\prime}\right)\left\|\left(D f_{\delta}\right)^{\star}(h)-\left(g_{0}+g^{\prime}\right)\right\|(x) \leq \delta^{\prime}(x)$ for all $x \in X$,

where $\|. .$.$\| stands for some norm on the space of quadratic forms, e.g., the one$ defined with $g$ itself. (Since the function $\delta^{\prime}(x)$ may be chosen arbitrarily small, a specific choice of such a norm plays no role.)

Nash $\left[g_{0}+g^{\prime}\right]$-Theorem. Let $f_{0}:\left(X, g_{0}\right) \rightarrow(Y, h)$ be an isometric $C^{1}$-immersion, and let $g^{\prime}$ be a continuous positive definite quadratic form on $X$.

If $\operatorname{dim}(Y) \geq \operatorname{dim}(X)+2$, then there exits a $C^{1}$-immersion, say $f^{\prime}: X \rightarrow(Y, h)$, such that the induced metric satisfies

$$
\left(D f^{\prime}\right)^{\star}(h)=g_{0}+g^{\prime}
$$

Proof. One may (obviously) assume that the $\left(\delta\right.$-small) difference $g_{1}^{\prime}=g_{0}+g^{\prime}-$ $\left(D f_{\delta}\right)^{\star}(h)$ in the above $\left[g_{0}+g^{\prime}\right]_{\delta}$-corollary is positive definite. Then this corollary applies to $f_{1}=f_{\delta}$ and $g_{1}^{\prime}$ instead of $f$ and $g^{\prime}$, next it applies to $f_{2}=\left(f_{1}\right)_{\delta_{1}}$ and $g_{2}^{\prime}=g_{1}^{\prime \prime}$, etc., such that the sequence of the induced metrics $\left(D f_{i}\right)^{\star}(h)$ converges to $g_{0}+g^{\prime}$ in the fine $C^{0}$-topology for $i \rightarrow \infty$.

(This is the strongest possible $C^{0}$-topology on the space of continuous metrics on $X$ that coincides with the uniform topology for compact $X$.)

But, a priori, one can only claim that the maps $f_{i}: X \rightarrow Y$ converge to a $\left(g_{0}+g^{\prime}\right)$-isometric Lipschitz map rather than a $C^{1}$-smooth one.

However, a little thought shows that the maps $f_{i}$ do $C^{1}$-converge, since the essential perturbations of our maps at all steps were obtained via graphs of certain $\varepsilon$-small maps $X \rightarrow \mathbb{R}^{2}$, where one has the following bound on the $C^{1}$-sizes of these perturbations.

Graphical $C^{1}$-Estimate. Let $\Gamma: X \rightarrow X \times \mathbb{R}^{k}$ be a $C^{1}$-map that equals the graph of a map $\phi$ from $X$ to an $\varepsilon$-ball $D^{k}(\varepsilon) \subset \mathbb{R}^{k}$.

Then the $C^{1}$-distance between $\Gamma$ and (the graph of) the zero map $x \mapsto(x, 0) \epsilon$ $X \times \mathbb{R}^{k}$ which equals the $C^{1}$-norm of $\phi$ is, obviously, bounded by the norm of the quadratic form induced on $X$ by $\phi$ from the Euclidean metric $d r_{1}^{2}+\cdots+d r_{k}^{2}$ on $\mathbb{R}^{k}$.

The proof of Nash's $C^{1}$-theorem is, thus, concluded.

Apology. One could condense the above proof of Nash's theorem to twentytwenty-five lines - perhaps fewer - with the "conformal twist" that was originally used by Nash instead of Kuratowski and Weyl.

On the other hand, writing the above proof in formulas would make it both long and not truly rigorous, with the reason for this being twofold.

Formulas fare well only if they perfectly and unambiguously match the idea that they intend to express. But since there is neither a preferred isometric immersion 
$\mathbb{R} \rightarrow D^{2}(\varepsilon)$ nor a preferred extension of a smooth map from a submanifold $X$ to a larger manifold (e.g., from $X$ to $X \times \mathbb{R}^{2}$ ) with prescribed differential on $X$, writing specific formulas for these kinds of constructions only serves to pollute mathematical texts with irrelevant information.

Another reason is that formulas are not suitable for expressing category theoretical concepts. For instance, a computational verification that the composition of several isometric immersions encoded by formulas is isometric may run to a half page computation that, being utterly trivial, would be unreadable 17

Question. Why, despite all this, do some authors regress to 18th century mathematical language in their exposition of 20th (and 21th) century ideas?

3.3. Kuiper's $C^{1}$-Stretch and Isometric $C^{1}$-Immersions $X^{n} \rightarrow Y^{n+1}$. Let us prove, following Kuiper (1955) [53, the above Nash $\left[g+g^{\prime}\right]$-theorem with the assumption $\operatorname{dim}(Y) \geq \operatorname{dim}(X)+2$ relaxed to $\operatorname{dim}(Y) \geq \operatorname{dim}(X)+1$.

Now instead of maps $\left(f_{U}\right)_{\times^{\prime} 2}: U \times \mathbb{R}^{2} \rightarrow Y$, we deal with

$$
\left(f_{U}\right)_{\times^{\prime} 1}: U \times \mathbb{R} \rightarrow Y
$$

for small open (ball-like) sets $U \subset X$ as earlier and with the $\operatorname{disc} D^{2}(\varepsilon)$ replaced by the segment $D^{1}(\varepsilon)=[-\varepsilon, \varepsilon] \subset \mathbb{R}$.

The point where Nash's proof stalls is non-existence of smooth isometric immersions $s_{\varepsilon}: \mathbb{R} \rightarrow D^{1}(\varepsilon)=[-\varepsilon, \varepsilon] \subset \mathbb{R}$ and, generically, say for $n \geq 3$, the metric $g+d \phi^{2}$ cannot be induced from the metric $g \oplus d r^{2}$ by a $C^{2}$-smooth 18 map $X \rightarrow X \times D^{1}(\varepsilon)$ for small $\varepsilon>0$.

However, one has, for all $\varepsilon>0$, and all $C^{1}$-functions $\phi$ on $X$ with compact supports, the following

Kuiper's Stretching Lemma. There exist $C^{1}$-smooth maps (that are embeddings) $\Gamma_{\varepsilon, \epsilon}: X \rightarrow X \times D^{1}(\varepsilon), \epsilon>0$, such that

$$
\left\|\left(D \Gamma_{\varepsilon, \epsilon}\right)^{\star}\left(g \oplus d r^{2}\right)-\left(g+d \phi^{2}\right)\right\| \rightarrow 0 \text { for } \epsilon \rightarrow 0,
$$

where $\|.$.$\| denotes some norm in the space of continuous quadratic forms on X$.

Granted this, the proof of the $\left[g+g^{\prime}\right]$-theorem proceeds word for word as it was with the $\left[+d \phi^{2}\right]$-lemma in the previous section. But, unlike anything we have met so far, the proof of this lemma needs a bit of geometric reasoning, which is presented below.

Smoothing the Corners. Let $Z=Z^{n+1}$ be a Riemannian manifold with its metric denoted by $\sigma$, and let $X=X^{n} \subset Z$ be a hypersurface that is smooth except for a corner $C=C^{n-1} \subset X$ where two smooth parts of $X$ meet transversally. (Technically speaking, transversality is unnecessary, but it helps to make a clear picture in mind.)

Observe that, despite the corner, the Riemannian metric induced by $\sigma$ on $X$ is continuous.

Given $\epsilon>0$ and $\delta>0$, let us smooth this corner by replacing the original piecewise smooth embedding, say $\Gamma: X \rightarrow Y$ by a $C^{1}$-embedding $\Gamma_{\epsilon}: X \rightarrow Y$, by slightly

\footnotetext{
${ }^{17}$ It is worse for functoriality: this single word may expand to several pages of $99 \%$ meaningless formulas.

${ }^{18}$ This, of course, makes sense only if $g$ and $\phi$ are $C^{2}$-smooth. On the other hand, there are (non-generic) obstructions in the $C^{1}$-category, e.g., where the levels of $\phi$ are minimal hypersurfaces in $(X, g)$.
} 
perturbing $\Gamma$ in the $\varepsilon$-neighbourhood of $C$, such that

(1) $\Gamma_{\epsilon}(x)=\Gamma(x)$ for $\operatorname{dist}_{Y}(x, C)>\epsilon$;

(2) the metric induced by $\Gamma_{\epsilon}$ remains close to the original one:

$$
\left\|\left(D \Gamma_{\epsilon}\right)^{\star}(\sigma)-(D \Gamma)^{\star}(\sigma)\right\| \leq \delta .
$$

We start by observing that the existence of such $\Gamma_{\epsilon}$ is obvious for curves in surfaces, i.e., for $n=1$, where the smoothing can be done smoothly depending on variable curves 19 Then the general case follows by applying this curve smoothing to the intersections of $X$ with small smooth $\operatorname{discs} D_{c}^{2} \subset Z$ normal to $C$ at all points $c \in C$.

The proof of Kuiper's lemma is instantaneous now with a piecewise linear map $s_{\varepsilon}: \mathbb{R} \rightarrow D^{1}(\varepsilon)=[-\varepsilon, \varepsilon] \subset \mathbb{R}$ that is isometric everywhere except for finitely many break points where the derivative of $s_{\varepsilon}$ switches from +1 to -1 .

The graph $\Gamma_{\varepsilon}: X \rightarrow(X, g) \times D^{1}(\varepsilon) \subset X \times \mathbb{R}$ of $\phi_{\varepsilon}$ is piecewise smooth with "corners" that correspond to the break points of $s_{\varepsilon}$, where these corners are smoothed as above with $\left(X \times \mathbb{R}, g \oplus d r^{2}\right)$ in the role of $(Z, \sigma)$.

The proof of Kuiper's lemma, and his isometric $C^{1}$-immersion theorem for $X^{n} \rightarrow$ $Y^{q}$ and for all $q \geq n+1$, is concluded.

(We did not pay much attention to the $C^{1}$-convergence of the sequence of maps $X \rightarrow Y$ obtained with Kuiper's lemma but this is (almost) obvious as in Nash's case. It is also obvious that if the original map $X \rightarrow Y$ was an embedding, then the new one with $g^{\prime}$ added to the induced metric remains an embedding.)

The above may seem shamefully easy; you may smile at the geometers who, for years, tried to prove that isometric $C^{1}$-immersions, say of surfaces with $C^{\infty}$-metrics $g$ with positive curvatures to the 3 -space, must be $C^{\infty}$-smooth and, hence, convex. Well, mathematics teaches us humility; another Nash may come up with something equally "obvious" that you have believed all your life to be impossible.

3.4. The Proof of the Smale-Hirsch $h$-Principle by Nash-Kuiper Stretching. Unbelievable isometric $C^{1}$-immersion theorems were followed by Smale's equally amazing turning sphere inside out (1958) 68 followed by the immersion theory for $S^{n} \rightarrow \mathbb{R}^{q}$ (Smale 1959, 69]), and for $X^{n} \rightarrow Y^{q}, q \geq n+1$, in general (Hirsch 1959, 45]) 20

It is also amazing how the homotopy theoretic way of thinking, introduced to the immersion theory by Smale and combined with a most primitive version of NashKuiper stretching, delivers a short proof of all results of the smooth immersion theory. In fact all of this theory can be painlessly derived from the following procedure.

Turning $X \subset Y$ Normally to a Subbundle $\theta \subset T(Y)$. Let $Y$ be a split Riemannian manifold, let $Y=\left(Y_{1} \times Y_{2}, h_{1} \oplus h_{2}\right)$, and let a submanifold $X \subset Y$ be "transversal", meaning here nowhere tangent, to the subbundle $\theta_{2} \subset T(Y)$ of the tangent spaces to the $Y_{2}$ - "fibres" $y_{1} \times Y_{2} \subset Y$.

Our transversality is equivalent to the projection $f_{1}$ of $X$ to $Y_{1}$ being an immersion, and if $\operatorname{dim}\left(Y_{1}\right) \geq \operatorname{dim}(X)$, one can "strengthen" this transversality by deforming $f_{1}$ to another immersion, say $f_{1}^{\prime}: X \rightarrow Y_{1}=\left(Y_{1}, h_{1}\right)$ that induces on $Y_{1}$ a metric $\left(D f_{1}^{\prime}\right)^{*}\left(h_{1}\right)=\left(D f_{1}\right)^{*}\left(h_{1}\right)+g^{\prime}$ where $g^{\prime}$ is VERY LARGE.

\footnotetext{
${ }^{19}$ Some corners can and some cannot be smoothed without moving such a corner 48 .

${ }^{20}$ The history of this discovery is creatively laid out by David Spring in [71], where one finds an extensive bibliography.
} 
Then, clearly the map $f=\left(f_{1}^{\prime}, f_{2}\right): X \rightarrow Y$, for the projection $f_{2}: X \rightarrow Y_{2}$ which has not been modified, becomes almost normal to the subbundle $\theta_{2}$.

(Neither Weyl-Kuratowski-Nash nor the full strength of the Nash-Kuiper1 stretching construction is needed here, but only a possibility of unrestricted stretching $X \subset Y_{1}$ in all directions.)

Let, more generally, $Y=(Y, h)$ be a Riemannian manifold, with a given normal splitting of its tangent bundle, $T(X)=\theta_{1} \oplus \theta_{2}$, and let $X \subset Y$ be a smooth submanifold transversal to $\theta_{2}$.

If $\operatorname{rank}\left(\theta_{1}\right)>\operatorname{dim}(Y)$, then there exists a smooth isotopy of embeddings $f_{t}: X \rightarrow$ $Y, 0 \leq t \leq 1$, such that $f_{0}=f$ and -

1. $\operatorname{dist}_{Y}\left(f_{t}(x), x\right)$ is bounded by a given positive continuous function $\varepsilon(x)$ on $X$ for all $0 \leq t \leq 1$;

2. the embeddings $f_{t}: X \rightarrow Y$ are transversal to $\theta_{2}$ for all $0 \leq t \leq 1$;

3. the embedding $f_{t=1}: X \rightarrow Y$ is $\delta$-normal to $\theta_{2}$ for a given positive continuous function $\delta(x)$ on $X$, which means that the scalar products between the unit tangent vectors to $f_{t=1}(X) \subset Y$, that is to $X$ embedded to $Y$ by $f_{t=1}$, at the points $f_{1}(x) \in Y$ and such vectors in the (vector) fibres $\left(\theta_{2}\right)_{f_{1}(x)}$ of $\theta_{2}$ are bounded by $\delta(x)$.

Proof. Given a point $y \in Y$ and $\epsilon>0$, there exists a neighbourhood $U_{\epsilon}(y) \subset Y$ of $y$ such that the Riemannian metric on $Y$ and the splitting of the tangent bundle can be $\epsilon$-approximated by an actual splitting $\left(U_{\epsilon, 1} \times U_{\epsilon, 2}, h_{1} \oplus h_{2}\right)$ of $U_{\epsilon}(y)$.

Since the Nash-Kuiper stretching can be (obviously by its construction) localised near a given point $x \in X \subset Y$, the above $Y=Y_{1} \times Y_{2}$ case implies the general one. (Alternatively, one could apply the Nash-Kuiper stretching directly in the directions normal to $\theta_{2}$.)

Homotopy Lifting of Transversality. Let $\theta_{t}, t \geq 0$, be a continuous family of subundles in the tangent bundle $T(Y)$ of a smooth manifold $Y$, and let $f_{0}: X \rightarrow Y$ be a smooth immersion (embedding) transversal to $\theta_{0}$, where $X$ is another smooth manifold.

If $q=\operatorname{corank}\left(\theta_{0}\right)=\operatorname{dim}(X)-\operatorname{rank}\left(\theta_{0}\right) \geq \operatorname{dim}(X)+1$, then there exists a $C^{1}$ continuous family of smooth immersions (embeddings) $f_{t}: X \rightarrow Y$ that are transversal to $\theta_{t}$ for all $t \geq 0$, where, moreover, one can have all $f_{t}$ arbitrarily close to $f_{0}$ in the uniform (fine $C^{0}$ for non-compact $X$ ) topology,

$$
\operatorname{dist}_{Y}\left(f_{t}(x), x\right) \leq \varepsilon(x) .
$$

Proof. Fix some Riemannian metric on $Y$, and keep turning $X \rightarrow Y$ at the moments $t$ almost normally to $\theta_{t}$, where this $\theta_{t}$ with its orthogonal complement orthogonally splits $T(Y)$ for all $t$.

Now we are able to prove the following

(Corollary to) Hirsch's Approximation Theorem. Let the dimensions of smooth manifolds $X$ and $Y$ satisfy $\operatorname{dim}(Y) \geq \operatorname{dim}(X)+1$. Then

a continuous map $f_{0}: X \rightarrow Y$ admits a uniform (fine $C^{0}$ for non-compact $X$ ) approximation by smooth immersions $X \rightarrow Y$ if and only if $f_{0}$ is homotopic to an immersion $X \rightarrow Y$. 
For instance, such an approximation is possible for all $\varphi_{0}$ if $X$ is diffeomorphic to the Euclidean space $\mathbb{R}^{n}$ for $n<q=\operatorname{dim}(Y) 21$

Proof. Let us approximate the continuous homotopy of $f_{0}$ to an immersion by a smooth one and observe that the graph of this is a smooth isotopy $\Gamma_{t}: X \rightarrow X \times Y$ of the graph $\Gamma_{0}: X \rightarrow X \times Y$ of $f_{0}$ to an embedding $\Gamma_{1}$ that is transversal to the $X$-fibres of the projection $p: X \times Y \rightarrow Y$.

Extend this isotopy to an isotopy, say $\tilde{\Gamma}_{t}: U \rightarrow X \times Y$, for a small neighbourhood $U \subset X \times Y$ of $\Gamma_{0}(X) \subset X \times Y$ and pull back the $X$-fibres by $\Gamma_{t}$ to $U \supset X$.

Then by "homotopy lifting of transversality" applied to the subbundles $\theta_{t} \subset T(U)$ that are tangent to these pull-backs, there exists an isotopy $\Gamma_{t}^{\prime}: X \rightarrow U, 0 \leq t \leq 1$, for $\Gamma_{0}^{\prime}=\Gamma_{0}: X \rightarrow U$ that keeps as $C^{0}$-close to $\Gamma_{0}$ as one wishes and that brings $X$ for $t=1$ to a position where it is transversal to the $X$-fibres of the projection $X \times Y \rightarrow Y$.

Then the projection of $\Gamma_{1}^{\prime}$ to $Y$, that is the map $p \circ \Gamma_{1}^{\prime}: X \rightarrow Y$, serves as the required immersion approximating $f_{0}: X \rightarrow Y$.

Similarities and Dissimilarities Between the Two Proofs. The above argument is not as much removed from the original idea by Smale as it may seem:

1. The "proof by stretching" fundamentally depends on lifting of homotopies, that is the Serre fibration property for maps between certain function spaces, as much as the Smale-Hirsch arguments rely on flexibility of the sheaf of immersions $\{U \rightarrow Y\}_{U \subset X}$ from open subsets $U \subset X$ to $Y$ that (essentially) is the Serre fibration property for the restriction maps between spaces of immersions,

$$
\operatorname{Imm}\left(X_{1} \rightarrow Y\right) \rightarrow \operatorname{Imm}\left(X_{2} \rightarrow Y\right), X_{2} \subset X_{1} \subset X, \text { for } \operatorname{dim}(Y)>\operatorname{dim}(X) .
$$

This flexibility, proven by Smale, implies, by a homotopy theoretic argument of Smale and Hirsch, the following property of

the differential $D: f \mapsto D f$ of smooth maps $f: X \rightarrow Y$ that, by the definition of "immersion", sends the space of immersions $\operatorname{Imm}=\operatorname{Imm}(X \rightarrow Y)$ to the space $\operatorname{Inj}=\operatorname{Inj}(T(X) \rightarrow T(Y))$ of continuous fibrewise linear and fibrewise injective maps between the tangent bundles.

\section{The $h$-PRINCIPLE FOR IMMERSIONS}

If $\operatorname{dim}(Y) \geq \operatorname{dim}(X)+1$, then the map

$$
D: \operatorname{Imm} \rightarrow \operatorname{Inj}
$$

is a homotopy equivalence.

In fact Smale and, following him, Hirsch prove the weak homotopy equivalence property of $D$, which means that the induced homomorphisms between the homotopy groups, denoted $D_{i}: \pi_{i}(\mathrm{Imm}) \rightarrow \pi_{i}(\mathrm{Inj})$, are isomorphisms for all $i=0,1,2, \ldots$, where this "weak" is as good as "strong" for the present discussion.

This is derived from flexibility by induction on dimension, where the isomorphism property of $D_{i}, i=0,1, \ldots k$, for immersions of $m$-dimensional (sub)manifolds $X_{1} \subset$ $X$ follows from this property for immersions of $(m-1)$-dimensional (sub)manifolds $X_{2} \subset X_{1}$ and $i=0,1, \ldots, k+1$ by the exactness of the homotopy sequence for the restriction maps

$$
\operatorname{Imm}\left(X_{1} \rightarrow Y\right) \rightarrow \operatorname{Imm}\left(X_{2} \rightarrow Y\right)
$$

\footnotetext{
${ }^{21}$ The latter statement is beautiful in its simplicity. I challenge anybody to find an independent proof of this, where a non-trivial point that makes a truly direct construction impossible is the condition diffeomorphic to $\mathbb{R}^{n}$.
} 
Thus - this is the essence of Smale's approach to immersions - his argument when applied, for instance, to the proof of the existence of immersions relies on a use of higher homotopy groups of spaces of immersions of lower dimensional manifolds. But the proof by the Nash-Kuiper stretching directly applies to all homotopies simultaneously with no $k \mapsto k+1$ shift.

2. The proofs of the flexibility of immersions by Smale as well as of the homotopy lifting by Nash-Kuiper stretching are established not with the use of some specific geometric features of immersions but, on the contrary, by wiping out all traces of such properties by "massively bending and wrinkling" these immersions, where the wrinkling performed with stretching is more extensive than the more economical wrinkling used by Smale to the point that stretched submanifolds $X \subset Y$ start looking kind of "fractal" on a certain scale 22

3. The original $C^{1}$-arguments by Nash and Kuiper as well as those by Smale and Hirsch have been overshadowed by simpler and more general proofs of the results these methods were originally designed for 23 But these arguments may fare better than more advanced ones in certain cases.

For instance,

- a Smale-Hirsch style argument, in conjunction with Nash's perturbation/implicit function theorem, yields, under certain restrictions on dimensions, flexibility, hence, the $h$-principle, for the sheafs of $C^{\infty}$-smooth free isometric immersions between pseudo-Riemannian manifolds (see 3.3.2 in 35]));

- Nash's twist applies to symplectic imbedding (3.4.2 in [35]), to symplectic isometric $C^{1}$-embeddings and immersions [19] to contact and to Cartnot-Carathéodory isometric $C^{1}$-immersions [17, where the convex integration in its present form is inapplicable.

3.5. Convex Integration. The logic of the Nash-Kuiper construction of isometric $C^{1}$-immersions by successive stretchings and the issuing $h$-principle become fully transparent in the general setting of the so-called convex integration as follows.

Regard $C^{1}$-maps $X=\underline{X} \times \mathbb{R} \rightarrow Y$ as curves that are maps from $\mathbb{R}$ to the space of $C^{1}$-maps $\underline{X} \rightarrow Y$, and visualise the Nash-Kuiper stretchings in the $\mathbb{R}$-direction as certain modifications of these curves.

What is most essential is that this modification by stretching may be performed, speaking in terms of curves, by arbitrarily small perturbations of these curves with respect to the $C^{1}$-distance in the space of $C^{1}$-maps $\underline{X} \rightarrow Y$.

$C^{\perp}$-Topology. This, now in terms of maps $X \rightarrow Y$, signifies that the correspondingly modified (by stretching) maps $X=\underline{X} \times \mathbb{R} \rightarrow Y$ (may be chosen to) stay arbitrarily $C^{1}$-close to the original unstretched ones on the hypersurfaces $\underline{X} \times t \subset X=\underline{X} \times \mathbb{R}$.

This is called approximation in $C^{\perp}$-topology (here $C^{\perp}=C^{\perp_{1}^{0}}$ ) that is $C^{0}$ in the $\mathbb{R}$-directions, i.e., on the lines $x \times \mathbb{R} \subset X \times \mathbb{R}, x \in X$, and it is $C^{1}$ on $\underline{X} \times t$.

\footnotetext{
${ }^{22}$ This fractality of isometric $C^{1}$-immersions is made visible in [10]. Possibly, one can rigidify the $C^{1}$-theory by replacing, say, $C^{1, \alpha}$-regularity conditions by some kind of self-similarity of the differentials of isometric maps.

${ }^{23}$ The conceptual approach to isometric $C^{1}$-immersion proceeds via convex integration, while the shortest known proof of the Hirsch immersion theorem for $X^{n} \rightarrow \mathbb{R}^{q}, q>n$ is delivered by removal of singularities introduced in 1971 by Eliashberg and the author. This method uses a minimal amount of "wrinkling" and allows approximation of smooth maps by immersions in certain Sobolev $W^{r, p}$-topologies. See 2.1.1 in 35 and references therein.
} 
Splitting and Localisation. Stretchings of maps $f: X \rightarrow Y$, as it is done by Nash and Kuiper, are supported in small neighbourhoods $U \subset X$. These $U$ may be assumed diffeomorphic to balls, and the restricted maps $f_{\mid U}: U \rightarrow Y$ can be interpreted as curves with splittings $U=\underline{U} \times \mathbb{R}$ adapted to required modifications of maps $f: X \rightarrow Y$ on $U$.

In the following sections 3.5.1-3.5.4 we describe the essential constituents of such modifications in a simplified form.

3.5.1. Oscillatory Curve Approximation. Let $\Upsilon$ be a Banach space, e.g., $\Upsilon=\mathbb{R}^{q}$, let $E \subset \Upsilon$ be a path connected subset, and let intconv $(E) \subset \Upsilon$ denote the interior of the convex hull of $E$.

[M] If the derivative $\frac{d f_{0}(t)}{d t} \in \Upsilon$ of a $C^{1}-\operatorname{map} f_{0}: \mathbb{R} \rightarrow \Upsilon$ is contained in $\operatorname{intconv}(\Upsilon)$ for all $t \in \mathbb{R}$, then $f_{0}$ can be finely $C^{0}$-approximated by $C^{1}$-maps $f: \mathbb{R} \rightarrow \Upsilon$ with their derivatives contained in $E$,

$$
\frac{d f(t)}{d t} \in E \text { for all } t \in \mathbb{R} \text {. }
$$

Almost Proof. Such approximation by piecewise smooth, in fact, by piecewise linear, maps $\check{f}: \mathbb{R} \rightarrow \Upsilon$ is immediate without the connectivity condition for $\Upsilon$.

And if the two values, $\check{e}_{\text {left }}, \check{e}_{\text {right }} \in E$ of the one-sided derivatives $\frac{d \check{f}(t)}{d t}$ of such an $\check{f}$ at some $t_{0} \in \mathbb{R}$ are joined by a continuous path $P_{0} \subset \Upsilon$, then one can easily approximate $\check{f}$ by a smooth $f$ that equals $\check{f}$ outside a small interval $\left[t_{0}-\varepsilon_{0}, t_{0}+\varepsilon_{0}\right] \subset$ $\mathbb{R}$ and such that

$$
\frac{d f(t)}{d t} \in P_{0} \text { for } t_{0}-\varepsilon_{0} \leq t \leq t_{0}+\varepsilon_{0},
$$

where the path $P_{0}$ joining the two points must be taken in $E$ which is possible under our connectivity assumption.

Why Oscillatory? If, for instance, $f_{0}: \mathbb{R} \rightarrow \Upsilon$ is a linear map with a constant derivative, say $\frac{d f_{0}(t)}{d t} \epsilon \operatorname{conv}(E) \subset \Upsilon$ that is not contained in $E$, then in order to keep close to $f_{0}$, maps $f: \mathbb{R} \rightarrow \Upsilon$ with derivatives in $E$, must go back and forth frequently changing direction of motion without ever coming close to $\frac{d f_{0}(t)}{d t}$.

Looking Two Steps Ahead. The above looks childishly simple, yet in conjunction with another (almost as) simple fact-convex decomposition - it leads to rather paradoxical solvability of generic underdetermined systems of equations and of a class of determined and overdetermined systems including those satisfied by isometric immersions $X^{n} \rightarrow Y^{q}, q>n$, which are overdetermined for $q<s_{n}=\frac{n(n+1)}{2}$.

Broken and Unbroken Convex Integration: Analysis Versus Topology. An analyst would regard "connectivity" in the formulation of the lemma as a mere nuisance and would attribute the term "convex integration" to whatever follows from the piecewise smooth version of the approximation lemma, e.g., the existence of Lipschitz isometric maps of all Riemannian $n$-manifolds to $\mathbb{R}^{n}$.

(See 2.4.11 in [35] and 59] for applicantions of this "broken convex integration" to the classical PDE.)

But from a topologist's point of view, this "disconnected stuff" is of little interest since all $h$-principles collapse when the continuity of the derivatives is broken.

Of course, the mere existence theorems remain valid; every continuous map $X \rightarrow$ $Y$ can be approximated by piecewise smooth immersions for $\operatorname{dim}(Y) \geq \operatorname{dim} X$, but 
no self-respecting topologist would give the status of "theorem" to anything that is, homotopy theoretically speaking, 99\% vacuous and-this is a minor pointtechnically trivial.

To avoid confusion, we reserve the name "convex integration" for its continuous version that came up in the ambience of homotopy theory, and we call its discontinuous rendition(s) broken convex integration.

Differential Inclusions. The curve approximation regarded as "convexification" of discontinuous/multivalued ordinary differential equations, apparently, goes back to A. F. Filippov, but the path differential inclusions followed afterwards has diverged in a direction far removed from convex integration 24 In fact, the latter is predominantly concerned with partial rather than ordinary differential relations and the overlap between the two theories is limited to a single, essentially obvious, lemma. (But this may (?) change in the future.)

3.5.2. Convex Decomposition. Let $E \subset \mathbb{R}^{q}$ be a connected $C^{r}$-submanifold, $r \geq 1$, and let $f_{0}: X \rightarrow \operatorname{intconv}(E) \subset \mathbb{R}^{q}$ be a $C^{r}$-map of a compact manifold $X$ to the interior of the convex hull of $E$. Then

$\left[\bigcirc \sim \odot\right.$ the map $f_{0}$ equal to the convex combination of $C^{r}$-maps $X \rightarrow E$,

$$
f_{0}=\sum_{l} p_{i} \varphi_{l}, l=1,2,3, \ldots, m,
$$

where $\varphi_{l}: X \rightarrow E$ are $C^{r}$, and $p_{l}$ are positive numbers, such that $\sum_{l} p_{l}=1$.

(Nothing of the kind is true for disconnected subsets E, e.g., for finite ones.)

Proof. It is obvious (by partition of unity, where no connectedness of $E$ is required) that there exist $C^{r}$-maps $\phi_{i}$ and positive $C^{r}$-functions $\pi_{i}(x)$ (rather than constants $p_{i}$ ) such that $f_{0}=\sum_{i} \pi_{i} \phi_{i}$.

Since $E$ is path-connected, there exists a $C^{r}$-smooth map $\Phi: X \times[0,1] \rightarrow E$, such that $\Phi\left(x, t_{i}\right)=\phi_{i}(x)$ for some $t_{i} \in[0,1]$.

Regard the sets of points $\left\{t_{i}\right\} \subset[0,1]$ with the weights $\pi_{i}(x)$ assigned to $t_{i}$ as probability measures on the segments $x \times[0,1] \subset X \times[0,1]$, denoted $d \pi_{x} t$, where, clearly,

$$
\int_{0}^{1} \Phi(x, t) d \pi_{x} t=\sum_{i} \pi_{i}(x) \phi_{i}(x)=f_{0}(x) .
$$

Then (this is obviously possible) approximate $d \pi_{v} t$ by $C^{r}$-smooth strictly positive measures, such that also $C^{r}$-smoothly depend on $x \in X$, say $d \pi_{x}^{\circ} t$, that satisfy the same relation

$$
\int_{0}^{1} \Phi(x, t) d \pi_{x}^{\circ} t=f_{0}(x) .
$$

Now - this is the crucial moment where we switch from functions $\pi_{i}(x)$ to constants $p_{l}$-let

$A: X \times[0,1] \rightarrow X \times[0,1]$ be a $C^{r}$-map that sends the intervals $x \times[0,1] \subset X \times[0,1]$ to themselves and that

pushes forward the Lebesgue measure $d t$ from these intervals to $d \pi_{x}^{\circ} t 25$

${ }^{24}$ See http://link.springer.com/chapter/10.1007/978-3-540-44398-8_3\#page-1. But I must admit I have not looked closely at this subject matter.

${ }^{25}$ These $A$ obviously exist for all connected manifolds in place of $[0,1]$ and, back to $[0,1]$, such an $A$ is uniquely, up to $t \mapsto 1-t$, determined by the measures $d \pi_{x}^{\circ} t$. 
Clearly, the composed map $\Psi(x, t)=(\Phi \circ A)(x, t): X \times[0,1] \rightarrow E$ satisfies

$$
\int_{0}^{1} \Psi(x, t) d t=f_{0}(x)
$$

and approximation of this integral by Riemann sums, shows that

the convex combinations of $C^{r}$-maps $X \rightarrow E$ are $C^{r}$-dense in the space of $C^{r}$-maps $X \rightarrow \operatorname{intconv}(E)$.

Finally, since the $C^{r}$-submanifold $E \subset \mathbb{R}^{q}, r \geq 1$, is connected, its tangent spaces (brought to the origin) linearly span $\mathbb{R}^{q}$; therefore, by the elementary implicit function theorem,

the interior of the convex hull of $C^{r}$-maps $X \rightarrow E$ is open in the space of $C^{r}$-maps $X \rightarrow \operatorname{intconv}(E)$.

Hence,

all $C^{r}$-maps from $X$ to the interior of the convex hull $\operatorname{conv}(E) \subset \mathbb{R}^{q}$ are

convex combinations of $C^{r}$-maps $X \rightarrow E$. QED

24 Questions. What are non- $C^{1}$-smooth (connected) subsets $E \subset \mathbb{R}^{q}$ for which the subset $\operatorname{intconv}\left(C^{0}(X \rightarrow E)\right) \subset C^{0}(X \rightarrow \operatorname{intconv}(E))$ is open?

Is there a reasonable bound on the number of summands in $f_{0}=\sum_{l} p_{l} \phi_{l}$ ?

\subsection{3. $C^{\perp}$-Approximation .}

Notation. Given smooth manifolds $X$ and $Y$, let $H=\operatorname{hom}(T(X) \rightarrow T(Y))$ be (the total space of) the vector bundle over $X \times Y$ with the fibres

$$
H_{x, y}=\operatorname{hom}\left(T_{x}(X) \rightarrow T_{y}(Y)\right) \subset H,(x, y) \in X \times Y .
$$

Suppose our manifold $X$ splits, $X=\underline{X} \times \mathbb{R}$, and let

- $\underline{T}=\underline{T}(X)$ be the (total space of) the bundle of subspaces $\underline{T}_{x} \subset T_{x}(X)$ that are tangent to the $\underline{X}$-fibres $\underline{X} \times t \subset X \times \mathbb{R}$;

- $T^{\mid}=T^{\mid}(X)$ be the (total space of) the bundle of subspaces $T_{x}^{\mid}=T_{x}^{\mid}(X) \subset T_{x}(X)$ that are tangent to the lines $\underline{x} \times \mathbb{R} \subset \underline{X} \times \mathbb{R}$;

- $\underline{H}_{x, y}=\operatorname{hom}\left(\underline{T}_{x} \rightarrow T_{y}(Y)\right)$;

- $H_{x, y}^{\mid}=\operatorname{hom}\left(\underline{T}_{x}^{\mid} \rightarrow T_{y}(Y)\right)$, where $H_{x, y}^{\mid}=T_{y}(Y)$, since $T_{x}^{\mid}=\mathbb{R}_{x}=\mathbb{R}$.

In these terms, $\Delta_{x, y}=\underline{H}_{x, y} \oplus H_{x, y}^{\mid}$and, accordingly, the differentials of maps $f: X \rightarrow Y$ decompose to pairs

$$
D f(x)=\left(\underline{D} f(x), \frac{\partial f(x)}{\partial t}\right),
$$

where $\underline{D} f \in \underline{H}_{x, f(x)}$ denotes the restriction of $D f$ to $\underline{T}$ and

$$
\frac{\partial f(x)}{\partial t} \in H_{x, f(x)}^{\mid}=T_{f(x)}(Y)
$$

is the derivative in the $t$-direction.

Given a subset $E_{x, y} \subset H_{x, y}$ and a linear map $\underline{h} \in \underline{H}_{x, y}$, define

$$
E_{\underline{h}}^{\mid} \subset H_{x, y}^{\mid}=T_{y}(Y)
$$

by the following condition:

if $f: X \rightarrow Y$ is a smooth map such that $\underline{D} f(x)=\underline{h}$, then

$$
\frac{\partial f(x)}{\partial t} \in E_{\underline{h}}^{\mid} \Leftrightarrow D f(x) \in E_{x, y} \text { for } y=f(x) .
$$


(In terms of linear algebra, $E_{\underline{h}}^{\mid} \subset H_{x, y}^{\mid}$is equal to the set of those homomorphisms $\tau \in H_{x, y}^{\mid}$for which the homomorphism $T_{x}(X) \rightarrow T_{y}(Y)$ defined by the pair $(\underline{h}, \tau)$ is contained in $E_{x, y}$.)

Now we are ready to proceed with $C^{\perp}$-APPROXIMATION.

Let

$$
E=\bigcup_{x, y} E_{x, y} \subset H=\bigcup_{x, y} H_{x, y}
$$

be a locally closed subset, let $f_{0}: X \rightarrow Y$ be a $C^{1}$-map, and suppose that the subsets $E_{D f(x)}^{\mid} \subset T_{y}(Y)$ "continuously depend" on $x \in X$.

This means that the natural projection

$$
E_{f_{0}}^{\mid}=\operatorname{def} \bigcup_{x \in X} E_{\underline{D} f_{0}(x)}^{\mid} \rightarrow X
$$

is a topological fibration 26 where this projection comes from that of the (total space of the) " $f_{0}$-lift" of the bundle $T(Y)$ to $X$, for

$$
E_{f_{0}}^{\mid} \subset f_{0}^{!}(T(Y)) \rightarrow X
$$

Let

(a) the subsets $E_{D f(x)}^{\mid} \subset T_{y}(Y)$ be path-connected for all $x \in X$ and

(b) the derivatives $\frac{\partial f_{0}(x)}{\partial t} \in T_{f_{0}(x)}(Y)$ be contained in the convex hulls

$$
\operatorname{conv}\left(E_{\underline{D} f(x)}^{\mid}\right) \subset T_{y}(Y),
$$

for all $x \in X$.

[ $\perp$ Then, for an arbitrary neighbourhood $\Omega=\Omega_{H} \subset H$ of the subset $E \subset H$, there exists a fine $C^{\perp}$-approximation of $f_{0}$ by $C^{1}$-maps $f: X \rightarrow Y$, the differentials of which, $D f(x) \in H_{x, y} \subset H$, are contained in $\Omega$ for all $x \in X$.

Almost Proof. Start by upgrading (b) to

$$
\frac{\partial f_{0}(x)}{\partial t} \in \operatorname{intconv}\left(E_{\underline{D} f(x)}^{\mid}\right)
$$

by an arbitrarily small perturbation of $E$.

Then a generalised version of convex decomposition (see [ $\sim \odot]$ in the previous section) goes along with a suitable generalisation of the oscillatory curve approximation (see [M] in 3.5.1) to $f_{0}: X=\underline{X} \times \mathbb{R} \rightarrow Y$ regarded as an " $\mathbb{R}$-curve" in the space of maps $\underline{X} \rightarrow Y$ for $t \mapsto f_{0} \mid \underline{X} \times t: \underline{X}=\underline{X} \times t \rightarrow Y$.

(Formulation and application of the needed generalisations of $[\bigcirc \sim 0$ ] and $[\mathrm{N}]$ are quite apparent as well as the necessary modifications of their proofs.)

\subsubsection{Convex Hull Rendition of Nash-Kuiper Stretching.}

Gradient Splitting. Let $Y=(Y, \tilde{g})$ be a Riemannian manifold 27 let $f_{0}: X \rightarrow Y$ be an immersion, and let $\phi: X \rightarrow \mathbb{R}$ be a $C^{1}$-function that has no critical points. Denote by $g_{0}$ the Riemannian metric on $X$ induced by $f_{0}$ from $\tilde{g}$ and assume that the gradient of $\phi$ splits $X$.

\footnotetext{
${ }^{26}$ In fact, one needs a weaker "continuity" than being a fibration; see 2.4 in 35 .

${ }^{27}$ We use the notation " $\tilde{g}$ " instead of " $h$ " in order not to confuse it with homomorphisms $h: T(X) \rightarrow T(Y)$.
} 
This means that $X$ splits as $X=\underline{X} \times \mathbb{R}$ where the projection $X \rightarrow \mathbb{R}$ equals $\phi$ and where this splitting is $g_{0}$-normal, that is the lines $\underline{x} \times \mathbb{R} \subset X$ are $g_{0}$-orthogonal to the hypersurfaces $\phi^{-1}(t)=\underline{X} \times t \subset X$; thus, they are the gradient lines of $\phi$.

Notice that if $\phi: X \rightarrow \mathbb{R}$ has no critical points and if the submanifolds $\phi^{-1}(t) \subset X$ are compact 28 then the gradient of $\phi$ splits $X$.

Let $g^{\prime}$ be a semipositive definite quadratic differential form on $X$ that vanishes on the hypersurfaces $\underline{X} \times t \subset X$, and let

$$
E_{x, y}^{\prime}=E_{x, y}^{\prime}\left(f_{0}, g^{\prime}, \tilde{g}\right) \subset H_{x, y}=\operatorname{hom}\left(T_{x}(X) \rightarrow T_{y}(Y)\right.
$$

be the set of isometric linear maps $T_{x}(X) \rightarrow T_{y}(Y)$ with respect to the metrics $g_{0}+g^{\prime}$ on $T(X)$ and $\tilde{g}$ on $T(Y)$.

Then the subset

$$
E_{\underline{h}}^{\prime \mid} \subset H_{x, y}^{\mid}=T_{y}(Y)
$$

(as defined in the previous section) is either empty or it is equal to the $(q-n)$ sphere of radius $\left.R^{\prime}=\|\tau\|_{g^{\prime}} / \| \tau\right) \|_{\tilde{g}}, \tau \in T^{\mid}(x)$, positioned in the tangent space $T_{y}(Y)$ normally to the image of $D f\left(\underline{T}_{x}\right): \underline{T}_{x} \rightarrow T_{y}(Y)$,

$$
E_{\underline{h}}^{\prime \mid}=S^{q-n}\left(R^{\prime}\right) \subset T_{y}(Y) \ominus D f\left(\underline{T}_{x}\right), n=\operatorname{dim}(X), q=\operatorname{dim}(Y),
$$

where $E_{\underline{h}}^{\prime \mid}$ is non-empty if and only if the linear map $\underline{h}: \underline{T}_{x} \rightarrow T_{y}(Y)$ is isometric. That is, the metric induced from $\tilde{g}$ on the subspace $\underline{T}_{x} \subset T_{x}(X)$-which is, we recall, tangent to $\underline{X} \times t$ at $x=(\underline{x}, t)$-is equal to $g_{0}$.

Since $g_{0}=\left(D f_{0}\right)^{\star}(\tilde{g})$, the sets $E_{\underline{h}}^{\prime \mid}$ are non-empty for $\underline{h}=\underline{D} f_{0}(x)$ for all $x \in X$ and, clearly, the $t$-derivative of $f_{0}$ is contained in the convex hull of $E_{\underline{D} f(x)}^{\prime \mid}$ for all $x \in X$,

$$
D f_{0}(x) \in \operatorname{conv}\left(E_{\underline{D} f(x)}^{\prime \mid}\right) .
$$

Therefore, if $\operatorname{dim}(Y) \geq \operatorname{dim}(X)+1$, then the spheres $S^{q-n}(R)=E_{x}^{\mid}$have positive dimension and, hence, are connected, and the $C^{\perp}$ approximation from the previous section (see $[\perp]$ in there) applies and shows that

[*] $f_{0}$ admits a fine $C^{\perp}$-approximation by $C^{1}$-immersions $f: X \rightarrow Y$ such that the metrics $(D f)^{\star}(\tilde{g})$ induced by these immersions $f$ finely $C^{0}$-approximate $g_{0}+g^{\prime}$ on $X$ with an arbitrarily given small error.

Next we observe - this is in the category of "obvious" - that all continuous positive definite quadratic differential forms (Riemannian metrics) $g^{\prime}$ on $X$ can be decomposed as follows 29

[ $\left.\square^{\prime}\right] \sum \psi_{\nu} d \phi_{\nu}^{2}$-Decomposition. There exists a locally finite covering of $X$, that depends on $g^{\prime}$, by (arbitrarily small) smoothly split neighbourhoods $U_{\nu}=\underline{U}_{\nu} \times \mathbb{R}$, where all $\underline{U}_{\nu}$ are diffeomorphic to the sphere $S^{n-1}$ and such that the metric $g^{\prime}$ decomposes as

$$
g^{\prime}=\sum_{\nu} \psi_{\nu}(x) d \phi_{\nu}(x)^{2}
$$

where $\phi: U_{\nu}=\underline{U}_{\nu} \times \mathbb{R} \rightarrow \mathbb{R}$ are projections to the second coordinates and $\psi_{\nu}(x)$ are positive continuous functions on $X$ with supports in $U_{\nu} 30$

28 "Compact" here means compact without a boundary.

${ }^{29}$ Open problems related to this are formulated in section 3.9.

${ }^{30}$ This goes back to Nash's 1954 paper. It was used, instead of the Kuratowski-Weyl decomposition, by Nash and Kuiper for construction of isometric $C^{1}$-embeddings. Also this decomposition, 
Since addition of $\psi_{\nu}(x) d \phi_{\nu}(x)^{2}$ to $g_{0}$ is the same as the stretching the map $f_{0}$ on the split $U_{\nu}$ as described by $[\star]$, the propositions $[\star]$ and [ $\left.\square^{\prime}\right]$ together imply the following

$\left[\star+\square^{\prime}\right]$ Approximately Isometric $C^{1}$-Immersion Proposition. Let $g^{\prime}$ be a continuous positive definite form on $X$, and let $f_{0}: X \rightarrow Y=(Y, \tilde{g})$ be a smooth immersion. If $\operatorname{dim}(Y) \geq \operatorname{dim}(X)+1$, then $f_{0}$ admits a fine $C^{0}$-approximation by $C^{1}$ maps $f_{1}: X \rightarrow Y$, such that the induced metrics $(D f)^{\star}(\tilde{g})$ finely $C^{0}$-approximate $g_{0}+g^{\prime}$.

This proposition, however, is not sufficient for proving the isometric immersion theorem, since it guarantees no control over the $C^{1}$-distances from the approximating maps $f_{1}: X \rightarrow Y$ to $f_{0}: X \rightarrow Y$ which is needed for $C^{1}$-convergence of a consecutive approximations sequence $f_{0}, f_{1}, f_{2}, \ldots$

To regain this control in the general settings of convex hulls, etc., one needs to bring forth the following nowhere flatness assumption on the sets $E_{x}^{\mid}=E_{\underline{D f(x)}}^{\mid} \subset$ $T_{y}(Y)$ that mimics the relevant property of the spheres $S^{q-n}(R)$.

The points $e$ in the convex hull of $E_{x}^{\mid} \subset T_{y}(Y), y=f(x)$ that are close to $E_{x}^{\mid}$are representable by convex combinations of points in $E_{x}^{\mid}$that are close $e$.

In other words,

the convex hull $\operatorname{conv}\left(E^{\bullet}\right) \subset T_{y}(Y)$ of every open subset $E^{\bullet} \subset E_{x}^{\mid}$contains some neighbourhood $V \subset \operatorname{conv}\left(E_{x}^{\mid}\right)$of $E^{\bullet} \subset \operatorname{conv}\left(E_{x}^{\mid}\right)$.

Then an application of $\left[O \sim \Omega\right.$ and $\left[M \mathcal{W}\right.$ ] to (small) open subsets $E^{\bullet} \subset E$ yields a version of $[\perp]$ with a control over $\left\|f_{0}-f\right\|_{C^{1}}$ that, in particular, is sufficient for the proof of the Nash-Kuiper $g_{0}+g^{\prime}$-theorem.

Defence of the Method. What is the point of replacing the half-page direct proof of the Nash-Kuiper isometric $C^{1}$-immersion theorem by several pages of general arguments that do not even make a complete proof?

And the above was not even a derivation of Nash-Kuiper entirely from general principles, since the $\sum \psi_{\nu} d \phi_{\nu}^{2}$-decomposition was borrowed from the original proofs by Nash and by Kuiper,

However a full abstract scheme of the convex integration, that, in particular, digests and incorporates $\sum \psi_{\nu} d \phi_{\nu}^{2}$-decomposition, has been worked out and an abstract Nash-Kuiper $\left[g_{0}+g^{\prime}\right]$-kind of $h$-principle for $C^{1}$-maps $f: X \rightarrow Y$ that satisfy $D f(x) \in E_{x, y}, y=f(x), x \in X$, was proved for those subsets $E_{x, y} \subset$ $\operatorname{hom}\left(T_{x}(X) \rightarrow T_{y}(Y)\right)$ for which a certain partial convex hull is equal to the full (i.e., ordinary) convex hull) (see 2.4 of [35] and also [27] and [70]).

Then the proof of the $C^{1}$-immersion theorem is reduced to a trivial verification of this property for the subset $E=\operatorname{Iso}\left(\mathbb{R}^{n} \rightarrow \mathbb{R}^{q}\right) \subset \operatorname{hom}\left(\mathbb{R}^{n} \rightarrow \mathbb{R}^{q}\right)$.

But just defining "partial convex hulls", formulating the relevant $h$-principle, and listing down the properties of $E=\operatorname{Iso}\left(\mathbb{R}^{n} \rightarrow \mathbb{R}^{q}\right)$ needed for the equality $\operatorname{partconv}(E)=\operatorname{conv}(E)$, even if considered to be quite obvious, takes longer than proving the $C^{1}$-immersion theorem directly.

However, the reason for the length of the proof of the Nash-Kuiper theorem by convex integration is not its inherently mathematical complexity, but rather

with a bound on the number of terms in the sum, was used by Nash in his 1956 paper for construction of isometric $C^{\infty}$-embeddings. 
the necessity of introducing specific terminology adapted to new concepts. Non-

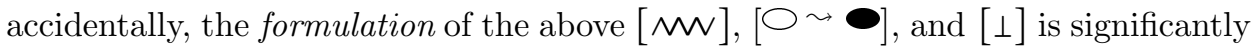
longer than their proofs.

What attracts us in these general "convex concepts" is that once they are accepted and assimilated - with no regard for isometric immersions - all results of the isometric $C^{1}$-immersion theory become plainly obvious, without any reference to their proof, obvious in the same way as, for example, vanishing of the gradient of a smooth function at its maxima points is obvious.

The above justification of convex integration may not be so convincing as far as isometric $C^{1}$-immersions are concerned, but there are classes of maps where convex integration is the only (or at least the simplest known) way of proving the $h$-principle.

For instance (this is a version of the Euclidean short approximation theorem mentioned earlier),

a strictly distance decreasing map between Riemannian manifolds, $f_{0}: X^{n} \rightarrow Y^{q}$, $q>n$, admits a uniform approximation by isometric $C^{1}$-immersions if and only if $f_{0}$ is homotopic to a smooth immersion $X^{n} \rightarrow Y^{q}$.

Another instructive instance where convex integration (trivially!) applies and where no alternative approach is available is in showing that

all parallelisable $n$-manifolds (e.g., all orientable 3-manifolds) with smooth positive volume forms on them admit $n$ linearly independent divergence free (i.e., preserving their volume form) vector fields (see 2.4.3 in [35] and also [27]).

\subsubsection{Open Problems in Convex Integration.}

A. Induced Forms of Degree $p \geq 2$, etc. Application of the convex integration to $C^{1}$-maps $f: X \rightarrow Y$ that induce a given first order structure $g$ on $X$ from $\tilde{g}$ on $Y$ raises several questions, such as the following.

26 What is the weakest hyperregularity (see below) condition on the differentials $D f(x): T_{x}(X) \rightarrow T_{f(x)}(Y)$ that makes convex integration applicable to the equation $(D f)^{\star}(\tilde{g})=g$ ?

27 Given a continuous map $f_{0}: X \rightarrow Y$, when does there exist a continuous family of linear maps $T_{x}(X) \rightarrow T_{y}(Y), y=f_{0}(x)$, that induce $g_{x}$ on the tangent spaces $T_{x}(X)$ from $\tilde{g}_{y}$ on $T_{y}(Y)$ for all $x \in X$ ?

28 What is the homotopy structure of the space of continuous fibrewise linear maps $T(X) \rightarrow T(Y)$ that induce $g_{x}$ from $\tilde{g}_{y}$ on all tangent space $T_{x}(X)$ ?

29 How does the hyperregularity condition on the homomorphisms $T_{x}(X) \rightarrow T_{y}(Y)$ influence this structure?

For example, let $g$ and $\tilde{g}$ be symmetric differential forms of degree $p$.

If $p=2$ and the quadratic form $\tilde{g}$ on $Y$ is non-singular of the type $\left(q_{+}, q_{-}\right)$, $q_{+}+q_{-}=q=\operatorname{dim}(Y)$, then hyperregularity of a linear map $h_{x}: T_{x}(X) \rightarrow T_{y}(Y)$ says, in effect that

(a) $h_{x}$ is $\tilde{g}$-regular which amounts to injective for non-singular $\tilde{g}$;

(b) the type $\left(q_{+}, q_{-}\right)$of $\tilde{g}$ "strictly dominates" $\left(n_{+}, n_{-}\right)=\left(n_{+}, n_{-}\right)_{x}$ of $g_{x}$ (the latter may depend on $x$ for a singular form $g$ ) for all $x \in X$,

$$
q_{+} \geq n-n_{+}+1, \text { and } q_{-} \geq n-n_{-}+1, n=\operatorname{dim}(X)
$$

(where only the inequality $q_{+} \geq n_{+}+1$ is needed for positive definite $g$, i.e., if $\left.n_{-}=n=\operatorname{dim}(X)\right)$. 
Also, the structure of space of the continuous fibrewise injective linear maps $T(X) \rightarrow T(Y)$ that induce $g_{x}$ from $\tilde{g}_{y}$ on all tangent spaces $T_{x}(X)$ is fairly well understood in this case, especially if $g$ is non-singular or, more generally, if the type $\left(n_{+}, n_{-}\right)_{x}$ does not depend on $x$, e.g., $g$ is identically zero.

Thus, for instance, if $X$ is homeomorphic to $\mathbb{R}^{n}$ and the type $\left(n_{+}, n_{-}\right)_{x}$ of $g$ is constant and is strictly dominated by that of $\tilde{g}$ (as in (b)), then

every continous map $f_{0}: X \rightarrow Y$ can be $C^{0}$-approximated by $C^{1}$-maps $f: X \rightarrow Y$ that induce $g$ from $\tilde{g}$.

But everything becomes more complicated for forms of degrees $p \geq 3$, where the concepts of $\tilde{g}$-regularity and hyperregularity conditions needed for present day convex integration techniques to work becomes rather heavy and, possibly, unnecessary in their present form (see 2.4.9 in 35]).

Besides, the solution of the algebraic and the homotopy theoretic problems to which the $h$-principle reduces the solvability of the equation $(D f)^{\star}(\tilde{g})=g$ remains problematic.

On the one hand, one does have some existence results for "sufficiently nondegenerate" $\tilde{g}$.

For instance, let $\tilde{g}$ be a diagonal form of degree $p$ on a smooth $q$-dimensional manifold $Y$,

$$
\tilde{g}=\sum_{i=1}^{q_{+}} l_{i}^{p}-\sum_{j=1}^{q_{-}} l_{j}^{p},
$$

where $q_{+}+q_{-}=q=\operatorname{dim}(Y)$ and where $\left\{l_{i}, l_{j}\right\}$ is a $q$-tuple of everywhere linearly independent degree 1 differential forms on $Y$, e.g., $\tilde{g}=\sum_{i=1}^{q_{+}} d y_{i}^{p}-\sum_{j=1}^{q_{-}} d y_{j}^{p}$ on $\mathbb{R}^{q_{+}+q_{-}}$. One knows (see 2.4.9 in [35]) that

if $q_{+}, q_{-} \geq s_{n+1, p-1}+n$ for $n=\operatorname{dim} X$ and $s_{n, p}=\frac{n(n+1) \cdots(n+p-1)}{p !} 31$ then an arbitrary continuous map $X \rightarrow Y$ admits a fine $C^{0}$-approximation by $C^{1}$-maps $X \rightarrow Y$ that induce a given form $g$ of degree $p$ on $X$ from $\tilde{g}$ on $Y$.

\section{QUESTIONS}

Can one improve this estimate on $q_{ \pm}$?

For example,

what is the minimal $q$ such that every 3 -form $g$ on an $n$-manifold $X$ decomposes as $g=\sum_{1}^{q} l_{i}^{3}$ for some $C^{1}$-smooth exact linear forms $l_{i}$ on $X$ ? What are the classes of non-symmetric differential forms $g$ and $\tilde{g}$ where the convex integration applies.33

What are convex cone problems among those indicated and/or hinted at in section 2.2 that are amenable to convex integration?

B. Isometric Immersion with External Constraints. Below is an instance of a class of maps where convex integration fails but more direct constructions by Nash and by Kuiper work fine.

Let $\mathcal{F}_{+m}$ be a sheaf of $C^{1}$-maps from open subsets $U \subset X \times \mathbb{R}^{m}$ to $Y$, and let $\mathcal{F}$ be a sheaf of maps $\mathbb{R}^{n}$ to $Y$ with the following two properties.

\footnotetext{
${ }^{31}$ This $s_{n, p}$ is equal to the number of differential equations corresponding to the relation $(D f)^{\star}(\tilde{g})=g$.

${ }^{32}$ If you drop "exact" this becomes a problem in algebraic topology; yet, no satisifactory answer seems available.

${ }^{33}$ Some cases were treated by Mahuya Datta with coauthors, where the references can be found on http://www.isical.ac.in/ mahuya/publications.html.
} 
1. If a map $f: U \rightarrow Y, U \subset X$, is contained in $\mathcal{F}(U)$, where $U$ is "sufficiently small" 34 then $f$ extends from $U=U \times 0 \subset U \times \mathbb{R}^{m}$ to a map $f_{+}: U \times \mathbb{R}^{m} \rightarrow Y$ in $\mathcal{F}_{+m}\left(U \times \mathbb{R}^{m}\right)$, such that the composition of $f_{+}$with the graphs $\Gamma_{\phi}: U \rightarrow U \times \mathbb{R}^{m}$ of $C^{\infty}$ maps $\phi: U \rightarrow \mathbb{R}^{m}$ is contained in $\mathcal{F}(U)$.

2. The space of $C^{1}$-maps $f: X \rightarrow Y$ from $\mathcal{F}\left(X \times \mathbb{R}^{m}\right)$ is closed in the $C^{1}$-topology.

$\left[g_{0}+g^{\prime}\right]$-Approximation in $\mathcal{F}$. Let $f_{0}: X \rightarrow Y$ be a $C^{1}$-immersion contained in $\mathcal{F}(X)$, and let $g^{\prime}$ and $\tilde{g}$ be continuous positive definite quadratic forms on $X$ and on $Y$, respectively. Then

if $m \geq 1$, then $f$ admits an arbitrarily fine $C^{0}$-approximations by $C^{1}$-maps $f^{\prime}$ : $X \rightarrow Y$ such that $f^{\prime} \in \mathcal{F}(X)$ and $\left(D f^{\prime}\right)^{\star}(\tilde{g})=\left(D f_{0}\right)^{\star}(\tilde{g})+g^{\prime}$.

Discouraging Remark. The above proposition tells us something new compared to the Nash-Kuiper theorem only if $f_{0} \in \mathcal{F}(X)$ does not come from the restriction of some $f_{+} \in \mathcal{F}\left(X \times \mathbb{R}^{m}\right)$ to $X=X \times 0 \subset X \times \mathbb{R}^{m}$.

Example. Let $Y=(Y, \omega)$ be a symplectic manifold, and let $\mathcal{F}$ be the sheaf of $\omega$-isotropic maps $f: U \rightarrow Y, U \subset X$, i.e., $(D f)^{\star}(\omega)=0$.

$\left[g_{0}+g^{\prime}\right]$-Isotropic Approximation. Let $g^{\prime}$ and $\tilde{g}$ be continuous positive definite quadratic forms on $X$ and $Y$, respectively, and let $f_{0}: X \rightarrow Y$ be an $\omega$-isotropic $C^{1}$-immersion. Then the above shows that

if $\operatorname{dim}(Y) \geq 2 \operatorname{dim}(X)+2$, then $f_{0}$ admits arbitrarily fine $C^{0}$-approximations by $\omega$-isotropic $C^{1}$-immersions $f^{\prime}: X \rightarrow Y$ such that $\left(D f^{\prime}\right)^{\star}(\tilde{g})=\left(D f_{0}\right)^{\star}(\tilde{g})+g^{\prime}$.

(If $\operatorname{dim}(Y) \geq 2 \operatorname{dim}(X)+2$ and the form $\tilde{g}$ is quasi-Hermitian, this follows from [19].)

34 Question. Can one generalise convex integration to a point where it would accomadate the above $\left[g_{0}+g^{\prime}\right]$-approximation along with symplectic and contact isometric embedding theorems from [17] and [19]?

$\left[g_{0}+g^{\prime}\right]$-Questions. The above proof of the $\left[g_{0}+g^{\prime}\right]$-approximation fails for Lagrangian immersions, i.e., when $\operatorname{dim}(Y)=2 \operatorname{dim} X$, but

the approximation statement itself, probably, remains true.

More generally, let $Y$ be a smooth manifold, and let $\mathcal{D}$ be a pseudo-group of diffeomorphisms acting on $Y$. For instance, $Y$ may be symplectic and $\mathcal{D}$ may consist of Hamiltonian diffeomorphisms.

Let $\operatorname{Gr}_{n}(Y)$ denote the space of tangent $n$ planes $T_{y}^{\prime} \subset T_{y}(Y), y \in Y$, and let $X^{\prime} \subset \mathrm{Gr}_{n}(Y)$ be a closed subset the projection of which to $Y$ is one-to-one. For instance, $\tilde{X}$ may be equal to the tangential lift of a $C^{1}$-submanifold $X=X^{n} \subset Y$.

Let $\tilde{g}_{0}$ be a continuous family of positive definite quadratic forms induced from $\tilde{g}$ on the linear $n$-spaces $T_{y}^{\prime} \in X^{\prime}$, and let $g^{\prime}$ be another continuous family of positive definite forms on these $T_{y}^{\prime}$.

36 Under what circumstances does there exist a diffeomorphism $\delta^{\prime} \in \mathcal{D}$, such that the form on $X^{\prime}$ induced from $\tilde{g}$ by the differential of $\delta^{\prime}$ satisfies

$$
\left(D \delta^{\prime}\right)^{\star}(\tilde{g})=\tilde{g}_{0}+g^{\prime} ?
$$

\footnotetext{
${ }^{34}$ This means that there exists a covering of $X$ by open subsets $U_{i}$ (this covering should be independent of $f$ ), such that $U$ is contained in some of these $U_{i}$.
} 
C. Hölder $C^{1, \alpha}$-Maps. Convex integration, applied to the first order systems of differential equations, such as $(D f)^{\star}(\tilde{g})=g$, can, in principle, deliver solutions $f$ that are more regular than $C^{1}$, e.g., Hölder $C^{1, \alpha}$ for some $0<\alpha<1$.

The first result of this kind goes back to Borisov (7 1965, 8, 2004) and was then developed by Conti, De Lellis, and Székelyhidi [15] (2012), where these authors refine Kuiper's stretching argument and construct, in particular,

local isometric $C^{1, \alpha}$-immersions of $C^{\beta}$-smooth Riemannain manifolds $X^{n}$ to $\mathbb{R}^{q}$, $q>n$, for $\alpha<\min \left(\frac{1}{1+(n(n+1)}, \beta / 2\right)$.

Also, according to Källen [52] (1978),

$C^{l, \beta}$-manifolds, $l=0,1,0<\beta<1$, admit isometric $C^{1, \alpha}$-embeddings to $\mathbb{R}^{q}$ for $q=3(n+1)\left(n^{2}+n+2\right)+2 n$.

I must admit I have not studied the arguments by Borisov, Källen, and Conti-De Lellis-Székelyhidi, and I do not know how to set them into the general framework of convex integration, but, I guess, their methods may be applied to other structure inducing equations we meet in the present paper. In any case, the following questions remains open.

For which $n$ and $q=q(n, \alpha)$, for a given $0<\alpha<1$, can strictly distance decreasing immersions between $C^{2}$-smooth Riemannian manifolds, $f_{0}: X^{n} \rightarrow Y^{q}$, be approximated by isometric $C^{1, \alpha}$-smooth immersions?

Is there a significant mutual dependence between $q$ in the range $n+1 \leq s_{n}-1$ and $\alpha$ ?

Are there (local) $C^{1, \alpha}$-immersions of general sufficiently smooth metrics to $\mathbb{R}^{q}$ for all $\alpha<1 / 2$ and some (most?) $q<s_{n}$ ?

More generally, let

$$
E=\bigcup_{x, y} E_{x, y} \subset H=\operatorname{hom}(T(X) \rightarrow T(Y)
$$

be a subset that is amenable to convex integration, e.g., the $C^{\perp}$-approximation holds for maps $f: X \rightarrow Y$ that satisfy $D f(x) \in E_{x, f(x)}$ with respect to some splitting $X=\underline{X} \times \mathbb{R}$.

What are the extra conditions, depending on a given $0<\alpha<1$, that one needs to impose on $E$ in order to guarantee the existence of $C^{1, \alpha}$-maps $f: X \rightarrow Y$ that satisfy $D f(x) \in E_{x, f(x)}$ for all $x \in X$ ?

(An apparently relevant condition is a quantified non-flatness of $E$.)

D. Differential Equations of Orders $\geq 2$. Convex integration applies to $C^{r}-$ solutions of differential equations of all orders $r \geq 1$, e.g., to those characterising maps inducing $r$ th order structures, but definite results are known only in a few specific examples, such as construction of $C^{2}$-maps $X \rightarrow \mathbb{R}^{q}$ with prescribed (extrinsic) curvatures. But even that was achieved by means of a direct Nash-style construction rather than with the help of abstract convex integration (see 3.1.5 in 35]).

Thus, besides extensions of the problems mentioned in this section to $r \geq 2$, one needs an understanding of possible examples where convex integration may be applied.

\subsection{Solving Differential Equations by Algebraic Formulas.}

There exists no universal formula $f=\Xi(g)$ that would express the components $f_{1}, f_{2}, \ldots, f_{q}: X \rightarrow \mathbb{R}$ of a map $f: X \rightarrow \mathbb{R}^{q}$ as polynomials (or general algebraic 
functions) in the components of the Riemannian metric $g$, that would make the map $f$ isometric, i.e., such that $(D f)^{\star}\left(\sum_{j} d y_{j}^{2}\right)=g$, or, equivalently, such that

the composition of the two (non-linear) differential operators

$$
g \mapsto f=\Xi(g) \text { followed by } f \mapsto g=(D f)^{\star}\left(\sum_{j} d y_{j}^{2}\right)
$$

is equal to the identity operator $g \mapsto g$.

Amazingly, this can be done, and quite easily at that, if instead of the Euclidean $\sum_{j} d y_{j}^{2}$ we use an indefinite form $\sum_{j} d y_{j}^{2}-\sum_{k} d y_{k}^{2}$ as follows.

\subsubsection{Algebraic Immersions to Pseudo-Euclidean Spaces.}

To make the story $100 \%$ easy, assume that $X$ is diffeomorphic to $\mathbb{R}^{n}$ and write the isometric immersion equation for maps $f:(X, g) \rightarrow \mathbb{R}^{q}$ in local coordinates as

$$
\left\langle\partial_{i} f, \partial_{j} f\right\rangle=g_{i j}
$$

where $\partial_{i} f(x)=\frac{\partial f(x)}{\partial x_{i}} \in \mathbb{R}^{q}$ and $g_{i j}=g_{i j}(x)$ are the components of $g$.

Let $\underline{f}: X \rightarrow \mathbb{R}^{q}, q=s_{n}+n, s_{n}=\frac{n(n+1)}{2}$ be a free $C^{2}$-smooth map that is such that its first and second partial derivatives $\partial_{i} \underline{f}(x), \partial_{i j}^{2} f(x) \in \mathbb{R}^{q}$ are linearly independent at all $x \in X$. For instance, one may take $\underline{f}=\left(x_{1}, \ldots, x_{n}, x_{1}^{2}, x_{1} x_{2}, x_{1} x_{3}, \ldots\right.$, $\left.x_{n-1} x_{n}, x_{n}^{2}\right)$.

Let $\underline{g}$ be the metric on $X$ induced by $\underline{f}$, let $f^{\prime}: X \rightarrow \mathbb{R}^{q}$ be another smooth map, and observe that

- the metric $\underline{g}^{\prime}$ induced by $\underline{f}+f^{\prime}$ equals

$$
\underline{g}_{i j}^{\prime}=\left\langle\partial_{i}\left(\underline{f}+f^{\prime}\right), \partial_{j}\left(\underline{f}+f^{\prime}\right)\right\rangle=\underline{g}_{i j}+2\left\langle\partial_{i} f^{\prime}, \partial_{j} \underline{f}\right\rangle+g_{i j}^{\prime},
$$

where $g_{i j}^{\prime}=\left\langle\partial_{i} f^{\prime}(x), \partial_{j} f^{\prime}(x)\right\rangle$ is the quadratic form induced by $f^{\prime}$.

•- The partial derivatives of the scalar products $\phi_{i}^{\prime}=\left\langle f^{\prime}, \partial_{i} \underline{f}\right\rangle$ satisfy

$$
\partial_{j} \phi_{i}^{\prime}=\left\langle\partial_{i} f^{\prime}, \partial_{j} \underline{f}\right\rangle+\left\langle f^{\prime}, \partial_{i j}^{2} \underline{f}\right\rangle .
$$

This allows us to rewrite $\bullet$ as

$$
\begin{gathered}
\underline{g}_{i j}^{\prime}=\underline{g}_{i j}+\psi_{i j}^{\prime}-2\left\langle f^{\prime}, \partial_{i j}^{2} \underline{f}\right\rangle+g_{i j}^{\prime}, \\
\psi_{i j}^{\prime}=\partial_{j} \phi_{i}^{\prime}+\partial_{i} \phi_{j}^{\prime} .
\end{gathered}
$$

Finally, given an arbitrary quadratic differential form $g$ on $X$, the freedom of $f$ guarantees the existence of an $f^{\prime}=f^{\prime}(g)$ (actually of a unique one) such that

$$
2\left\langle f^{\prime}, \partial_{i j}^{2}\right\rangle=-g_{i j}+\underline{g}_{i j}+\psi_{i j}^{\prime}
$$

hence, the metric $\underline{g}_{i j}^{\prime}$ induced by $\underline{f}+f^{\prime}: X \rightarrow \mathbb{R}^{q}$ is

$$
\underline{g}^{\prime}=g+g^{\prime}
$$

and the metric induced by $F=\left(\underline{f}+f^{\prime}, f^{\prime}\right): X \rightarrow \mathbb{R}^{2 q}=\mathbb{R}^{q_{+}} \oplus \mathbb{R}^{q_{-}}$from the form

$$
\sum_{j=1}^{q} d y_{j}^{2}-\sum_{k=1}^{q} d y_{k}^{2} \text { for } q_{+}=q_{-}=q=\frac{n(n+1)}{2}+n,
$$

is equal to $g$. QED 
Two Technical Remarks. (a) The above scheme describes all generic isometric maps $F:(X, g) \rightarrow \mathbb{R}^{q_{+}} \oplus \mathbb{R}^{q_{-}}$, namely those for which the projections to $\mathbb{R}^{q_{+}}$are free, where these $F$ depend on $s_{n}+2 n$ arbitrary functions, namely the $s_{n}+n$-components of $f$ and $\phi_{i}^{\prime}$.

(b) The above trivially generalises to all $n$-manifolds $X=X^{n}$, where, a priori, one needs $q=s_{n}+2 n$ to ensure free immersions $f \rightarrow \mathbb{R}^{q}$. But it seems not hard, however, to do it with generic $C^{\infty}$-maps $X \rightarrow \mathbb{R}^{\bar{q}}$ for $q=s_{n}+n$ with some care taken in solving the system of linear equations $2\left\langle f^{\prime}, \partial_{i j}^{2}\right\rangle=? ? ?$ at the codimension one subvariety where this system becomes singular. Moreover, it is plausible that this works already for $q=s_{n}+1$ (see section 3.6.2)?

\section{Questions}

Are isometric immersions $X^{n} \rightarrow \mathbb{R}^{q_{+}} \oplus \mathbb{R}^{q_{-}}$by universal algebraic formulas possible for $\min \left(q_{+}, q_{-}\right) \leq s_{n}$ ?

In fact, there is neither construction nor obstruction in $\operatorname{sight}$ if $\min \left(q_{+}, q_{-}\right) \geq 2 n$ and $q_{+}+q_{-} \geq s_{n}$.

(The existence of an isometric $C^{\infty}$-immersion $(X, g) \rightarrow \mathbb{R}^{q_{+}} \oplus \mathbb{R}^{q_{-}}$is known for $\min \left(q_{+}, q_{-}\right) \geq 2 n$ and $q_{+}+q_{-} \geq s_{n}+3 n$; see 3.3 in [35.)

What are other instances of algebraic pseudo-Riemannian manifolds $Y$, besides $\mathbb{R}^{q_{+}} \oplus \mathbb{R}^{q_{-}}$, such that general manifolds $\left(X^{n}, g\right)$ admit algebraic isometric immersions to these $Y$ ?

Do spaces of (regular in some sense, e.g., free) algebraic isometric immersions $X \rightarrow Y$ ever satisfy some $h$-principle?

Historical Remarks. Janet-Burstin's argument was adapted by A. Friedman [28] (1961), to

local isometric embeddings of pseudo-Riemannian $C^{a n}$-manifolds $X$ of type $\left(n_{+}, n_{-}\right), n_{+}+n_{-}=n=\operatorname{dim}(X)$ to the pseudo-Euclidean space $\mathbb{R}^{q_{+}, q_{-}}$with $q_{ \pm} \geq n_{ \pm}$ and $q_{+}+q_{-}=q \geq s_{n}$.

The $C^{\infty}$-case remains problematic; but it is not impossible, for instance, that the existence of local isometric embeddings of Riemannian $C^{\infty}$-manifolds to a pseudoEuclidean space $\left(q_{+}, q_{-}>0\right)$ may be easier than such an embedding to the Euclidean $\left(q_{-}=0\right)$ space of the same dimension $q$.

It was observed by R. E. Greene (31 1969, 32 1970) and C. J. S. Clarke (14 1970) that if a manifold $X$ admits a proper smooth immersion to $\mathbb{R}^{q_{+}}$, e.g., if $q_{+} \geq 2 \operatorname{dim}(X)$, then there exists a proper embedding, say $f_{+}: X \rightarrow \mathbb{R}^{q_{+}}$, that induces an arbitrary large form $g_{+}$on $X$.

(Such embeddings may be obtained with obvious self-maps $\mathbb{R}^{q_{+}} \rightarrow \mathbb{R}^{q_{+}}$that arbitrarily strongly stretch the Euclidean space $\mathbb{R}^{q_{+}}$.)

Therefore, an arbitrary quadratic differential $C^{\infty}$-form $g$ on $X$ decomposes as $g_{+}-g_{-}$where $g_{ \pm}$are Riemannian $C^{\infty}$-metrics and where $g_{+}$is induced by a $C^{\infty}{ }_{-}$ embedding $f_{+}: X \rightarrow \mathbb{R}^{q_{+}}$.

Thus, Greene and Clarke conclude that

Riemannian isometric $C^{\infty}$-immersions $\left(X, g_{-}\right) \rightarrow \mathbb{R}^{q_{-}}$yield proper pseudo-Riemannain isometric embeddings $(X, g): \mathbb{R}^{q_{+}, q_{-}}$for $q_{+}=2 n$ and all quadratic differential forms $g$ on $X$.

Also, Greene observes that if $X$ is compact, then Nash's perturbation/implicit function theorem, applied to free isotropic (i.e., inducing zero form) immersions 
$F_{0}: X \rightarrow \mathbb{R}^{q_{+}, q_{-}}$, yields isometric $C^{\infty}$-immersions $F_{\varepsilon}:(X, \varepsilon g) \rightarrow \mathbb{R}^{q_{+}, q_{-}}$for all $C^{\infty}{ }_{-}$ smooth $g$ and small $\varepsilon=\varepsilon(g)>0$.

Thus, departing from a (generic) free embedding $f_{0}: X \rightarrow \mathbb{R}^{q}, q=s_{n}+2 n$, Greene obtains isometric $C^{\infty}$-embedding $F=\varepsilon^{-\frac{1}{2}} F_{\varepsilon}:(X, \varepsilon g) \rightarrow \mathbb{R}^{q_{+}, q_{-}}, q_{ \pm}=q$, for $F_{\varepsilon}$ being the above perturbation of

$$
F_{0}=\left(f_{0}, f_{0}\right): X \rightarrow\left(\mathbb{R}^{q_{+}, q_{-}}, \sum_{j=1}^{q} d y_{j}^{2}-\sum_{k=1}^{q} d y_{k}^{2}\right) .
$$

Here, the perturbation theorem may be applied directly to $f_{0}$ and, according to Greene, the case of non-compact manifolds follows, albeit with a larger $q_{ \pm}$, namely, for $q_{ \pm}=(2 n+l)(2 n+6)$.

(There are many papers on isometric embeddings in physics journals where some references may be found in [29, [57, 40. Unfortunately, most of these papers are virtually non-existent, since they are not freely accessible on the web: they cannot be read and referred to.)

\subsubsection{Algebraic Solution of Linear PDE and Infinitesimal Inversion of Non-Linear} Differential Operators.

Let $L$ be a linear differential operator, say with $C^{\infty}$-coefficients, of order $r=r_{L}$ that sends $q$-tuples $f$ of functions on $\mathbb{R}^{n}$ to $s$-tuples $g$, and let us address the following questions:

Does $L: f \mapsto g$ admit a left inverse differential operator of certain order $r=r_{M}$, say $M: g \mapsto f$, i.e., such that $M \circ L(f)=f$ for all $f$ ?

Does $L: f \mapsto g$ admit such a right inverse $M$, i.e., $L \circ M(g)=g$ for all $g$ ?

It may feel counterintuitive, but

a differential operator $L$ with generic coefficients admits a left inverse if $q<s$ and a right inverse if $q>s$.

Justification. The relation

$$
M \circ L=i d
$$

is representable by a system of equations on the coefficients of $M$ that are linear algebraic equations, the coefficients of which are served by partial derivatives of the coefficients of $L$.

The number $u_{M}$ of unknowns, which are coefficients of $M$ in these equations, is $u_{M}=q s P_{n}\left(r_{M}\right)$ for some universal polynomial $P_{n}$, while the number of equations $e_{L, M}$ equals $q^{2} P_{n}\left(r_{L}+r_{M}\right)$.

If our unknown $M$ is selected among operators of a sufficiently high order $r_{M}$ and we are free to try $M$ of any order we like - then the inequality $s>q$ imples that $u_{M}>e_{L, M}$, which makes the algebraic system underdetermined. Therefore, it is plausible (and easily provable, see 2.3.8 in [35]) that

if $s>q$, then the system of equations corresponding to $M \circ L=i d$ is solvable in $M$, for generic operators $L$.

Corollary. A generic overdetermined system of linear differential equations $L(f)=g$ may have at most a single solution $f$.

Now we turn to the right inversion relation

$$
L \circ M=i d,
$$

which expands to a system of linear differential equations of order $r_{L}$ on the coefficients of $M$. 
We are going to transform this differential system to an algebraic one by means of a

universal differential operator(s) $I$, such that $I \circ I=i d$.

The operators $I$ we use here act on coefficients of differential operators by conjugation, that is taking formal adjoints, denoted $L \rightarrow L^{I}, M \rightarrow M^{I}$, where $L^{I}: g \mapsto f$, $M^{I}: f \mapsto g$, and

$$
(L \circ M)^{I}=M^{I} \circ L^{I} .
$$

This reduces the right inversion problem $L \circ M=i d$ to the left one for $M^{I} \circ L^{I}=i d$, since

$$
i d^{I}=i d,\left(M^{I}\right)^{I}=M, \text { and }\left((L \circ M)^{I}\right)^{I}=L \circ M .
$$

Thus, assuming solvability of the "left problem", we conclude that

if $q>s$, then the system of equations corresponding to $L \circ M=i d$ is solvable in $M$, for generic operators $L$.

Corollary. Generic underdetermined systems of linear differential equations $L(f)=g$ are algebraically solvable, i.e., by applying differential operators $M=M_{L}$ to $g$.

\section{Questions}

What is the structure of the group $\mathcal{D}_{n, q}$ of invertible elements in the semigroup of differential operators that act on $q$ tuples on functions $f$ in $n$ variables?

Is $\mathcal{D}_{n, q}$ generated by some kind of "elementary transformations" such as $\left(f_{1}, f_{2}\right) \mapsto$ $\left(f_{1}, f_{2}+\partial_{i} f_{1}\right)$.

(The conjugation operators $I$ as well as automorphisms of supermanifolds are of this nature.)

InfINITESIMAL INVERTIBILITY. A non-linear differential operator $\mathcal{D}$ between spaces of functions/maps, say $\mathcal{D}: \mathcal{F} \rightarrow \mathcal{G}$ is called infinitesimally invertible at functions $f_{0}$ from some open subset $\mathcal{F}_{0} \subset \mathcal{F}$ if the linearised operators between the respective tangent spaces,

$$
L_{\mathcal{D}, f_{0}}: T_{f_{0}}(\mathcal{F}) \rightarrow T_{\mathcal{F}\left(f_{0}\right)}(\mathcal{G}),
$$

are algebraically invertible, namely, there exist differential operators

$$
M=M_{f_{0}}: T_{\mathcal{D}\left(f_{0}\right)}(\mathcal{G}) \rightarrow T_{f_{0}}(\mathcal{F}), f_{0} \in \mathcal{F}_{0},
$$

such that

$$
\left(L_{\mathcal{D}, f_{0}} \circ M_{f_{0}}\right)\left(g^{\prime}\right)=g^{\prime}, g^{\prime} \in T_{\mathcal{F}\left(f_{0}\right)}(\mathcal{G}),
$$

where, moreover, $M_{f_{0}}\left(g^{\prime}\right)$ is a differential operator in both variables, $f_{0} \in \mathcal{F}_{0}$ as well as in $g^{\prime} \in T_{\mathcal{F}\left(f_{0}\right)}(\mathcal{G})$.

Example. Let $Y=(Y, \tilde{g})$ be a pseudo-Riemannian manifold, and $\mathcal{D}=\mathcal{D}_{\tilde{g}}$ sends maps $f: X \rightarrow Y$ to the induced forms $g$ on $X$,

$$
\mathcal{D}(f)=g=(D f)^{\star}(\tilde{g}) .
$$

The operator $\mathcal{D}: f \mapsto(D f)^{\star}(\tilde{g})$ is infinitesimally invertible at free maps $f_{0}: X \rightarrow Y$.

In fact, we have already met the linearised system $L_{f_{0}}\left(f^{\prime}\right)=g^{\prime}$ in the previous section, which now reads

$$
\left\langle\partial_{i} f^{\prime}, \partial_{j} f_{0}\right\rangle_{\tilde{g}}+\left\langle\partial_{j} f^{\prime}, \partial_{i} f_{0}\right\rangle_{\tilde{g}}=g_{i j}^{\prime} .
$$

We augment this with the equation

$$
\left\langle f^{\prime}, \partial_{i} f_{0}\right\rangle=0
$$


and, by covariantly differentiating these, obtain the relation

$$
\left\langle\partial_{i} f^{\prime}, \partial_{j} f_{0}\right\rangle_{\tilde{g}}+\left\langle\partial_{j} f^{\prime}, \partial_{i} f_{0}\right\rangle_{\tilde{g}}=-2\left\langle f^{\prime}, \partial_{i j}^{2} f_{0},\right\rangle .
$$

Thus $[L]$ is reduced to the system of linear algebraic equations

$$
\begin{aligned}
\left\langle f^{\prime}, \partial_{i} f_{0}\right\rangle & =0, \\
2\left\langle f,^{\prime} \partial_{i j}^{2} f_{0},\right\rangle & =-g_{i j},
\end{aligned}
$$

which, by the definition of freedom (see section 2.4), is non-singular; hence, solvable.

Pseudo-Historical Remark. Infinitesimal invertibility of the metric inducing operators $\mathcal{D}_{\tilde{g}}$ at free maps is a little $\operatorname{cog}$ in the algebra of reduction of the isometric immersion equations to the Cauchy-Kovalevskaia form by Janet and Burstin. But apparently nobody took it seriously prior to the 1956 paper by Nash, who combined it with his perturbation/implicit function theorem and thus has proved that if $X$ is compact, then

$\mathcal{D}_{\tilde{g}}$ is an open map from the space of free $C^{\infty}$-maps $f: X \rightarrow Y=(Y, \tilde{g})$ to the space of $C^{\infty}$-smooth quadratic differential forms on $X$.

CONJECTURE. If $q=\operatorname{dim}(Y)>s_{n}=\frac{n(n+1)}{2}, n=\operatorname{dim}(X)$, then the operator $\mathcal{D}_{\tilde{g}}$ is infinitesimally invertible on an open dense susbset $\mathcal{F}_{0}$ in the space of $C^{\infty}$-maps $f: X \rightarrow Y$.

If true, the operator $\mathcal{D}_{\tilde{g}}$ will be open in the $C^{\infty}$-topology on this $\mathcal{F}_{0}$, by Nash's perturbation theorem. But since the order of the (potential) differential operator $M$ that would invert $L$ must increase with $n$, Nash's theorem would apply to $C^{r}$ perturbations only for $r=r(n) \rightarrow \infty$ as $n \rightarrow \infty$.

At the present moment, there is convincing (97\%) evidence for this if $q \geq$ $s_{n}+n-\sqrt{n / 2}$ (see 2.3.8 in 35]) that was verified (100\%) for $n=2$ in [20] and futher studied for $n \geq 2$ in $[23]$.

Besides, it is known that the operator $f \mapsto(D f)^{\star}(\tilde{g})$ on maps $X \rightarrow(Y, \tilde{g})$ is infinitesimally invertible for forms $\tilde{g}$ of degree $p>2$ at "sufficiently non-degenerate" maps $f_{0}: X \rightarrow Y$ that can be called free relative to $\tilde{g}$.

Also similar relative freedom ensures infinitesimal invertibility of the operators $f \mapsto(D f)^{\star}(\tilde{g})$ for certain non-symmetric differential forms $\tilde{g}$ (see 3.4.1 in [35]).

But easily verifiable criteria for this freedom are yet to be explicitly worked out, and, which is more difficult, freedom at generic maps is expected in most (all?) cases where the structure inducing operator is underdetermined.

\subsubsection{Algebraically Solvable and Unsolvable Polynomial PDEs.}

The existence of algebraic isometric immersions to pseudo-Euclidean spaces from section 3.6.1 can be also derived from the identity

$$
y(d z)^{2}=\frac{1}{2}\left((d(z y+z))^{2}-(d z)^{2}-(d(z y))^{2}-\left(d\left(z^{2}+y\right)\right)^{2}+\left(d\left(z_{2}\right)\right)^{2}+(d y)^{2}\right)
$$

that defines a polynomial isometric embedding

$$
\left(\mathbb{R}^{2}, y(d z)^{2}\right) \rightarrow \mathbb{R}^{3_{+}, 3_{-}} .
$$

Since an arbitrary form $\sum g_{i j} d x_{i} d x_{j}$ on $X$ is tautologically induced from the universal quadratic form $\sum y_{i j} d z_{i} d z_{j}$ on the squared cotangent bundle of $X$ and since

$$
d z_{i} d z_{j}=\frac{1}{4}\left(\left(d\left(z_{i}+z_{j}\right)\right)^{2}-\left(d\left(z_{i}-z_{j}\right)\right)^{2}\right)
$$


the embedding $\left(\mathbb{R}^{2}, y(d z)^{2}\right) \rightarrow \mathbb{R}^{3_{+}, 3_{-}}$yields a polynomial isometric embedding

$$
\left(X=\mathbb{R}^{n}, \sum g_{i j} d x_{i} d x_{j}\right) \rightarrow \mathbb{R}^{q_{+}, q_{-}} \text {for } q_{ \pm}=6 s_{n}-3 n=3 n^{2} .
$$

But, contrary to what I stated in [34], it seems unlikely that the form $y(d z)^{p}$ with $p>2$ decomposes as

$$
y(d z)^{p}=\sum_{k=1}^{l}\left(d f_{k}(y, z)\right)^{p}
$$

with polynomials $f_{k}$.

\section{QUESTIONS}

What are, in general, non-linear differential operators that admit right (left in the overdetermined case) inversions by differential operators?

How representative in these respects are contact transformations, e.g., normal equidistant moves of hypersurfaces in Riemannian manifolds, such as the geometric Legendre transform?

What is the minimal $l$ needed for a decomposition $y(d z)^{p}=\sum_{k=1}^{l}\left(d f_{k}(y, z)\right)^{2}$ with $C^{\infty}$ - or with $C^{a n}$-functions $f_{k}$ ?

Does such a decomposition exist with entire holomorphic functions for $y(d z)^{p}$ on $\mathbb{C}^{2}$ ?

Is there a meaningful theory for structure inducing holomorphic maps from Stein manifolds, e.g., from $\mathbb{C}^{n}$, to simple (Oka?) spaces such as complex Euclidean spaces $\mathbb{C}^{q}$ with translation invariant tensorial structures:35

Are there working criteria for the existence and also for the non-existence of holomorphic solutions of general underdetermined polynomial differential equations?

Is it true, for instance, that generic systems of $s$ such equations of degree $p$ in $q$ unknown functions have no entire holomorphic solutions if $p \geq p_{0}(s, q)$ ?

(If $p=0$, this goes as the Kobayashi conjecture; see [74] and references therein.)

What are systems of algebraic PDEs in $n$-variables solutions of which algebraically reduce to solutions of auxiliary PDEs in $m$-variables for some $m<n$ ?

An instance of this is the above reduction of decompositions $g=\sum_{j}\left(d f_{j}\right)^{p}$ on $n$-dimensional manifolds to such a decomposition of a single form $y(d z)^{p}$ on the two-plane $\mathbb{R}^{2}$ and this will be reduced to a solution of $O D E s$, i.e., of a differential equation in a single variable (see [34]).

But probably,

there is no such kind of reduction with positivity constraints: isometric immersions of general Riemannian manifolds $(X, g)$ to Euclidean spaces are unlikely to be algebraically expressible by means of $\Phi(\mathrm{g})$, for solutions $\Phi$ of particular differential equations.

\subsection{Nash Implicit Function Theorem.}

The Nash proof of the isometric $C^{r}$-embedding theorem for $r>2$ of compact manifolds $X$ to Euclidean spaces is composed of two ingredients.

1. Geometry. The (convex, see section 2.2) (sub)cone Cone $\mathrm{Euc}^{r}$ of $C^{r}$-metrics on $X$ that are induced by $C^{r}$-maps to Euclidean spaces is dense in the space of all $C^{r}$-metrics.

2. Analysis. The subcone Cone ${ }_{\text {Euc }}^{r}$ contains a non-empty subset that is open in the space of all $C^{r}$-metrics on $X$.

\footnotetext{
${ }^{35}$ I suggested something at the end of my article on the Oka principle [36], but apparently, I was mistaken.
} 
Consequently - Nash argues - since the sums of inducible metrics are inducible, all $C^{r}$-metrics on compact manifolds can be induced by $C^{r}$-embeddings $X \rightarrow \mathbb{R}^{q}$, where the specific value of $q$ obtained by Nash by quantifying this argument is as follows 36

$$
q_{\mathrm{Nash}}=q_{1}+q_{\mathbf{2}} \text { for } q_{1}=2 s_{n}+2 n \text { and } q_{\mathbf{2}}=s_{n}+2 n .
$$

More precisely, concerning 2, Nash proves that if $f_{0}: X \rightarrow \mathbb{R}^{q}$ is a free $C^{\infty}$ immersion, then

[ $\star$ there exists a $C^{3}$-neighbourhood $G_{0}^{3}$ of the induced metric $g_{0}=\left(D f_{0}\right)^{\star}\left(\sum_{i=1}^{q} d y_{i}^{2}\right)$ in the space of $C^{3}$-metrics on $X$, such that all $C^{r}$-metrics $g \in G_{0}^{3}$ can be induced by $C^{r}$-maps $f: X \rightarrow \mathbb{R}^{q}$.

This, Nash observes, applies to generic $C^{\infty}$-maps $f_{0}: X \rightarrow \mathbb{R}^{q_{2}}$, since these are free for $q_{2} \geq s_{n}+2 n$. (Generic linear projections of a "free" $X$ from some huge $\mathbb{R}^{Q}$ to $\mathbb{R}^{q_{2} \geq s_{n}+2 n}$ are free for the same reason such projections to $\mathbb{R}^{q \geq 2 n}$ are immersions.)

What is most significant here is Nash's proof of [ $\star$ ] that relies on two points of different nature.

[ Infinitesimal invertibility of the metric inducing operator $\mathcal{D}: f \mapsto g$ at free maps.

Indeed, if $f_{0}: X \rightarrow \mathbb{R}^{q}$ is free, then, as we saw in section 3.6.2, the linearisation $L_{f_{0}}: f^{\prime} \mapsto g^{\prime}$ of $\mathcal{D}$ admits a right inverse $M_{f_{0}}: g^{\prime} \mapsto f^{\prime}$ where $M$ is a differential operator in $\left(f_{0}, g^{\prime}\right)$ that is linear of order zero in $f^{\prime}$ and is non-linear of the second order in $f_{0}$.

However easy, this constitutes the essential "geometric" (algebraic?) element of $[\star]$.

[®] Implicit function/perturbation theorem.

This is a general property of non-linear operators. Nash proves it using the notations adapted to the specific problem he solves, but his argument does not use anything particular about isometric immersions. (This feature of Nash's proof was brought to light by J. Schwartz in 1960, who christened this Nash's implicit functional theorem [67.)

But it turned out to be surprisingly difficult to properly reformulate what Nash had actually proved in his 1956 paper in general terms. (This is witnessed by the multiplicity of attempts by many authors at generalising Nash.)

It sounds easy enough in the language of infinitesimal invertibility.

\section{Nash Inverse Function Theorem.}

Let $\mathcal{D}: f \mapsto g$ be a $C^{\infty}$-smooth differential operator of order $r$ between spaces of functions, maps or sections of fibrations over a smooth manifold $X$, such that the linearisation $L_{f}: f^{\prime} \rightarrow g^{\prime}$ of $\mathcal{D}$ is right invertible at all $f$ in a $C^{d}$-neighbourhood of some $C^{\infty}$-smooth $f_{0}$ by a $C^{\infty}$-smooth differential operator $M_{f}\left(g^{\prime}\right)$ that has order $s$ in $g^{\prime}$ and order $d$ in $f_{0}$.

\footnotetext{
${ }^{36}$ In fact, Nash proves the following weak version of $\mathbf{1}$ :

1 ) the set $G_{1}$ of $C^{r}$-metrics that are $C^{r}$-approximately inducible from $\mathbb{R}^{q_{1}=2 s_{n}+2 n}$ contains a $C^{1}$-open subset $G_{1}^{\prime} \subset G_{1}$.

This he compensates with a use of his $C^{1}$-immersion theorem by

2 ) the set $G_{2}$ of metrics that are $C^{r}$-inducible from $\mathbb{R}^{q_{2}=s_{n}+2 n}$ contains a subset $G_{2}^{\prime} \subset G_{2}$ that is $C^{r}$-open and $C^{1}$-dense.

This suffices for decomposition $g=g_{1}^{\prime}+g_{2}^{\prime}$ for all $C^{r}$-metrics $g$ and some $g_{1}^{\prime} \in G_{1}^{\prime}$ and $g_{2}^{\prime} \in G_{2}^{\prime}$ depending on $g$.
} 
Then the operator $\mathcal{D}$ is right invertible by some $\mathcal{D}^{-1}=\mathcal{D}_{f_{0}}^{-1}: g \mapsto f$, i.e.,

$$
\mathcal{D} \circ \mathcal{D}^{-1}(g)=g
$$

where $\mathcal{D}^{-1}$ is defined on some $C^{\sigma+s}$-neighbourhood $\mathcal{V}_{0}^{\sigma+s}$ of $g_{0}=\mathcal{D}\left(f_{0}\right)$ for $\sigma=$ $1+\max (d, 2 r+s)$ and where the regularity class of $g$ goes down by $s$ under $\mathcal{D}^{-1}$,

if $g \in \mathcal{V}=\mathcal{V}_{0}^{\sigma+s}$ is $C^{\sigma+s+\kappa}$-smooth, then $f=\mathcal{D}^{-1}(g)$ is $C^{\sigma+\kappa}$-smooth.

Moreover - this was proved by Nash ten years later in 1966-if $\mathcal{D}, M, f_{0}$, and $g$ are real analytic, then $\mathcal{D}^{-1}(g)$ is also real analytic 37

About Nash's Proof. Think of $\mathcal{D}$ as a smooth fibration

$$
\mathcal{U} \stackrel{\mathcal{D}}{\rightarrow} \mathcal{V} \text { for } \mathcal{U} \ni f_{0}, \quad \mathcal{V} \ni g_{0} \text { and } \mathcal{D}\left(f_{0}\right)=g_{0},
$$

of some neighbourhood $\mathcal{U}$ of $f_{0}$ in a relevant function space over $\mathcal{V}$, and visualise inversions $M_{f}(g)$ of $L_{f}, f \in \mathcal{U}$, as a field of horizontal tangent spaces $H_{f} \subset T_{f}(\mathcal{U})$ that are images of the tangent spaces $T_{g}(V)$ under $M_{f}(g)$.

Given $g_{1} \in \mathcal{V}$, take a path $g_{\theta}$ in $\mathcal{V}$ from $g_{0}=\mathcal{D}\left(f_{0}\right)$ to $g_{1}$ and observe that the final point of the horizontal lift $f_{\theta}$ of this path to $\mathcal{U}$ solves the equation $\mathcal{D}\left(f_{1}\right)=g_{1}$ and thus, furnishes inversion of $\mathcal{D}$.

The construction of $f_{\theta}$ formally amounts to solving ODEs defined by the line field that is the restriction of $H$ to the $\mathcal{D}$ pull-back of the path $g_{\theta}$. But when you try to solve these ODEs, you observe that differentiations in $L$ and in $M$ take you out of any particular (localised Banach) space $C^{k}$ where solution of ODE is possible.

A seemingly absurd idea is to compensate for this loss of regularity by inserting smoothing operators to these ODEs. Of course, the resulting "smoothed lift", say $f_{\theta}^{\circ}$, will deviate from its goal $f_{1}=\mathcal{D}^{-1}\left(g_{1}\right)$, but you keep redirecting such $f_{\theta}^{\circ}$ all the time to make it eventually arrive at $f_{1}$ where $\mathcal{D}\left(f_{1}\right)=g_{1}$.

"Obviously" this cannot work: the law of preservation of regularity seems as unbreakable in analysis as the law of conservation of energy in physics.

But Nash has already broken this "law" in his $C^{1}$-theorem (1954), then he has done it in the $C^{\infty}$-case (1956), and he has repeated this feat in his $C^{a n}$-paper (1966) once again.

Thus, Nash designed an ODE with properly $\theta$-dependent smoothing built into it, we call it the Nash process, and then showed - this is an unpleasant PURELY MECHANICAL COMPUTATION 38 that

if $g_{1}$ is sufficiently $C^{\sigma+s}$-close to $g_{0}=\mathcal{D}\left(f_{0}\right)$, then Nash's process converges to the desired $f_{1}$ for which $\mathcal{D}\left(f_{1}\right)=g_{1}$.

(The detailed proof of all this takes two to three pages in the notation of section 2.3 in 35. .)

Albeit certain properties of $\mathcal{D}^{-1}$, especially its continuity in $f_{0}$ and locality 39 are essential for microflexibility, and the $h$-principle (see 2.3 in [35]) and the operator $M=M_{f}\left(g^{\prime}\right)$, in general, need not be a differential operator, since Nash's construction of $\mathcal{D}^{-1}$ applies in a wider range of situations.

\footnotetext{
${ }^{37}$ The operator $\mathcal{D}^{-1}$ is by no means unique. The one constructed by Nash in his 1956 63] paper was not respectful of real analyticity; this was amended by Nash in 1966 64].

${ }^{38}$ Part of the "mechanics" consists in evaluation of exponents of products of derivatives coming from the chain rule and Leibniz's formula in the course of differentiating non-linear operators applied to functions. Possibly, a suitable algebraic formalism in the spirit of tropical geometry may replace combinatorial fiddling involved in such evaluation.

${ }^{39}$ Nash's process, as it stands, is non-local on the underlying manifold $X$, but its discretised (in $\theta$ ) version suggested by Hörmander (1976) (see 47] and 2.3 in [35]) is (quasi-)local.
} 
For instance Nash's proof automatically extends to all $M$ that satisfy the same kind of estimates as differential operators do. However, just enlisting these estimates takes as much space and effort as proving Nash's theorem.

Also, Nash's proof is not bound to $C^{r}$-spaces of functions and it can be executed in terms of abstract graded Fréchet spaces. This was implemented by R. Hamilton (1982, 43]), but the background needed just to formulate such an abstract implicit function theorem spreads over 100 pages.

In truth, "spaces" play only notational roles in Nash's proof that consists in "processing numerical estimates", and these do not have to be linked to any "space" at all.

The fixed point property of this process established by Nash (this is where PURELY MECHANICAL COMPUTATION comes in) was reformulated for abstract Nash processes in [34, 40 That was meant to imply all conceivable versions of Nash's theorem; likely it does, but it is too heavy to be usable in practice 41

Probabaly, the eventual general theorem, in order to be painlessly applicable to concrete problems, should be even more "abstract" and go significantly beyond the scope of Nash's construction, including, in particular, the variation suggested by Günther.

Analytic Perturbations. Since the inversion $M$ of the linearised operator $L_{\mathcal{D}}$ is a differential operator algebraically depending on $L_{\mathcal{D}}$, what this $M=M_{f}(g)$ actually does is a representation of the perturbation equation

$$
\mathcal{D}\left(f_{0}+f_{\varepsilon}^{\prime}\right)=\mathcal{D}\left(f_{0}\right)+g_{\varepsilon}^{\prime}
$$

in the evolution form of first order with respect to $\varepsilon$. But since $M_{f}(g)$ may (it usually does) have order $>1$ in $f$, it is not Cauchy-Kovalevskaya.

Sometimes (always?) differentiating such an equation and excluding undesirable higher order derivatives, one may bring it to Cauchy-Kovalevskaya form and then

the solvability of this equation in the analytic case for $g_{\varepsilon}^{\prime}=\varepsilon g^{\prime}$ with real analytic $g^{\prime}$ follows from the Cauchy-Kovalevskaya theorem 42

For example, Gauss's formula-Theorema egregium - furnishes such CauchyKovalevskaya reduction for the isometric imbedding equations and

the Cauchy-Kovalevskaya theorem + Nash's approximate immersion theorem (see section 3.8) imply the isometric imbedding theorem for compact $C^{a n}$-manifolds.

But, in fact, a fragment of the Cauchy-Kovalevskaya proof (e.g., in the form of Newton iterations) applies to the perturbation equation in the $C^{a n}$-case in its original (non-Cauchy-Kovalevskaya) form and delivers solution $f^{\prime}$ of the equation $\mathcal{D}\left(f_{0}+f_{\varepsilon}^{\prime}\right)=\mathcal{D}\left(f_{0}\right)+\varepsilon g^{\prime}$ for small $\varepsilon>0$. (This was pointed out in [33] and in [39].)

Approximation and Regularity. The continuity of the operator $\mathcal{D}^{-1}$ implied by Nash's estimates allows regularisation solutions of the equation $\mathcal{D}(f)=g$ by

\footnotetext{
${ }^{40}$ This was needed in 34 for construction of local isometric $C^{\infty}$-immersions $X^{n} \rightarrow \mathbb{R}^{s_{n}-1}$, where the obviouos inversion of $L_{\mathcal{D}}$ comes by solution of ODE that is not a differential operator $M$.

${ }^{41}$ Also "locality", which was used throughout the paper 34, was not properly incorporated there to the abstract Nash process. The necessary (quite simple) adjustment of Nash's process needed for the sake of microflexibility and the h-principle, similar to what Hörmander does in his paper, is provided in 2.3 of 35 .

${ }^{42}$ Nash's analytic perturbation theorem is significantly stronger: it delivers $C^{a n}$-solutions of the perturbation equation for $g_{\varepsilon}^{\prime}$ that are $\varepsilon$-small in $C^{k}$-norm for some finite $k$, namely $k=$ $1+\max (d, 2 r+s)+s$.
} 
approximation (see 2.3.2 in [35]). For instance, if $g$ is a Riemannian $C^{\infty}$-metric on a manifold $X$, then

free isometric $C^{k}$-immersions $f:(X, g) \rightarrow \mathbb{R}^{q}$ can be finely $C^{l}$-approximated by $C^{\infty}$-smooth isometric immersions, provided

$$
k>3 \text { and } l<k
$$

and the same remains true with the $C^{a n}$ in place of $C^{\infty}$.

Question. Can free isometric $C^{3}$-immersions of $C^{\infty}$-manifolds be $C^{2}$-approximated by isometric $C^{\infty}$-immersions?

Local Immersions by Perturbation + Scaling. Perturbations that are local in the spaces of maps can be, albeit rarely, globalised in the presence of non-compact groups of symmetries.

For instance, perturbation and scaling of free isotropic immersions $X \rightarrow \mathbb{R}^{q_{+}, q_{-}}$ yields isometric immersion of all compact $(X, g)$ to this $\mathbb{R}^{q_{+}, q_{-}}$(see section 3.6.1).

Now, let us use scaling in $\mathbb{R}^{q}$ to obtain local free isometric $C^{\infty}$-immersions of $C^{\infty}$-manifolds to $\mathbb{R}^{q}$ for $q=s_{n}+n=\frac{n(n+1)}{2}+n$.

(Originally, this was proven by Robert Greene by applying Nash's perturbation to approximte solutions of Janet's equations.)

Start by observing that the unit ball $B^{n} \subset \mathbb{R}^{n}$ with its induced flat Euclidean metric $g_{0}=\sum_{i=1}^{n} d x_{i}^{2}$ admits

$$
\text { a free isometric } C^{a n} \text {-embedding } f_{0}:\left(B^{n}, g_{0}\right) \rightarrow \mathbb{R}^{s_{n}+n} \text {. }
$$

Proof. Generically isometrically bend $\mathbb{R}^{n}$ in $\mathbb{R}^{n+1} \supset \mathbb{R}^{n}$, then similarly generically bend $\mathbb{R}^{n+1}$ in $\mathbb{R}^{n+2}$, etc., with the final bending being that of $\mathbb{R}^{s_{n}+n-1}$ in $\mathbb{R}^{s_{n}+n}$.

It is easy to see that "genericity" guarantees freedom of the resulting composed map $\mathbb{R}^{n} \rightarrow \mathbb{R}^{s_{n}+n}$ in a small ball around $\mathbf{0} \in \mathbb{R}^{n}$ that can be assumed to be of unit size 43

Next, given an arbitrary $C^{r}$-metric $g$ on $X$ in a neighbourhood $U_{0} \subset X$ of a point, $x_{0} \in X$, take local coordinates that identify this $U_{0}$ with a neighbourhood of $\mathbf{0}$ in $\mathbb{R}^{n}$ such that $g$ becomes $g=g_{0}+g^{\prime}$, where $g_{0}$ is the Euclidean metric and where $g^{\prime}$ is a $C^{r}$-smooth quadratic form that vanishes at $\mathbf{0}$.

The $\delta^{-1}$-scaling map $x \mapsto \delta^{-1} x$ from the $\delta$-ball $B^{n}(\delta) \in \mathbb{R}^{n}$ around $\mathbf{0} \in \mathbb{R}^{n}$ to the unit ball $B^{n} \subset \mathbb{R}^{n}$ sends $g^{\prime}$ to the form $g_{\delta}^{\prime}$ on $B^{n} \subset \mathbb{R}^{n}$, where obviously, $g_{\delta}^{\prime}$ converges to zero on $B^{n}$ in the $C^{r}$-topology,

$$
\left\|g_{\delta}^{\prime}\right\|_{C^{r}} \rightarrow 0 \text { for } \delta \rightarrow 0 .
$$

It follows that

if $r>2$, then by Nash's theorem, $f_{0}$ can be perturbed to $f$ which induces $g_{0}+g_{\delta}^{\prime}$ on $B^{n}$ by a $C^{r}$-map $f^{\prime}: B^{n} \rightarrow \mathbb{R}^{s_{n}+n}$, and by composing this $f^{\prime}$ with the map $y \mapsto \delta y, y \in \mathbb{R}^{s_{n}+n}$, we obtain the required free isometric map $B^{n}(\delta, g) \rightarrow \mathbb{R}^{s_{n}+n}$.

Immersions to non-Euclidean manifolds $Y^{q}=\left(Y^{q}, \tilde{g}\right)$. If we scale $Y^{q}$ by $\left(Y^{q}, \tilde{g}\right) \sim$ $\left(Y^{q}, \lambda \tilde{g}\right)$, then, at each point $y_{0} \in Y^{q}$, the geometry of $Y^{q}$ converges to that of $\mathbb{R}^{q}=T_{y_{0}}\left(Y^{q}\right)$ for $\lambda \rightarrow \infty$.

\footnotetext{
${ }^{43}$ Actually, it is not hard to arrange such a bending that would terminate with a free isometric map from all of $\mathbb{R}^{n}$ to $\mathbb{R}^{s_{n}+n}$.
} 
Therefore, perturbation+scaling work here as well, and, more generally, this argument shows that

an arbitrary (possibly singular) $C^{r}$-form $g$ on $X$, where $r>2$, of type $\left(n_{+}, n_{-}, n_{0}\right)$, $n_{+}+n_{-}+n_{0}=n=\operatorname{dim}(X)$, at a point $x_{0}$ admits a local free isometric $C^{r}$-imbedding to a given pseudo-Riemannian $C^{a n}$-manifold $Y=(Y, \tilde{g})$, provided

$$
q_{ \pm} \geq n_{ \pm}+n_{0} q=\operatorname{dim}(Y)=q_{+}=q_{-} \geq s_{n}+n+n_{0} .
$$

\section{QUESTIONS}

What is the actual regularity class of $\tilde{g}$ needed for the validity of the Nash perturbation theorem for these embeddings?

57 How well does the bending+scaling argument generalise to local "isometric immersions" of forms of degrees $p \geq 3$ ?

Is there any chance for globalisation of bending+scaling?

Elliptic Immersions of Surfaces with Codimensions $\geq 1$. Call an immersion of a surface to a Riemannian manifold $f_{0}: X \rightarrow Y=Y(\tilde{g})$ elliptic if it admits a normal vector field on which the second fundamental form is positive, or, equivalently, if $f_{0}$ extends to $X \times[-\varepsilon, \varepsilon] \rightarrow Y$ such that $X=X \times 0 \subset X \times[-\varepsilon, \varepsilon]$ is locally convex in $X$ with respect to the metric in $X \times[-\varepsilon, \varepsilon]$ induced from $Y$.

For example, all surfaces in the 3 -sphere $S^{3} \subset \mathbb{R}^{4}$ are elliptic in $\mathbb{R}^{4}$.

If $X=S^{2}$ or if $X$ is compact connected with non-empty boundary, then Herman's Weyl's perturbation theorem says that the metric inducing operator $f \mapsto g=$ $\left(D f_{0}\right)^{\star}(\tilde{g})$ is open at $f_{0}$, say, for $C^{\infty}$-smooth $f_{0}$ and $\tilde{g}$ :

the metrics $g$ that are $C^{\infty}$-close to the induced metric $g_{0}=\left(D f_{0}\right)^{\star}(\tilde{g})$ are inducible by $C^{\infty}$-maps $f: X \rightarrow Y$.

And it seems not hard to show that this remains true for all compact $X$ if $\operatorname{dim}(Y) \geq 4$.

Question. Do all compact $C^{\infty}$-surfaces $X=(X, g)$ admit isometric elliptic $C^{\infty}$ _ immersions to $\mathbb{R}^{4}$ ?

\subsection{Nash Decomposition, Conformal Twist, and $C^{\infty}$-Approximate Em- beddings.}

Let $f_{0}: X \rightarrow\left(\mathbb{R}^{q}, \sum_{j} d y_{j}^{2}\right)$ be a smooth map with constant norm, $\left\langle f_{0}, f_{0}\right\rangle_{\mathbb{R}^{q}} \varepsilon^{2}>0$, let $g_{0}$ be the induced quadratic form on $X$ written as $g_{0}=\left\langle d f_{0}, d f_{0}\right\rangle$, and let $\psi(x)$ be a $C^{1}$-function on $X$. Then the quadratic form $g_{\varepsilon}$ on $X$ induced by the product $f_{\varepsilon}=\psi f_{0}: X \rightarrow \mathbb{R}^{q}$ satisfies

$$
g_{\varepsilon}=\psi^{2} g_{0}+\varepsilon^{2}(d \psi)^{2} \text {. }
$$

Indeed, since

$$
\left\langle f_{0}, f_{0}\right\rangle=\operatorname{const}(=\varepsilon) \Rightarrow\left\langle d f_{0}, f_{0}\right\rangle=0
$$

the identity

$$
\left\langle d\left(\psi f_{0}\right), d\left(\phi f_{0}\right)\right\rangle=\psi^{2}\left\langle d f_{0}, d f_{0}\right\rangle+\varepsilon^{2}(d \psi)^{2}
$$

follows by Leibniz's formula.

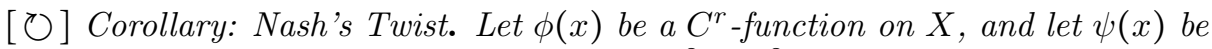
$C^{1}$. Then, given $\varepsilon>0$, the quadratic form $g=\psi^{2}(d \phi)^{2}$ can be represented as

$$
g=\left(d \varphi_{\varepsilon}\right)^{2}+\left(d \chi_{\varepsilon}\right)^{2}-\varepsilon^{2}(d \psi)^{2},
$$

where $\varphi_{\varepsilon}$ and $\chi_{\varepsilon}$ are $C^{r}$-functions on $X$. 
Proof. Compose $f: X \rightarrow \mathbb{R}$ with the isometric immersion of the line $\mathbb{R}$ onto the $\varepsilon$-circle in the plane, and apply the above to the resulting map $f_{0}=e_{\varepsilon} \circ f: \mathbb{R} \rightarrow$ $S^{1}(\varepsilon) \subset \mathbb{R}^{q=2}$.

$\left[\square^{+}\right] \sum \psi_{\nu} d \phi_{\nu}^{2}$-Decomposition Revisited (See [ $\left.\square^{\prime}\right]$ on p. 202). All $C^{r}$-metrics $g$ on $n$-dimensional manifolds $X$ admit decompositions

$$
g=\sum_{\nu=1}^{q_{1}} \psi_{\nu}^{2} d \phi_{\nu}^{2}, q_{1}=s_{n}+n, s_{n}=\frac{n(n+1)}{2},
$$

where the functions $\phi_{\nu}=\phi_{\nu}(x)$ are real analytic and $\psi_{\nu}=\psi_{\nu}(x)$ are $C^{r} 44$

This is shown by an obvious reduction to the following $h$-principle that can be proved by convex integration 45

Let an $n$-manifold $X$ admit $q_{1}$ linear differential forms $l_{\nu}$, such that $l_{\nu}^{2}(x)$ linearly span the fibres $\mathcal{G}_{x}(X)$ of the symmetric square $\mathcal{G}(X)$ of the cotangent bundle (that has rank $=s_{n}$ ) at all points $x \in X$, and let $g$ be a continuous Riemannian metric on $X$ that is a section of $\mathcal{G}(X)$ thought of as a family $g(x) \in \mathcal{G}_{x}(X)$.

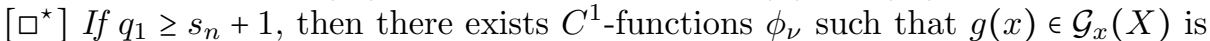
contained in the interior of the convex hull of the set of vectors $\left\{\left(d \phi_{\nu}(x)\right)^{2}\right\} \subset \mathcal{G}_{x}(X)$ for all $x \in X$.

With this we conclude to the following

$\left[\circlearrowright+\square^{+}\right]$Nash's $C^{r}$-Approximate Immersion Theorem. Let $g$ be a $C^{r}$-metric on a $C^{a n}$-manifold $X$. Then, for all $\varepsilon>0$, there exist $C^{r}$-functions $\phi_{\nu}$ on $X$, $\nu=1, \ldots, q_{1}=s_{n}+n$, and $C^{a n}$-immersions $f_{\varepsilon}: X \rightarrow \mathbb{R}^{2 q_{1}}$ such that the metrics induced by these immersions satisfy

$$
\left(D f_{\varepsilon}\right)^{\star}\left(\sum_{j=1}^{2 q_{1}} d y_{j}^{2}\right)=g+\varepsilon^{2} \sum_{\nu=1}^{q_{1}} d \phi_{\nu}^{2} .
$$

Questions

What is the minimal number $q_{\diamond}$, such that all $C^{\infty}$-smooth Riemannian metrics $g$ on $X$ admit decompositions $g=\sum_{\nu=1}^{q_{\diamond}} \psi_{\nu}^{2} d \phi_{\nu}^{2}$ with $C^{\infty}$-smooth $\phi_{\nu}$ and $\psi_{\nu}$ ?

The above stated $h$-principle [ $\square^{\star}$ ] allows $q_{\diamond}=s_{n}+1$ for stably parallelisable manifolds $X$, e.g., homeomorphic to $S^{n}$, and this seems not hard to prove for all $X$ with a special treatment of the locus of those $x \in X$ where the vectors $\left(d \phi_{\nu}(x)\right)^{2} \in \mathcal{G}_{x}$ fail to span all of $\mathcal{G}_{x}$.

But what we truly want is such a decomposition with $2 q_{\diamond} \approx s_{n}$.

More generally:

What are $q_{\diamond}$ and $q_{\circ}$ such that all $C^{\infty}$-smooth $g$ admit $C^{\infty}$-decompositions

$$
g=\sum_{\nu=1}^{q_{\diamond}} \psi_{\nu} d \phi_{\nu}^{2}+\sum_{j=1}^{q_{\diamond}} d f_{j}^{2} ?
$$

Does the inequality $2 q_{\diamond}+q_{\diamond} \geq s_{n}$, or at least $2 q_{\diamond}+q_{\diamond}=s_{n}+O(n)$, suffice for this?

Notice in this regard that if $n=2$, then

- geodesic coordinates provide such local decompositions with $q_{\diamond}, q_{\circ}=1$;

- decompositions with $q_{\diamond}=1$ and $q_{\circ}=0$ are possible for all open surfaces

\footnotetext{
${ }^{44}$ Strictly positive $\psi$ and $\psi^{2}$ are interchangeable for our purposes.

${ }^{45}$ This is easy, and if $n \geq 3$, it may remain valid for $q_{1}=s_{n}$, but I have not honestly checked anything.
} 
by elementary conformal geometry;

- closed surfaces, obviously, need $q_{\diamond}+q_{\diamond} \geq 3$;

- the question is open for $q_{\diamond}=1$ and $q_{\circ}=2$.

\subsection{Nash Twist in non-Riemannian Categories.}

Nash decomposition makes sense for forms $g$ of all degree $p \geq 2$

$$
g=\sum_{\nu} \psi_{\nu} d \phi_{\nu}^{p}
$$

where we insist on $\phi_{\nu}>0$ for positive forms $g$.

Everything said in the previous section - results as well as questions - extend to $C^{r}$-smooth $\sum_{\nu} \psi_{\nu} d \phi_{\nu}^{p}$-decompositions for all $p$, where, however, details need be to worked out.

And much of this extends to convex cones of more general tensorial-like structures (see section 4.2).

But one cannot freely replace $\psi$ by $\psi^{p}$ in such a decomposition if one works with polynomial forms and maps.

For instance, the existence of a polynomial Nash twist depending on maps $e$ from $\mathbb{R}$ to the "unit sphere" in $\mathbb{R}^{q_{+}, q_{-}}$- this sphere contains straight lines for $q_{ \pm} \geq 1$ and $q_{+}+q_{-} \geq 3$ - does not directly lead to polynomial maps that would induce all forms $g$ from pseudo-Euclidean spaces.

63 Question. Which symmetric differential forms $g$ of degree $p \geq 3$ on Euclidean spaces are decomposable as $\sum_{\nu} \psi_{\nu}^{p} d \phi_{\nu}^{p}$ with polynomial or with entire holomorphic functions $\psi_{\nu}$ and $\phi_{\nu}$ ?

If $p \geq 3$, then "twisted" maps $e: \mathbb{R} \rightarrow\left(\mathbb{R}^{m}, h\right)$ that nicely behave under multiplications by scalar functions, i.e., such that

$$
(D(\psi \circ e))^{\star}(h)=\psi^{p}(D \psi)^{\star}(h)+(d \psi)^{p},
$$

must satisfy, besides the relations

$$
h(e)=\varepsilon \text { and }(D e)^{\star}(h)=d x^{p},
$$

$p-2$ additional differential equations.

For instance, if $p=3$, the extra equation can be written as

$$
\left\langle e, e, \frac{d e}{d x}\right\rangle_{h}=0
$$

where $\langle., ., .\rangle_{h}$ denotes the 3 -linear form associated with $h$, which, in the formula $h(e)=\varepsilon$, was regarded as a homogeneous polynomial on $\mathbb{R}^{m}$.

It follows that

differential forms $g$ on $\mathbb{R}^{n}$ of an arbitrary degree $p=2,3, \ldots$ with polynomial coefficients can be induced by maps to $\left(\mathbb{R}^{q}, \sum_{j} d y_{j}^{p}\right)$ that are given by algebraic (but not polynomial) expressions in solutions of a single (universal) ordinary differential equation.

Question. Are the complexifications of these differential equations, e.g.,

$$
h(e)=1,(D e)^{\star}(h)=d x^{3} \text {, and }\left\langle e, e, \frac{d e}{d x}\right\rangle_{h}=0 \text { for } p=3,
$$

solvable by entire holomorphic maps $e: \mathbb{C} \rightarrow \mathbb{C}^{m}$ ? 


\subsection{Microflexibility, Flexibility, and the $h$-Principle.}

We are mainly concerned here with the sheaf $\Phi$ of free isometric $C^{\infty}$-immersions between pseudo-Riemannian, e.g., Riemannian, $C^{\infty}$-manifolds $X=(X, g) \rightarrow Y=$ $(Y, \tilde{g})$. Thus $\Phi(U)$, for open $U \subset X$, stands for the space of such immersions from $U$ to $Y$.

Flexibility. Recall that an abstract topological, better to say continuous, sheaf $\Phi$ on $X$ is an assignment of topological spaces $\Phi(U)$ to all open subsets $U \subset X$ and of continuous maps $\Phi(I): \Phi\left(U^{\sim}\right) \rightarrow \Phi(U)$ to all inclusions $U \stackrel{I}{\subset} U^{\sim}$ between open subsets in $X$.

If $C \subset X$ is a compact subset, then $\Phi(C)$ denotes the space of $C$-germs of sections of $\Phi$ over arbitrarily small open subsets $U \supset C$. More precisely, $\Phi(C)$ is the inductive limit of the spaces $\Phi(U)$ over all neighbourhoods $U \supset C$.

One calls $\phi \in \Phi(U)$ sections of $\Phi$ over $U$, and $\Phi(I)(\phi)$ for $U \stackrel{I}{\subset} U^{\sim}$ are called restrictions of $\phi$ from $U^{\sim}$ to $U$.

A sheaf $\Phi$ is called flexible if the restriction maps $\Phi(I): \Phi\left(C^{\sim}\right) \rightarrow \Phi(C)$ are Serre fibrations for all pairs of compact subsets $C \stackrel{I}{\subset} C^{\sim} \subset X$, i.e., if continuous paths $\phi_{t}$ in $\Phi(C)$ can be (non-uniquely) lifted to paths in $\Phi\left(C^{\sim}\right)$ starting from a given point $\tilde{\phi} \in \Phi\left(C^{\sim}\right)$ over $\phi_{0} \in \Phi(C)$. Moreover, by the definition of fibration, such lifts must exist for continuous families of paths $\phi_{t, p}$ parametrised by finite polyhedra $P \ni p$.

Flexibility imitates the homotopy extension property (Borsuk Lemma) for the sheaves of all smooth (or just continuous) maps.

Warning. Homotopies of sections over open subsets $U$ that are not supported strictly inside $U$ usually do not extend outside $U$. This is why one needs to operate with sections over neighbourhoods $U \supset C$ of compact subsets $C \subset X$ where the actual domains of definitions $U \supset C$ of homotopies over $C$ may shrink, yet remaining $\supset C$, in the course of their extension to $U^{\sim} \subset C^{\sim} \supset C$.

Microflexibility. A sheaf $\Phi$ is called microflexible if the initial phases of paths $\phi_{t}, 0 \leq t \leq 1$, and of families of these are liftable, where "initial" means for $0 \leq t \leq \varepsilon$ where $\varepsilon>0$ may depend on the path.

For instance, the sheafs $\Phi_{\text {Imm }}$ of smooth immersions $X \rightarrow Y$ and $\Phi_{\text {free }}$ of (nonisometric!) free immersions $X \rightarrow Y$ are, obviously, microflexible.

But $\Phi_{\text {imm }}$ is flexible only if $n=\operatorname{dim}(Y)>q=\operatorname{dim}(X)$, and this property of immersion, proven by Smale, is by no means at all obvious - it is, in fact, the key geometric ingredient of the Smale-Hirsch immersion theory.

Also, one knows (see 2.2 in [35] and references therein) that the sheaf $\Phi_{\text {free }}$ is flexible for $q>s_{n}+n, s_{n}=\frac{n(n+1)}{2}$, but

flexibility of $\Phi_{\text {free }}$ for $q=s_{n}+n$ and $n \geq 2$ is a long standing unsolved difficult problem.

Flexibility is a strong property: according to Smale and Hirsch,

\section{flexibility implies the h-principle for $\Phi$.}

Abstractly speaking, this means that every continuous in $x \in X$ family $\psi_{x} \in$ $\Phi\left(U_{x}\right)$ of sections of $\Phi$ over (arbitrarily small) neighbourhoods $U_{x} \ni x$ in $X$ can be deformed to an "integrable family" $\phi_{x}$, where all these $\phi_{x}$ come by restrictions from a single $\phi \in \Phi(X) 46$

\footnotetext{
${ }^{46}$ Flexibility is no good for proving the $h$-principle in the context of real analytic maps and even less so of complex analytic maps, since these are not microflexible. But a refined concept
} 
Microflexibilty, unlike flexibility, is cheap as far as differential topology is concerned, but when it comes to sheaves of solutions of differential equations, microflexibility may look, at least in the eye of an analyst, as improbable as flexibility.

Remarkably, Nash's theorem delivers

microflexibility for the sheaves $\Phi=\Phi_{\mathcal{D}, g}$ of $C^{\infty}$-solutions $f$ of differential

equations $\mathcal{D}(f)=g$ for infinitesimally invertible differential operators $\mathcal{D}$

on smooth manifolds $X$.

Then, under favourable circumstances, e.g., in the presence of strong symmetries of $\Phi$, one can prove flexibility and, hence, the $h$-principle for $\Phi$ that reduces the solvability of the equation $\mathcal{D}(f)=g$ to the existence of a continuous section of some auxiliary (possibly singular) fibration over $X$.

Below is an instance of where a combination of ideas of Nash and Smale directly leads to a geometric result similar to Hirsch immersion theorem for open manifolds.

Let $p: X \rightarrow X_{0}$ be a $C^{\infty}$-smooth submersion (i.e., the differential $D p(x)$ : $T_{x}(X) \rightarrow T_{p(x)}\left(X_{0}\right)$ is surjective for all $\left.x \in X\right)$, and let the pull-backs $p^{-1}\left(x_{0}\right) \subset X$ be open (i.e., no compact components) submanifolds (necessarily) of dimension $m=\operatorname{dim}(X)-\operatorname{dim}\left(X_{0}\right)$ for all $x_{0}$ in the image $p(X) \subset X_{0}$.

(Instructive examples are where $X$ is an open manifold mapped to a single point $X_{0}=\left\{x_{0}\right\}$ or $X=X_{0} \times \mathbb{R}$ projected to $X_{0}$.)

Let $g$ be the quadratic differential form on $X$ that is induced by $p: X \rightarrow X_{0}$ from some $C^{\infty}$-smooth form $g_{0}$ on $X_{0}$, and let $Y=(Y, \tilde{g})$ be a $C^{\infty}$ smooth pseudoRiemannian manifold.

Then free isometric $C^{\infty}$-immersions $f:(X, g) \rightarrow(Y, \tilde{g})$ satisfy the $h$-principle.

A Word about the Proof. Flexibility is derived here from microflexibility with the use of the (quite large!) group of diffeomorphisms of $X$ that preserve the fibres $p^{-1}\left(x_{0}\right)$ of the map $p: X \rightarrow X_{0}$, where this is the key-the action of this group on $X$ preserves $g$, and hence, our sheaf $\Phi$ of free isometric immersions $(X, g) \rightarrow Y$.

Corollary. Let the form $g_{0}$ on $X_{0}$ be non-singular of type $\left(n_{+}, n_{-}\right), n_{+}+n_{-}=n_{0}=$ $\operatorname{dim}\left(X_{0}\right)$, and let the manifolds $(X, g)$ and $(Y, \tilde{g})$ be globally diagonalisable.

(As far as $(Y, \tilde{g})$ is concerned, this means that $Y$ admits a frame of $q=\operatorname{dim}(Y)$ mutually $\tilde{g}$-orthogonal vector fields with $\tilde{g}$-norms \pm 1 , namely $q_{+}$of them with norms +1 and $q_{-}$with norms -1. For example, open subsets $Y$ in the pseudo-Euclidean space $\mathbb{R}^{q_{+}, q_{-}}$are globally diagonalisable. And for $(X, g)$, such a diagonalisation is a field of $n=\operatorname{dim}(X)$ linearly independent mutually $g$-orthogonal vector fields, where, $n_{0}=\operatorname{dim}\left(X_{0}\right)$ of them have $g$-norms \pm 1 and the remaining $m=n-n_{0}$ have $g$ norms zero. For instance, since our form $g$ on $X$ has constant $\operatorname{rank}=\operatorname{dim}\left(X_{0}\right)$, it is diagonalisable if $X$ is contractible.)

Then $(X, g)$ admits a free $C^{\infty}$-smooth isometric immersion to $(Y, \tilde{g})$ in each homotopy class of maps $X \rightarrow Y$ if and only if the type $\left(q_{+}, q_{-}\right)$of the form $\tilde{g}$ on $Y$ satisfies

$$
q_{ \pm} \geq n_{ \pm}+m, m=\operatorname{dim}(X)-\operatorname{dim}\left(X_{0}\right), \text { and } q_{+}+q_{-} \geq s_{n}+n+m, s_{n}=\frac{n(n+1)}{2} .
$$

The above $h$-principle and the corollary remain valid for $C^{a n}$-immersions $X \rightarrow Y$, if the forms $g_{0}$ on $X_{0}$ and $\tilde{g}$ on $Y$, and the map $P: X \rightarrow X_{0}$ are $C^{a n}$.

that generalises flexibility and that is expressed in terms of Cartan pairs works equally well in the analytic and in the smooth categories; see [36, 30] and references therein. 
Moreover, the following seems also within reach for $m>0$. (The case $m=0$ needs new ideas.)

Conjecture. If $X$ is contractible, the form $g_{0}$ is non-singular, and

$$
q_{ \pm} \geq n_{ \pm}+m, \text { and } q_{+}+q_{-} \geq s_{n}+m
$$

then $X$ admits a (non-free!) isometric $C^{\text {an }}$-immersion to $Y$.

(See 3.3.5 in [35] for some results in this direction.)

\subsection{Local Janet's Equations and Semilocal Extension of Isometric Im- mersions.}

Semilocal Extension Problem. Let $(X, g)$ be a smooth $n$-manifold with a quadratic differential form on it, let $X_{0} \subset X$ be a closed subset, and let $g_{0}$ denote the restriction of $g$ to the tangent bundle of $X$ over $X_{0}$, denoted $T(X)_{\mid X_{0}}$.

Let $Y=(Y, \tilde{g})$ be a pseudo-Riemannian manifold that, for simplicity's sake, is assumed $C^{a n}$. Let $f_{0}: X \rightarrow Y$ be a $C^{r_{0}}$-map which is isometric over $X_{0}$ and such that the differential of $D f_{0}: T(X) \rightarrow T(Y)$ restricted to $T(X)_{\mid X_{0}} \subset T(X)$ induces $g_{0}$ from $\tilde{g}$.

("Semilocal" stands for global along $X_{0}$ while local normally to $X_{0}$.)

When does there exist an isometric $C^{r}$-map $f$ from some neighbourhood $U \supset X_{0}$ to $Y$, i.e., $(D f)^{\star}(\tilde{g})_{\mid U}=g_{\mid U}$, such that the restriction of $f$ to $X_{0}$ equals that of $f_{0}$,

$$
f_{\mid X_{0}}=\left(f_{0}\right)_{\mid X_{0}} ?
$$

If $X_{0}$ is a $C^{a n}$-hypersurface and $U \subset X$ is a split neighbourhood of $X_{0} \subset X$, $U=X \times[\varepsilon, \varepsilon]$, then Janet (1926) showed in the Riemannian case (see below) that the expression of the curvature of $g$ in terms of the (extrinsic) curvature $U \stackrel{f}{\subset} Y$ (Gauss's Theorema egregium, 1827) brings the first order system of isometric immersion equations $\left\langle\partial_{i} f, \partial_{j} f\right\rangle_{\tilde{g}}=g_{i j}$ for $f: U \rightarrow Y$ to a second order implicit evolution system with the initial conditions defined via the Euler formula (1760) for the squared extrinsic curvature of $f: X_{0} \rightarrow Y$ that is the Pythagorean sum (500 B.C.E.) of the squared curvature of $X_{0} \subset X$ and the squared normal curvature of $X_{0} \subset U \stackrel{f}{\subset} X_{0}$. (This normal curvature depends only on the second fundamental form of $U \stackrel{f}{\subset} X_{0}$ on $T\left(X_{0}\right) \subset T(U)$ by Meusnier's theorem of 1776-1785.)

And the Gaussian expression can be turned to an explicit evolution system amenable to the Cauchy-Kovalevskaya theorem by resolving the Gaussian relation in $\partial_{t t}(f), t \in[-\varepsilon, \varepsilon]$, provided - this was clarified by Burstin (1931) - the map $f_{0}: X_{0} \rightarrow Y$ is free.

In fact, such a resolution is possible, since (the relevant part of the left-hand side of) the Gauss formula is linear in $\partial_{t t}$, where it is composed of $\tilde{g}$-scalar products of $\partial_{t t}(f)$ with the vectors of the first and the second derivatives of $f$, except $\partial_{t t}$ itself.

On the other hand, the Pythagorean Euler-Meusnier relation for the curvatures

$$
K_{f_{0}}^{2}\left(X_{0} \stackrel{f_{0}}{\subset} Y\right)=K_{I}^{2}\left(X_{0} \stackrel{I}{\complement} X\right)+K_{f}^{2}(X \stackrel{f}{\subset} Y)
$$


is solvable in $K_{f}$ on $X_{0}$ if the difference, $K_{f_{0}}^{2}\left(X_{0} \stackrel{f_{0}}{\subset} Y\right)-K_{I}^{2}\left(X_{0} \stackrel{I}{\subset} X\right)$ regarded as a symmetric form of degree four, is positive definite 47

Thus, Burstin shows that if $X$ is a Riemannian $C^{a n}$-manifold and $X_{0} \subset X$ is a $C^{a n}$-hypersurface that is geodesic at some point $x_{0} \in X_{0}$, i.e., the (relative or extrinsic) curvature $K_{I}^{2}\left(X_{0} \stackrel{I}{\subset} X\right)$ vanishes at $x_{0}$, then

[ every free isometric $C^{a n}$-immersion $f_{0}: X_{0} \rightarrow Y$ restricted to a (small) neighbourhood $U_{0} \subset X_{0}$ of $x_{0}$ in $X_{0}$, extends to an isometric $C^{a n}$-immersion $f: U \rightarrow Y$, where $U \subset X$ is a (small) neighbourhood of $x_{0}$ in $X$.

Next, a closer look at the (resolved) Gauss expression shows that

[\%s] if $q=\operatorname{dim}(Y) \geq s_{n}+n, s_{n}=\frac{n(n+1)}{2}$, then this (isometric real analytic) map $f: U \rightarrow Y$ can be chosen free.

Then an obvious induction in $\operatorname{dim}(X)$ shows (Janet-Burstin) that all Riemannian $C^{a n}$-manifolds are locally $C^{n}$-embeddable to $\mathbb{R}^{s_{n}}$.

By the same token, pseudo-Riemannian $C^{a n}$-manifolds $(X, g)$ of type $\left(n_{+}, n_{-}\right)$, $n_{+}+n_{-}=n=\operatorname{dim}(X)$ are locally $C^{\text {an }}$-embeddable to $(Y, \tilde{g})$ of type $\left(q_{+}, q_{-}\right)$, if

$$
q_{ \pm} \geq n_{ \pm} \text {and } q_{+}+q_{-}=q=\operatorname{dim}(Y) \geq s_{n}
$$

see 29,48

QUESTION. What would be a Diophantine version of local isometric immersions?

\section{INFINITESIMAL INVERTIBILITY OF $(D f)^{\star}$, JANET's EqUATIONS, and Hermann Weyl's Tube Formula}

Let us derive an infinitesimal inversion $M=M_{f_{0}}\left(g^{\prime}\right)$ of the metric inducing operator $f \mapsto g=(D f)^{\star}(\tilde{g})$ at free maps $f_{0}: X \rightarrow Y$ that was from section 3.6.2 as well as Janet's equations from Weyl's Tube Formulas combined with Gauss's Theorema egregium.

Let $X=\left(X_{0} \times\left[0, t_{1}\right], g\right), t_{1}>0$, be a normally geodesically split Riemannian manifold which means that the lines $x \times\left[0, t_{1}\right]$ are geodesic that are normal to $X_{0} \times t$ for all $t \in\left[0, t_{1}\right]$.

Then the first and the second $t$-derivatives of the metrics $g(t)$ on $X_{0}$, that is our $g$ on $X$ restricted to $X_{0}=X_{t}=X_{0} \times t$, satisfy the following

\section{Weyl's Tube Formulas}

- The $t$-derivative of $g(t)$ equals the relative curvature, that is the second fundamental form of $X \times t \stackrel{I_{t}}{\subset} X$ denoted $K_{t}=K_{I_{t}}$,

$$
\partial_{t} g(t)=K_{t} .
$$

Write the quadratic (second fundamental) form $K_{t}$ as

$$
K_{t}(\tau, \tau)=\left\langle A_{t}(\tau), \tau\right\rangle_{g(t)}, \tau \in T\left(X_{t}\right),
$$

where $A_{t}: T\left(X_{t}\right) \rightarrow T\left(X_{t}\right)$ is what is called the shape operator of $X_{t} \subset X$.

\footnotetext{
${ }^{47}$ The essentiality of the Pythagorean inequality $K_{f_{0}}^{2}-K_{I}^{2}>0$ for extensions of local isometric immersions was emphasised in [4], where one can find references to older papers. Also this inequality enters the $h$-principle for the semilocal extensions; see 3.1.6 in 35 .

${ }^{48}$ Apparently, this, in the case of the $4 \mathrm{D}$-spacetime, was known to physicists since $\approx 1920$.
} 
Let $B_{t}(\tau, \tau)$ be the sectional curvature of $g(t)$ evaluated on the pairs $(\tau, \nu)$, where $\nu \in T(X)$ are unit vectors normal to $X_{t} \subset X$. Then the second derivative $\partial_{t t} g(t)$ is expressible in terms of this curvature and the square of the shape operator as follows:

$$
\partial_{t t} g(t)=B_{t}-A_{t}^{2}
$$

(See 37] for an elementary exposition of applications of these formulas in Riemannian geometry.)

If we now rewrite the definition of $f^{\prime}=M_{f_{0}}\left(g^{\prime}\right)$ via the linear equations from section 3.6.2 with $\partial_{t} f$ normal to $X_{t}$ instead of $f^{\prime}$ for $f: X=X_{0} \times\left[0, t_{1}\right] \rightarrow Y$ as

$$
\left\langle\partial_{t} f, \partial_{i j}^{2} f\right\rangle_{\tilde{g}}=-\frac{1}{2} \partial_{t} g_{i j}(t)
$$

where $\partial_{i}$ and $\partial_{i j}$ denote the derivatives in coordinates $x_{i}$ on $X_{0}=X_{t}$, we shall see that these directly follow from normality of $\partial_{t} f$ to $X_{t}$, that is $\left\langle\partial_{t} f, \partial_{i} f_{0}\right\rangle=0$, and item • combined with the (Pythagorean) Euler-Meusnier formula.

Similarly, by confronting item $\bullet$ with the Gauss formula, we obtain a system of linear equations for $\partial_{t t} f(t)$ expressed by the scalar products

$$
\left\langle\partial_{t t} f(t), \partial_{i j} f(t)\right\rangle_{\tilde{g}}=B_{i j}^{\circ}(f, g(t)),
$$

where $B_{i j}^{\circ}$ are certain expressions in the first derivatives of $f(t)$ and the first and the second derivatives of $g$.

Since $f_{0}$ is free, this system is non-singular for small $t>0$ and solvable in $\partial_{t t} f(t)$ for $f(t): X_{t} \rightarrow Y$.

It follows that the infinitesimal inversion is representable by a true evolution system of the second order which is solvable in the $C^{a n}$-case for small $t>0$ by the Cauchy-Kovalevskaya theorem, as was stated in section 3.7.

Finally, we differentiate the equations $\left\langle\partial_{t}, \partial_{t}\right\rangle_{\tilde{g}}=1$ and $\left\langle\partial_{t}, \partial_{i}\right\rangle_{\tilde{g}}=0$ that yield

$$
\left\langle\partial_{t t}, \partial_{t}\right\rangle_{\tilde{g}}=0
$$

and

$$
\left\langle\partial_{t t}, \partial_{i}\right\rangle_{\tilde{g}}=-\left\langle\partial_{t}, \partial_{i t}\right\rangle_{\tilde{g}}
$$

which together with $\left\langle\partial_{t t} f(t), \partial_{i j} f(t)\right\rangle_{\tilde{g}}=B_{i j}^{\circ}(f, g(t))$ compose the full Janet system, which, for free $f_{0}$, is solvable in $\partial_{t t} f(t)$ for small $t>0$ as we stated earlier in this section.

A more detailed analysis of Janet's equations yields the semilocal $h$-principle for free isometric $C^{r}$-extensions from smooth submanifolds $X_{0} \subset X$ to $X$, where the corresponding Nash process needs $r>5$ (see 3.1.6 in [35]), but it remains unclear what happens for $2<r \leq 549$

Another unsettled, seemingly easier, issue is to establish a sufficiently general semilocal $h$-principle for extensions of isometric immersions from singular, e.g., semianalytic, subsets $X_{0} \subset X$ to $X$ where (formulation of the problem in) the $C^{a n}$-case needs a bit of attention.

Why do we need the semilocal h-principle? Even if an isometric $C^{a n}$-immersion $f_{0}: X_{0} \rightarrow Y$ isometrically extends to (small) neighbourhoods $U_{x} \subset X$ of all $x \in X_{0}$, there may exist a topological obstruction for isometric $C^{2}$-extensions to open $U \supset X_{0}$.

\footnotetext{
${ }^{49}$ Possibly, Günther's perturbation scheme can lead to better $r$. But most apparently natural modifications of Nash do not fare well. For instance, the implicit function theorem used in [49] needs $r \geq 17$.
} 
The very formulation of the $h$-principle automatically incorporates all such obstructions and if it holds - and this is a BIG IF - this "principle" guarantees the existence of isometric extensions whenever such obstructions vanish.

For instance if a $C^{a n}$-submanifold $X_{0} \subset X$ is homeomorphic to the $m$-ball, then there is no such obstruction, and the corresponding $h$-principle (see 3.1.6. in [35]) tells you that

if $q \geq s_{n}=\frac{n(n+1)}{2}, n=\operatorname{dim}(X)$, then free isometric $C^{a n}$-immersions $f_{0}: X_{0} \rightarrow Y^{q}$ do extend to isometric $C^{a n}$-immersions $U \rightarrow Y^{q}$ of some open $U \supset X_{0}$.

On the other hand, if $X$ is the flat Möbius strip and $X_{0} \subset X$ is the central closed geodesic in it, then-this is obvious - free isometric immersions $f_{0}: X_{0} \rightarrow \mathbb{R}^{3}$, i.e., with non-vanishing curvatures, do not extend to neighbourhoods $U \subset X$ of $X_{0}$ unless the (one-dimensional) binormal bundle of $X_{0} \underset{f_{0}}{\subset} \mathbb{R}^{3}$ is non-orientable.

More generally, let $X_{0}=S^{n-1} /\{ \pm 1\}$ be the projective $(n-1)$-space, and let $X=S^{n-1} \times \mathbb{R} /\{ \pm 1\}$ be the total space of the canonical flat line bundle over $X_{0}$ with $X_{0}$ imbedded to $X$ as the zero section.

Let $f_{0}: X_{0} \rightarrow \mathbb{R}^{q_{0}}$ for $q_{0}=s_{n}-1, s_{n}=\frac{n(n+1)}{2}$, be the Veronese embedding, which is an isometric imbedding such that every isometry of $X_{0}$ extends to a linear isometry of $\mathbb{R}^{q_{0}} \underset{f_{0}}{\supset} X_{0}$. Compose $f_{0}$ with the standard embedding $\mathbb{R}^{q_{0}} \subset \mathbb{R}^{q}$ for $q=q_{0}+m$ and keep the same notation $f_{0}$ for the resulting composed map $X_{0} \rightarrow \mathbb{R}^{q}$.

If $m \geq 1$, then, obviously, $f_{0}$ locally $C^{\text {an }}$-extends to isometric maps $U_{x} \rightarrow \mathbb{R}^{q}$, for small open $U_{x} \ni x$ at all points $x \in X_{0}$. Also

all (moderately) $C^{2}$-small isometric $C^{a n}$-perturbations of $f_{0}: X_{0} \rightarrow \mathbb{R}^{q}, q>q_{0}$, are also extendable from $X_{0}$ to $U_{x}$ by the Janet-Burstin theorem.

But if $q=q_{0}+m \leq s_{n}+n-1$, then

no isometric $C^{2}$-extension of $f_{0}$ from $X_{0}$ to any neighbourhood $U \supset X_{0}$ exists.

Indeed, such an extension would send the $\mathbb{R}$-fibres in $X$ normally to the osculating spaces of $X_{0} \underset{f_{0}}{\subset} \mathbb{R}^{q}$, which is-this is topologically obvious-impossible for $q=$ $q_{0}+m \leq s_{n}+n-1$, where this non-extendability is stable under (moderately) small $C^{2}$-perturbations of $f_{0}$ and of the Riemannian metric in $X$.

On the other hand, by the semilocal version of Janet-Burstin, (moderately) small isometric $C^{a n}$-perturbations $f_{0}^{\prime}: X_{0} \rightarrow \mathbb{R}^{q}$ of $f_{0}$ admit isometric $C^{a n}$-extensions to certain neighbourhoods $U \supset X_{0}$ in $X$, provided $q=q_{0}+m \geq s_{n}+n, s_{n}=\frac{n(n+1)}{2}$, $n=\operatorname{dim}(X)$.

\section{QUESTIONS}

What are "maximally general" (robust?) sufficient conditions for extendability of not necessarily free isometric $C^{\text {an }}$-immersions from submanifolds $X_{0} \subset X$ to some neighbourhoods $U \supset X_{0}$ ?

Non-Extendability Example. The real analytic local Hopf-Schilt-Efimov immersions (see 3 in section 1.2) from surfaces $X$ to the 3 -space are "very flat" at $x_{0} \in X$. Albeit they are uniquely determined by their restrictions to curves $X_{0} \subset X$ that contain $x_{0}$, these are far from being free and Janet's extension lemma does not apply. In fact, no bending of such a curve extends to a bending of $X$ in $\mathbb{R}^{3}$.

67 Can anything comparable happen to (local germs of) manifolds $X \subset \mathbb{R}^{q}, q=s_{n}=$ $\frac{n(n+1)}{2}$, of dimensions $n>2$ and their submanifolds $X_{0} \subset X$ ? 
What, on the contrary, is the full set of (semi)algebraic constraints on the $r$ th derivatives of an isometric $C^{\text {an }}$-immersion $f_{0}: X_{0} \rightarrow \mathbb{R}^{q}$ at a point $x_{0} \in X_{0}$, such that $f_{0}$ isometrically $C^{\text {an }}$-extends to a neighbourhood of $x_{0} \in X$ ?

(Such a condition may be easier to identify if one requires that also all small isometric perturbations $f_{0}^{\prime}$ of $f_{0}$ extend to $X$.)

For instance,

what are conditions for an isometric $C^{\text {an }}$-immersion $X_{0} \rightarrow \mathbb{R}^{q}$ to be locally extendable to an isometric $C^{a n}$-immersion of the Riemannian product $X=X_{0} \times \mathbb{R}^{k}$ ?

It is plausible that such an extension of isometric $C^{a n}$-immersions $f_{0}: X_{0} \rightarrow \mathbb{R}^{q}$ from $X_{0}$ to the ambient $X=X_{0} \times \mathbb{R}^{k}$ is always (very often?) possible if $\operatorname{dim} X_{0}=1$ and $q \geq s_{n}=\frac{n(n+1)}{2}, n=\operatorname{dim}(X)$, (isometric $C^{\infty}$-immersions $X_{0} \rightarrow \mathbb{R}^{q}$ do not always admit local isometric $C^{2}$-extensions to $\left.X\right)$, but there may be robust obstructions for extensibility of $f_{0}$ from $X_{0}$ to $X \supset X_{0}$ for $\operatorname{dim}\left(X_{0}\right) \geq 2$.

In fact, the differential $D f$ of an isometric $C^{2}$-map $f: X=X_{0} \times \mathbb{R}^{k} \rightarrow \mathbb{R}^{q}$ sends the the tangent spaces of the $\mathbb{R}^{k}$-fibres of $X$ normally to the (second) osculating spaces of $X_{0}=X_{0} \times 0 \underset{f}{\subset} \mathbb{R}^{q}$. If $\operatorname{dim}(X) \geq 2$ and if the dimension of the osculating space drops down at a point $x_{0} \in X_{0}$, then the (rational-like) map $D f$ may undergo a blow-up at $x_{0} \in X_{0}$.

\subsection{Addition of $d \phi^{2}$ by $C^{\infty}$-Twist, Semilocal Immersions, and Global Cylinders.}

The existence of isometric $C^{r}$-extensions of immersions $f: X \rightarrow Y$ from $X=$ $X \times 0 \subset X \times D^{2}(\varepsilon)$ to $X \times D^{2}(\varepsilon)$, where $D^{2}(\varepsilon)$ is the $\varepsilon$-disk with arbitarily small, yet positive radius, allows addition of differentials of $C^{r}$-functions $\phi: X \rightarrow \mathbb{R}$ to the metrics induced by $f$ by means of isometric maps $\mathbb{R} \rightarrow D^{2}(\varepsilon)$.

In fact, one only needs such extensions on small neighbourhoods $U \subset X$ and also $C^{r}$-approximately isometric extensions often suffice (see 3.1.2 and 3.1.7 in [35]). Thus one shows that

[ם] $C^{r}$-smooth free isometric immersions $f: X^{n} \rightarrow Y^{q}$ for $r>4$ and $q \geq s_{n+2}=$ $\frac{(n+2)(n+3)}{2}$ satisfy the $h$-principle,

where, we recall, $s_{n+2}$ is the minimal dimension allowing $C^{\infty}$-immersions of $(n+$ 2)-dimensional manifolds, such as $X \times D^{2}(\varepsilon)$.

CONJECTUREs. It is plausible that this kind of construction works for free maps $X^{n} \rightarrow Y^{q}$ for all $q \geq s_{n+1}=s_{n}+n 50$

If so then, most likely, one will be able to construct strata-wise free, with respect some stratification of $X^{n}$, isometric $C^{\infty}$ - and $C^{a n}$-immersions of all $X^{n}$ to $\mathbb{R}^{s_{n+1}}$.

On the other hand, there is not even a hint of a possible construction if $q<s_{n+1}$.

Turning Semilocal. The lower bound $q \geq s_{n+2}$ can be improved for semilocal isometric immersions that are isometric immersions $U \rightarrow Y$ from a (possibly very small) neighbourhood $U \subset X$ of a given $X_{0} \subset X$.

If $X_{0}$ is a $C^{\infty}$-submanifold with $\operatorname{codim}\left(X_{0}\right) \geq 2$, then such free isometric maps $U \rightarrow Y$ from a unspecifiably small $U \supset X_{0}$ satisfy the $h$-principle with no restriction on $q=\operatorname{dim}(Y)$. This follows from the above $h$-principles for free isometric immersions $X_{0} \rightarrow Y$ and that for semilocal extensions from the previous section.

Moreover, in the $C^{a n}$-case there is a version of the semilocal $h$-principle for not necessarily free isometric immersions.

\footnotetext{
${ }^{50}$ Günther succeeds in doing this for $q \geq s_{n+1}+4$ but, alas, I am unable to follow his argument.
} 
For instance, let the manifolds $X^{n}$ and $Y^{q}$ be parallelisable, and let $X_{0}$ have trivial normal bundle in $X^{n}$.

[ㄱ] If $q \geq s_{n}=\frac{n(n+1)}{2}$, then every strictly curve-shortening map $f_{0}: X_{0} \rightarrow Y^{q}$ can be approximated by free isometric $C^{a n}$-maps $f_{1}: X_{0} \rightarrow Y^{q}$ that admit isometric $C^{a n}$-extensions to small neighbourhoods $U \supset X_{0}$ in $X$ (that depend on $f_{1}$ ).

71 Conjecture. Probably it is not hard to extend this result to a large class of (all?) semianalytic subsets $X_{0} \subset X$, e.g., $m$-skeleta of analytic triangulations of $X$ and $m<n=\operatorname{dim} X$.

But it seems harder to do this for $\operatorname{codim}\left(X_{0}\right)=1$ without an additional lower bound on $q$ or of strong restriction on the induced metric on $X_{0}$, since isometric immersions $X^{n} \rightarrow Y^{q}$, say for $q=s_{n+1}$, remain unavailable for general manifolds $X_{0}$.

Cylinders and Tori. There are particular classes of metrics on $X^{n}$ that go to $Y^{q}$ starting from $q=s_{n+1}$.

For instance, let $X_{0}=\left(X_{0}, g\right)$ and $Y=(Y, \tilde{g})$ be compact Riemannian $C^{a n}$ manifolds, and let $f_{0}, f_{2}=1: X_{0} \rightarrow Y$ be free isometric $C^{\infty}$-immersions that can be joined by a homotopy of $C^{1}$-maps $f_{t}: X_{0} \rightarrow Y$ that strictly decreases the lengths of all curves in $X_{0}$. Then a "fold+twist" construction from 3.1.8 in [35] in the spirit of Kuiper's "smoothing the corners" (see section 3.3) delivers the following immersions $F$ of (parts of) $m$-dimensional cylinders $X=X_{0} \times \mathbb{R}(m=n+1)$ to $Y^{q}$ for $q=s_{m+1}=\frac{(m+1)(m+2)}{2}=s_{m}+m+1$.

If $Y$ is parallelisable and $q=\operatorname{dim}(Y) \geq s_{n+2}$ for $n=\operatorname{dim}\left(X_{0}\right)$, then there exits an isometric $C^{\infty}$-map $F:\left(X_{0} \times\left[0, t_{2}\right], g+d t^{2}\right) \rightarrow Y$ for some (large) $t_{2}>0$, such that $F_{\mid X_{0} \times 0}=f_{0}$ and $F_{\mid X_{0} \times t_{2}}=f_{2}$.

For example, two $C^{\infty}$-smooth free (i.e., with non-vanishing curvatures) closed curves in $\mathbb{R}^{q}$ of equal length can be joined by an isometrically immersed $C^{\infty}$ cylinder, provided $q \geq 6$, but this is unknown for $q=4,5$. (The constructions of isometric immersions of closed surfaces to $\mathbb{R}^{5}$ in 3.2 .4 in 35 may apply to here for $q=5$.)

Similarly to cylinders, one constructs isometric immersions of manifolds that locally split along some submanifolds in them. For example

every strictly curve-shortening map from a flat (not necessarily split!) $n$-torus to a parallelisable51 Riemannian $C^{\text {an }}$-manifold $Y^{q}, q \geq s_{n+1}$, can be approximated by isometric $C^{a n}$-immersions.

Probably, generic Riemannian $C^{\infty}$-manifolds $Y^{q}$ with $q<s_{n}$ receive no isometric $C^{\infty}$-immersions $f$ from flat manifolds $X^{n}$, but one may expect plenty of such $f$ if $Y^{q}$ is also flat even for relatively small $q<<s_{n}$, where "plenty" means density of these $f$ in the space of strictly curve-shortening maps $X \rightarrow Y$.

Apparently, there is no known obstruction for this if $q \geq 2 n$ and not even any conceivable one for $q \geq 3 n$.

And one has not the vaguest idea concerning minimal $q$, such that every flat (non-split) $n$-torus $C^{r}$-isometrically, $r \geq 2$, embeds to $\mathbb{R}^{q}$.

72 What is the full range of " $t$ wist constructions" where the global existence problem for structure inducing maps is reduced to a semilocal extension problem?

73 When can the "twist" be replaced by "stretching+smoothing the corners", thus improving the bound on the dimensions of ambient spaces?

\footnotetext{
${ }^{51} \mathrm{I}$ am uncertain if this is truly needed here.
} 
Stretching Tori in Euclidean Spaces. Let us construct isometric real analytic immersions of flat $n$-tori $X=X^{n}$ to $\mathbb{R}^{q}$ for $q=s_{n}+\frac{n}{2}$ for $n$ even and $q=s_{n}+\frac{n-1}{2}$ for $n$ odd.

This is achieved by performing $q-2 n$ consecutive stretchings $X_{i} \leadsto X_{i+1}$ applied to flat tori $X_{i} \subset \mathbb{R}^{2 n+i}, i=0,1, \ldots, q-2 n-1$, starting from $X_{0} \subset \mathbb{R}^{2 n}$ and terminating with $X_{q-2 n}=X \subset \mathbb{R}^{q}$, where

- each $X_{i+1}$ lies over $X_{i}$,

$$
X_{i+1} \subset X_{i} \times \mathbb{R}=p_{i}^{-1}\left(X_{i}\right) \subset \mathbb{R}^{2 n+i+1}=\mathbb{R}^{2 n+i} \times \mathbb{R} \stackrel{p_{i}}{\rightarrow} \mathbb{R}^{2 n+i} \supset X_{i} ;
$$

- the submanifold $X_{i+1} \subset X_{i} \times \mathbb{R}$ is equal to the graph of a $C^{a n}$-function $\phi_{i}: X_{i} \rightarrow \mathbb{R}$ all levels $\phi^{-1}(y) \subset X_{i}, y \in \mathbb{R}$, of which are flat subtori in $X_{i}$ of codimension one.

By linear algebra, an arbitrary flat metric on the torus can be obtained by $s_{n}-n$ such stretchings from a split metric and, since split $n$ tori embed to $\mathbb{R}^{2 n}$, all flat tori embed to $\mathbb{R}^{q}$ for $q=s_{n}-n+2 n=s_{n}+n$.

Now, to gain $n / 2$, we observe (this goes back to Blanusa) that all flat 2-tori $X^{2}$ isometrically immerse to the $r$-spheres $S^{3}(r) \subset \mathbb{R}^{4}, r=r(X)>0$, as pull-backs of immersed closed curves in $S^{2}$ under the Hopf fibration $S^{3}(r) \rightarrow S^{2}(r)$.

And if we start with a torus $X_{0}$ split to arbitrary, non-split(!) 2-subtori (and a circle if $n$ is odd), we shall need $s_{n}-3 n / 2$, rather than $s_{n}-n$ stretchings to bring $X_{0} \sim X$, since non-split 2-tori depend on three independent parameters rather than two as split 2-tori do, where this parameter counting argument is easily justifiable by linear algebra. The odd case is handled similarly and the proof follows.

\section{Production of Problems and Classification of Structures}

Formulating problems is, probabaly, the most essential aspect of human mathematics; for this reason, mathematicians have been shying away from a mathematical study of this process. Breaking the tradition, let us try to do this in the context of induced geometric structures.

There are three essential "parameters" that structure inducing problems depend on.

1. Geometric Categories $\mathcal{X}$. Objects of our geometric categories are "spaces" $X$ where morphisms $f \in \mathcal{X}$ are maps $f: X \rightarrow Y$.

2. Classes $\mathcal{G}$ of Structures. Objects from $\mathcal{X}$ may be endowed with "geometric structures" from a certain class $\mathcal{G}$.

Then morphisms, $f \in \mathcal{X}$ that are maps $f: X \rightarrow Y$, (may) induce such structures $g$ on $X$ from structures $h$ on $Y$, written as $g=f^{\star}(h)$.

The spaces from $\mathcal{X}$ augmented with structures from $\mathcal{G}$ and the structure preserving maps $f:(X, g) \rightarrow(Y, h)$ make a new category, say $\mathcal{G}[\mathcal{X}]$, the morphisms that we call $\mathcal{G}$-isometric maps. Such maps and categories $\mathcal{G}[\mathcal{X}]$ of these are the primary subject matter of our study.

Abstractly, classes $\mathcal{G}$ can be defined as transformations of categories

$$
\mathcal{X} \sim \mathcal{G}[\mathcal{X}]
$$

3. Classes $\mathcal{I}$ of Invariants of Structures, of Spaces of Maps, and of Structure Inducing Operators. Such invariants express the properties of spaces, maps, and structures under study. They may be used for classification of categories $\mathcal{X}$ and classes $\mathcal{G}$.

Also, isolating/defining such invariants serves to articulate the questions we ask. 
For instance an analyst would ask what is the topology of the subspace $M_{g \rightarrow h}$ of structure inducing maps $(X, g) \rightarrow(Y, h)$ in the space $M_{X \rightarrow Y}$ of all maps $X \rightarrow Y$ in $\mathcal{X}$ :

under what conditions is $M_{g \rightarrow h}$ non-empty? When is it closed, open, or dense in $M_{X \rightarrow Y}$ ? When is the structure inducing operator $f \mapsto g=f^{\star}(h)$ injective, surjective, or open?

And an algebraic topologist will be concerned with the homotopy types of these spaces:

under what conditions is the inclusion

$$
M_{g \rightarrow h} \subset M_{X \rightarrow Y}
$$

k-connected? What, in general, is the (co)homology homomorphism induced by this inclusion? When does the structure inducing operator have the Serre (or a weaker one) homotopy lifting property?

Since $\mathbf{1}, \mathbf{2}$, and $\mathbf{3}$ above are essentially independent, they serve as coordinates in the "space of questions": by picking up particular $\mathcal{X}, \mathcal{G}, \mathcal{I}$ at will, we generate questions of the following kind.

A. What are the "values" of the $\mathcal{I}$-invariants of $\mathcal{G}[\mathcal{X}]$ ?

B. What are the cases where suitably generalised Nash methods enable one to, at least partially, answer $\boldsymbol{A}$ ?

C. What are the conjectural answers to questions from $\boldsymbol{A}$ and the conceivable approaches to prove/disprove such conjectures when the Nash-style methods refuse to apply?

With all this in the back of one's mind, the study of induced structures must be preceded by the description/enlisting of relevant categories and their invariants.

Besides, one has to account for the cases that do not fit to the above framework.

For instance, the tensorial equation $g=\sum_{i}\left(d \psi_{i}\right)^{p}$, where the unknowns are exterior differential forms $\psi_{i}$ of degrees $l>0$ on a manifold $X$, is not directly associated with maps between manifolds. Yet, the corresponding

differential operator $\left\{\psi_{i}\right\} \mapsto g=\sum_{i}\left(d \psi_{i}\right)^{p}$ does commute with smooth maps $X_{1} \rightarrow X_{2}$.

\subsection{Categories, Sheaves, Jets, and $h$-Principles.}

In analysis of non-linear PDEs the basic categories are those of $C^{r}$-maps $f: X \rightarrow$ $Y$ between smooth manifolds where $r$ may stand for Hölder $(r, \alpha)$.

Categories of this kind carry extra structures within themselves. For instance, sets of morphisms map $(X \rightarrow Y)$ are topological spaces. Thus one may speak of continuous families of maps $f_{p}: X \rightarrow Y$ parametrised by some $P \ni p$.

Continuous Subpolyhedral Spaces. In fact, we often do not care about the topological structure in $\operatorname{map}(X \rightarrow Y)$ per se but only in such families $f_{p}$ for polyhedral spaces $P$.

The structure defined in an "abstract space" $M$ by such families that satisfy a list of obvious properties is sometimes called a "quasi-topology" which is essentially the same as the semisimplicial structure. We prefer to call it a continuous subpolyhedral structure.

This structure, unlike the topological one, survives inductive limits.

For instance, if $X_{0} \subset X$ is a closed subset, then the space of germs of $C^{r}$-maps $X \rightarrow Y$ at $X_{0}$ (that is, the inductive limit of the spaces of maps $U \rightarrow Y$ for arbitrarily 
small neighbourhoods $U \subset X$ of $X_{0}$ ) come with a natural continuous subpolyhedral structure.

We assign such continuous subpolyhedral structures to the (topological) spaces of maps, $\operatorname{map}(U \rightarrow Y)$ for all open subsets $U \subset X$, and we observe that these spaces and the obvious arrows

$$
\operatorname{map}\left(U_{1} \rightarrow Y\right) \nrightarrow \operatorname{map}\left(U_{2} \rightarrow Y\right), U_{2} \subset U_{1},
$$

define a sheaf over $X$, called a continous subpolyhedral sheaf of maps $X \rightarrow Y$, denoted $\Phi=\operatorname{sheaf}(X \rightarrow Y)$, where $\Phi(U)=\operatorname{map}(U \rightarrow Y), U \subset X$.

This allows continuous disassembly of categories of structure preserving maps by introducing new "disassembled" morphisms as continuous families of germs of maps $\left(U_{x}, x\right) \rightarrow\left(V_{y}, y\right)$ for "infinitely small" neighbourhoods $U_{x} \subset X$ and $V_{y} \subset Y$ of points $x \in X$ and $y \in Y$.

The disassembly defines a continuous, obviously injective, morphism between sheafs over $X$,

$$
\text { disas : } \Phi \longrightarrow \Psi=\Phi_{\text {germ }},
$$

where in the present case $\Phi=\operatorname{sheaf}(X \rightarrow Y)$ and $\Psi=\operatorname{sheaf}_{\text {germ }}(X \rightarrow Y)$ and where, we recall, morphisms between sheaves over $X$ are given by continous maps $\Phi(U) \rightarrow \Psi(U), U \subset X$.

"Abstract h-Principle". A property of continuous disassembly (disas) we are particularly intersted in is it being a weak homotopy equivalence, which means weak homotopy equivalence of the maps $\Phi(U) \rightarrow \Psi(U)$ for all open $U \subset X$, where "weak" signifies that "topology" is downgraded to continuous subpolyhedral structure.

Intuitively, this means that, on the homotopy level, true maps $X \rightarrow Y$ can be assembled from continuous families of germs, $U_{x} \rightarrow V_{y}$.

Such a weak homotopy equivalence is obvious as far as all maps $X \rightarrow Y$ are concerned, but it is by no means automatic in the categories of $\mathcal{G}$-isometric maps: if it holds at all, it constitutes the essential geometric core of what we call the $h$-principle for these maps.

One can restrict categories of $C^{r}$-maps without changing $\mathcal{G}$, e.g., by taking symplectic or contact immersion that additionally is required to be $\mathcal{G}$ isometric.

But algebraically defined classes $\mathcal{G}$ of structures, e.g., of quadratic differential (forget "positive definite"), adapt to different categories in a chameleon-like fashion by changing their "colours" but keeping their "souls" intact.

Thus, one may speak of $\mathcal{G}$-isometric maps in the following categories:

(an) complex analytic manifolds (spaces?) and holomorphic maps;

(alg) algebraic manifolds over some field $\mathbb{F}$ and regular maps;

$\left(\mathbf{c}_{\infty}\right)$ linear spaces over $\mathbb{F}$ and "formal maps" between them defined with formal power series on these spaces;

$\left(\mathbf{c}_{r}\right)$ linear spaces over $\mathbb{F}$ and non-homogeneous polynomial maps of degree $r$ that send $0 \mapsto 0$.

These $\left(\mathbf{c}_{r}\right)$, in the case $\mathbb{F}=\mathbb{R}$, can be coupled with topological categories, where an instance of such coupling is the category of vector bundles and fibre preserving and fibrewise linear maps.

This category, call it $\mathcal{V} B_{1}$, goes along with the category, $\mathcal{C}_{\text {germ }}^{1}$ of smooth manifolds and continuous families of germs of $C^{1}$-maps, where the differentials at the points $x \in X$ transform $\mathcal{V} B_{1} \sim \mathcal{C}_{\text {germ }}^{1}$ by reducing $C^{1}$-germs $U_{x} \rightarrow U_{y}$ to linear maps between tangent spaces $T_{x} \rightarrow T_{y}$. 
Similarly, the $r$-jets of $C^{r}$-maps $f: U_{x} \rightarrow U_{y}$ for $r=1,2, \ldots, \infty$, transform/reduce $C^{r}$-germs to $r$-jets of maps that can be represented, albeit non-canonically 52 by polynomial maps $T_{x}(X) \rightarrow T_{y}(Y)$ of degree $r$ for $r<\infty$ and by such "formal maps" for $r=\infty$.

To be canonical, one defines the $r$ th tangent bundle $T^{[r]}(X)$ similarly to the tangent bundle $T(X)=T^{[1]}(X)$ as the linear dual to the vector bundle of $r$-jets of germs of smooth functions on $X$ modulo constants.

Notice that the fibre $T_{x}^{[r]}(X)$ at $x \in X$ is equal to the linear span of the cones $V_{x} \subset T_{x}^{[r]}(X)$ of $r$-jets of of smooth curves issuing from $h$ which are smooth maps $\mathbb{R} \rightarrow X, 0 \mapsto x$.

Filtration on the Jets. The bundle $T_{x}^{[r]}(X)$ is naturally filtered by the inclusions

$$
T(X)=T^{[1]}(X) \subset T^{[2]}(X) \subset \cdots \subset T^{[r]}(X) \subset \cdots,
$$

where the quotient bundle $T^{[r]}(X) / T^{[r-1]}(X)$ is, for all $r$, canonically isomorphic to the $r$ th symmetric power of the tangent bundle $T(X)$.

And, thinking of the $r$-jet as "the full differential of order $r$ ", one denotes the $r$-jets of smooth maps $X \rightarrow Y$ by

$$
D^{[r]}: T^{[r]}(X) \rightarrow T^{[r]}(Y)
$$

that are fibrewise linear maps between the bundles of jets 53

Jets and the $h$-Principle. Identification of the spaces $T_{x}^{[r]}(X)$ with the dual to the spaces of polynomials on $T_{x}(X)$ allows one to speak of the $h$-principle for $\mathcal{G}$-isometric $C^{r}$-maps as the weak homotopy equivalence property of the $r$ th jet regarded as a map from the space of $\mathcal{G}$-isometric $C^{r}$-maps $X \rightarrow Y$ to the space of continuous fibrewise polynomial $\mathcal{G}$-isometric maps $T(X) \rightarrow T(Y)$.

Here, a priori, the validity of this property depends on how one identifies linear maps $T_{x}^{[r]}(X) \rightarrow T_{y}^{[r]}(Y)$ with polynomial maps $T_{x}(X) \rightarrow T_{y}(Y)$. But since the space of such identifications is, clearly, contractible, this does not affect the homotopy types of our spaces.

Also there is an uncertainty in the definition of $\mathcal{G}$-isometric $r$-jets for $r<\infty$, since high derivatives of $\mathcal{G}$-isometric maps may influence the lower ones, as happens in the Gauss curvature formula in the case of Riemannian isometric immersions.

An obvious space between $\operatorname{sheaf}(X \rightarrow Y)$ and $\operatorname{sheaf}_{\text {jet }}\left(T^{[r]}(X) \rightarrow T^{[r]}(Y)\right)$ is that of continuous families of germs sheaf $\operatorname{germ}(X \rightarrow Y)$. Thus the proof of the $h$ principle would follows from the above abstract $h$-principle and the local $h$-principle that the homotopy equivalence of the jet-map for germs,

$$
\text { JET }: \text { sheaf }_{\text {germ }}(X \rightarrow Y) \rightarrow \operatorname{sheaf}_{\text {jet }}\left(T^{[r]}(X) \rightarrow T^{[r]}(Y)\right) .
$$

Proving or disproving this local $h$-principle may be difficult, but unlike the "abstract $h$-principle" 54 this is a matter of local analysis of the differential equations imposed on germs of maps by the $\mathcal{G}$-isometry condition.

\footnotetext{
${ }^{52}$ The $r$-jet at the origin $0 \in \mathbb{R}^{n}$ of a smooth function $\mathbb{R}^{n} \rightarrow \mathbb{R}$ is represented by its $r$ th Taylor polynomial. But non-linear changes of coordinates preserve only the filtration by degree, not the polynomial grading of the Taylor polynomials.

${ }^{53}$ Another possible device to keep the track of all derivatives of orders $\leq r$ is the iterated tangent bundle $T(T(\ldots(T(X) \ldots)))$, but this is more wasteful than $T^{[r]}$.

${ }^{54}$ The proof of this "abstract principle" in all known cases has been obtained by means of geometric constructions. Also, the rare significant successes in disproving it (only in three cases?) have also been achieved by introducing geometric ideas.
} 
Amazingly, in contrast with examples of formally solvable but unsolvable linear PDEs with polynomial coefficients and with $C^{\infty}$-smooth right-hand sides that go back to Hans Lewy,

there is no single known counterexample to the local h-principle for (truly)

non-linear equations in the $C^{\infty}$-category.

(Saying "local $h$-principle" rather than "local solvability" rules out equations that are formally unsolvable. But there also exist $C^{a n}$-equations that are formally but not $C^{a n}$-solvable. Probably, the local solvability problem makes sense only for "sufficiently generic" non-linear equations that harbor no algebraic singularities in them.)

A particularly extensively studied case is that of local isometric immersions of surfaces to 3 -space, where there are many local solvability/non-solvability results (see [46, 44, 61])55 Also it is known 9] that generic $C^{\infty}$-metrics on 3-manifolds are locally $C^{\infty}$-immersible to $\mathbb{R}^{6}$, but no high regularity counterexamples are known.

Topological Obstructions to Isometric Immersions. The $h$-principle for $C^{r}$ smooth isometric immersions $X \rightarrow Y$ says, in particular, that the only obstruction to the existence of such an immersion is non-existence of a "fibrewise isometric" map between the jet spaces $T^{[r]}(X) \rightarrow T^{[r]}(Y)$ that represents the $r$-jet of a continuous in $x \in X$ family of infinitesimally (of order $r$ at $x$ ) isometric $C^{r}$-maps $X \subset U_{x} \rightarrow U_{y} \supset$ $Y$, where $x \mapsto y$ and where $U_{x}$ and $U_{y}$ are "infinitesimally small" neighbourhoods of $x \in X$ and of $y \in Y$.

Unlike the $h$-principle, it is obvious that topological obstructions for the existence of "fibrewise isometric" maps $T^{[r]}(X) \rightarrow T^{[r]}(Y)$ automatically obstruct isometric immersions $X \rightarrow Y$. But the existence/non-existence of such an obstruction for given $X=(X, g)$ and $Y=(Y, h)$ is a non-trivial problem in algebraic topology that needs for its solution a preliminary algebraic analysis of individual spaces of "isometric" maps (formal for $r=\infty) T_{x}^{[r]}(X) \rightarrow T_{y}^{[r]}(Y)$ at all points $x \in X$.

It is possible but improbable that there are these kinds of topological obstructions that would be applicable to $C^{r}$-isometric immersions $X^{n} \rightarrow \mathbb{R}^{q}$ for $q \geq s_{n}=n(n+1) 2$ and large $n$, say $n>3$, but such obstructions must(?) be significant for higher dimensional families of jets of local isometric immersions.

Namely, let $\mathcal{X} \rightarrow B$ be a $C^{a n}$-vector bundle, where $B$ is identified with the zero section $\mathbf{0} \subset \mathcal{X}$ and where the fibres are denoted $X_{b} \subset \mathcal{X}, b \in B$.

Given a Riemannian $C^{\infty}$-metric $G$ on $\mathcal{X}$, a $C^{r+1}$-map $F: \mathcal{X} \rightarrow \mathbb{R}^{q}$ is called $r$ infinitesimally fibrewise isometric if the metrics induced on the fibres $X_{b}$ at the points $b=\mathbf{0}_{b} \in X_{b}$ coincide with $G_{\mid X_{b}}$ with their derivatives up to order $r$.

Question A. What is the minimal $q=q(\mathcal{X}, r)$ such that $\mathcal{X}$ admits fibrewise $r$-infinitesimally isometric map $\mathcal{X} \rightarrow \mathbb{R}^{q}$ for all $G$ on $\mathcal{X}$ ?

QUESTION B. What is the minimal $q=q(n, d)$ such that all $n$-dimensional vector bundles $\mathcal{X}$ over $d$-dimensional $B$ admit fibrewise $r$-infinitesimally isometric maps to $\mathbb{R}^{q}$ for all metrics $G$ on $\mathcal{X}$ and all $r$ ?

The main difficulty in answering these questions is due to possible singularities in the spaces of jets of isometric maps, such as those exhibited by the Hopf-Schilt surfaces.

${ }^{55}$ This contains a corrected version of [60]. 
But singularities are absent from the spaces of jets of free isometric maps, which allow a satisfactory answer in this case and an upper bound on $q$ in general (see 3.3.1 in [35]). For instance,

if the bundle $\mathcal{X} \rightarrow B$ is trivial, then $q=q(\mathcal{X}, r)$ is bounded by $s_{n}=\frac{n(n+1)}{2}$, $n=\operatorname{dim} X_{b}$ for all $r$ with the equality $q=s_{n}$ for all sufficiently large $r \geq r(n)$, and in the case $B$ the dimension $q=q(n, d)$ is at most $s_{n}+n+d$.

Then, whenever the $h$-principle holds, one obtains, say in the context of Question A, an isometric immersion of parallelisable Riemannian $n$-manifolds to $\mathbb{R}^{q}$. But unfortunately, this $h$-principle is unknown for the most interesting $q \approx s_{n}$.

\subsection{Trends in Structures.}

Riemannian structures on manifolds $X$ are (functorially) characterised by the sets of isometric immersions $[a, b] \rightarrow X$. In infinitesimal terms this reads as follows.

Inherently One Dimensional Structures. An inherently one dimensional structure $g$ of order $l$ on a smooth manifold $X$ is a locally closed subset in the $l$ th jet bundle of $X$, denoted

$$
S(g) \subset T^{[l]}(X),
$$

such that fibres

$$
S_{x}(g)=S(g) \cap T_{x}^{[l]}(X) \subset T_{x}^{[l]}(X) \subset T^{[l]}(X), x \in X,
$$

are semialgebraic subsets and where this $S(g)$ is decomposed to a finite union of semialgebraic subsets carrying "identification marks" on them.

Examples. (a) Riemannian structures $g$ come this way via the associated unit sphere subbundles

$$
S(g)=\{\tau\}_{\|\tau\|_{g}=1} \subset T(X) .
$$

Here no marking is needed - there is only a single mark on $S(g) 56$

But $k$-tuples of Riemannin metrics 57 require $k$-different marks on the corresponding subbundles spheres in $T(X)$.

Also, if a single $g$ is pseudo-Riemannin, then the set $S(g)=\{\tau\}_{\|\tau\|_{g}= \pm 1}$ naturally caries two different \pm marks on it.

And symmetric differential form $g$ of all degrees $p$ are similarly defined by triply marked $S(g) \subset T(X)$ that are the sets of vectors $\tau \in T(X)$, where $g(\tau, \ldots, \tau)=0, \pm 1$.

(b) One also finds among our structures (unmarked) vector subbundles $\Theta \subset T(X)$ and also $\Theta$ accompanied by forms $g$, e.g., Riemannian metrics, on them, where the latter are called Carnot-Carathéodory structures 58

(c) Riemannian metrics of order $l>1$ are also pretty looking. These are families $g$ of Euclidean metrics $g_{x}$ in the fibres $T_{x}^{[l]}(X)$, where particular instances of these are associated to the ordinary Riemannian structures, as we shall see below.

\footnotetext{
${ }^{56}$ Whenever only one mark is involved, we do not mention marking at all.

${ }^{57}$ Isometric immersions of these for $k=2$ were studied 18 .

${ }^{58}$ Isometric immersions for these are studied in [17] and [18. Also the Nash implicit function theorem for Carnot-Carathéodory manifolds was used in 38 for an evaluation of Dehn functions in nilpotent groups.
} 
Given a smooth map $f: X \rightarrow(Y, h)$, one defines the induced structure $g=$ $\left(D^{[l]} f\right)^{\star}(h)$ on $X$, which is a subset $S(g) \subset T^{[l]}(X)$, as the pull-back

$$
S(g)=\left(D^{[l]} f\right)^{-1}(S(h)) \text { for } D^{[l]} f: T^{[l]}(X) \rightarrow T^{[l]}(Y) \supset S(h),
$$

with the marks on $S(g)$ coming from those on $S(h)$.

"Isometry" for a $C^{l}$-map $f:(X, g) \rightarrow(Y, h)$ may have two meanings:

- Isometric: the $r$ th jet $D^{[r]} f: T^{[l]}(X) \rightarrow T^{[l]}(Y)$ sends $S(g) \rightarrow S(h) \subset T^{[l]}(Y)$ by a marking preserving map;

- Strictly Isometric: the structure $g$ is induced by $f$ from $h$ that is $S(g)=$ $\left(D^{[l]} f\right)^{-1}(S(h))$.

In some cases, e.g., for Riemannian structures,

"isometric" $\Leftrightarrow$ "strictly isometric",

but, for instance, isometric immersions between Carnot-Carathéodory spaces are not necessarily strictly isometric.

ConjeCtuRE. Most (all?) inherently one dimensional structures are amenable to the techniques presented in section 3, since our main constructions are "inherently one dimensional", such as oscillatory curves and by $C^{\perp}$-approximation.

Isometric Immersions of Higher Order. The $r$ th jets $D^{[l]}(f): T^{[l]}(X) \rightarrow$ $T^{[l]}(Y)$ of $C^{r \geq l}$-smooth maps $f: X \rightarrow Y$ induce quadratic forms on the bundle $T^{[l]}(X)$, here we call them $G$, from quadratic forms $H$ on $T^{[l]}(Y)$, where the correspondence $f \mapsto G=\left(D^{[l]} f\right)^{\star}(H)$ is a (quadratic) differential operator of order $r$.

This operator for all $l$ looks very much is similar to that for $l=1$ corresponding to the ordinary isometric immersions and some (all?) results have, probably, their counterparts for all $l$.

Solutions of this general $r$ th order isometric immersions equation can be, probably, obtained similarly to how Nash does it for $r=1$, but we look below at a special case of these.

Assume $Y=\mathbb{R}^{q}$, and observe that the linear dual to the representation of the cotangent bundle of $X$ by linear functions on $\mathbb{R}^{q}$ defines a natural homomorphism that depends on flat affine structure in $\mathbb{R}^{q}$,

$$
T^{[l]}\left(\mathbb{R}^{q}\right) \rightarrow T\left(\mathbb{R}^{q}\right) .
$$

(Such a map can be constructed via covariant differentiation in an arbitrary Riemannian manifold $Y$, but I feel uncomfortable with this definition for $l>2$.)

Definition of $H$ on $\mathbb{R}^{q}$. Let $H$ be the (positive semidefinite) form on $T^{[l]}\left(\mathbb{R}^{q}\right)$ induced by this homomorphism from the form $\sum_{j} d y_{j}^{2}$ on $\mathbb{R}^{q}$.

Symmetrisation of $G$ on $X$. Recall the jet filtration from the previous section,

$$
T(X)=T^{[1]}(X) \subset T^{[2]}(X) \subset \cdots \subset T^{[k]}(X) \subset \cdots \subset T^{[l]}(X),
$$

where $T^{k}=T^{[k]}(X) / T^{[k-1]}(X)$ are canonically isomorphic to the $k$ th symmetric powers of the tangent bundle $T(X)$.

Given a quadratic form $G$ on $T^{[l]}(X)$, define a "fully symmetric" quadratic form on $\oplus_{k} T^{k}(X)$ that is associated to it as

$$
\operatorname{sym}_{\oplus} G=\bigoplus_{k}^{l} \operatorname{sym} \underline{G}_{k},
$$


where $\underline{G}_{k}$ are the quadratic forms on $T^{k}$ that are "push-forwards" of the form $G$ restricted to $T^{[k]}(X)$ under the quotient homomorphisms $T^{[k]}(X) \rightarrow T^{k}(X)$ and where $\operatorname{sym} \underline{G}_{k}$ are symmetrisations of this form under all permutations of the $2 k$ "indices".

To make sense of this, regard the quadratic forms $\underline{G}_{k}$ on the symmetric powers $T^{k}(X)$ as $2 k$-linear forms on $X, \underline{G}_{k}\left(\tau_{1}, \ldots, \tau_{2 k}\right)$ for $\tau_{1}, \ldots, \tau_{2 k} \in T(X)$. These forms are non-symmetric and $\operatorname{sym} \underline{G}_{k}$ denotes the corresponding symmetric $2 k$-forms that can be seen as homogeneous polynomials of degrees $2 k$ on the tangent spaces $T_{x}(X)$, $x \in X$.

In general, such "fully symmetric" graded forms $G^{\circ}=\oplus_{k} G^{k}$ on $\oplus_{k} T_{x}^{k}(X)$, $x \in X$, are "the same" as polynomials of degrees $2 l$ on the tangent space $T_{x}(X)$ with all monomials of even degrees $2 k, k=1,2, \ldots, l$.

Higher Isometric Immersion Problem. Let $G^{\circ}=\left\{G_{x}^{\circ}\right\}_{x \in X}$ be a family of "fully symmetric" graded forms $G_{x}^{\circ}=\bigoplus_{k} G_{x}^{k}$ on $\bigoplus_{k} T_{x}^{k}(X), x \in X$, that can be thought of as polynomials of degrees $2 l$ on the tangent spaces $T_{x}(X)$ with all monomials of even degrees $2 k, k=1,2, \ldots, l$.

A $C^{l}$-smooth map $f: X \rightarrow \mathbb{R}^{q}$ is called $G^{\circ}$-isometric, if $G^{\circ}$ equals the symmetrisation of the form on the jet bundle $T^{[l]}(X)$ that is induced by the $l$-jet of $f$ from the above form $H$ on $T^{[l]}\left(\mathbb{R}^{q}\right)$,

$$
G^{\circ}=\operatorname{sym}_{\oplus}\left(D^{[l]} f\right)^{\star}(H)=\bigoplus_{k}^{l} \operatorname{sym} \underline{G}_{k} .
$$

Observe that $\underline{G}_{2}$ here equals the second fundamental form of the immersion $X \stackrel{f}{\leftrightarrow} \mathbb{R}^{q}$; accordingly, $G^{\circ}$-isometric immersions for $l=2$ are exactly "immersions with prescribed curvatures" of $\mathbf{2}$ on p. 176. Similarly, these $f$ for $l=3$ can be christened isometric immersions with prescribed curvatures and torsions.

(Symmetrisation of the induced form $\left(D^{[l]} f\right)^{\star}(H)$ is needed to avoid a conflict with the higher order Gauss formulas that express the non-symmetric part of $\underline{G}_{k}$ in terms of derivatives of $\underline{G}_{k-1}$, see 3.1.5 in [35] and references therein.)

Let $C^{r_{k}}$ be the smoothness classes of $G^{k}$ as functions on $T^{k}(X)$.

What are $G^{\circ}$-isometric $C^{r}$-maps $f: X \rightarrow \mathbb{R}^{q}$ for a given $r \geq l$ ?

78 In particular, what is the minimal $q$ such that such an $f$ exists for all $G^{\circ}$ in a given (multi)regularity class $\left\{C^{r_{k}}\right\}$ ?

On Higher Dimensional Inheritance. One may define an inherently $m$ dimensional structure of order $l$ on a manifold $X$ as a (marked) subset in the space of $l$-jets at $0 \in \mathbb{R}^{m}$ of smooth maps $\mathbb{R}^{m} \rightarrow X$ or, more generally, as a subset in the natural linear span of this space of jets as was done for $m=1$.

The prominent examples of these for $m=2$ and $l=1$ are the symplectic structure and the conformal Riemannian structure.

One knows in this respect that symplectic immersions between symplectic (necessarily even dimensional) manifolds $X^{n} \rightarrow Y^{q}$ satisfy the $h$-principle except for the case $q=n$.

What is more significant is that the (suitably defined) $h$-principle for symplectic embeddings, that holds for $q<n+2$, is violated for $q=n+259$

\footnotetext{
${ }^{59}$ Apparently, there is a hidden network of deep connections between "symplectically rigid" and "symplectically flexible", the currently available knowledge of which is exposed by Eliashberg in his survey [26].
} 
The inherent 2-dimensionality is prominent for conformal structures on surfaces but is less visible for $X^{n}$ if $n \geq 3$, where conformal immersions have been studied in Riemannian geometry from a different perspective (see, e.g., [16] and references therein).

It is known $[50] 60$ that

Riemannian $C^{a n}$-manifolds $X^{n}$ are locally conformally $C^{a n}$-immersible

to $\mathbb{R}^{s_{n}-1}, s_{n}=\frac{n(n+1)}{2}$.

On the other hand, the following question seems wide open:

When can a $C^{\infty}$-Riemannian metric $g$ on $X$ be decomposed to a sum of

$p$ conformally flat $C^{\infty}$-metrics $g_{\nu}$, or, differently but in the same spirit, as

$$
g=\sum_{\nu=1}^{p} \psi_{\nu}^{2} g_{\nu}
$$

where $\psi_{\nu}$ are $C^{\infty}$-functions and where $g_{\nu}$ are quadratic forms that are induced by $C^{\infty}$-maps $f_{\nu}: X \rightarrow \mathbb{R}^{q_{\nu}}$ for given $p$ and dimensions $q_{\nu}$ ?

(This generalises Nash's $\sum \psi_{\nu} d \phi_{\nu}^{2}$-decomposition where $q_{\nu}=1$.)

What we know about symplectic immersions generalises, although perfunctorily, to exterior $m$-forms (see 3.4.1 in [35]) that are are inherently $m$-dimensional structures of degree 1; this may extend to "isometric immersions" of exterior forms on $T^{[l]}(X)$ as well. But we have no idea how to approach (or even to properly mathematically formulate) the following:

Semi-Philosophical Question. Are there inherently $m$-dimensional versions of the inherently one-dimensional techniques that have been developed for Riemannian isometric immersions?

\subsection{Unclassified Problems and Conjectures.}

1. Paradoxes of Low Regularity of Nash and of Kolmogorov and Arnold.

Simple counting of parameters gives a fair idea for (maybe conjectural) solvability or rather non-solvability of differential equations in the $C^{\infty}$-category, but it may break down at low regularity as it happens for isometric immersions produced by Nash's $C^{1}$-construction.

Apparently, counting parameters also motivated Hilbert in his conjecture of nonrepresentability of general functions in $n$ variables by superpositions of continuous functions in $(n-1)$ variables that was disproved by Kolmogorov in 1956-1957.

Can the constructions of $C^{1}$-isometric maps by Nash and Kuiper and of superpositions of $C^{0}$-functions by Kolmogorov and Arnold be brought to a common ground?

Hilbert's vision was justified, up to some degree, by Vitushkin for superpositions of $C^{r}$-functions for $r>0$ (see the surveys [72] and [73]).

But neither in the case of non-linear PDEs, e.g., for isometric $C^{r}$-immersions $X^{n} \rightarrow \mathbb{R}^{q(n, r)}$, nor for superposition problems for functions has the following found a comprehensive answer.

QUESTION. Where does, smoothness-wise versus dimension-wise, the demarcation line lay that separates what counting parameters predict and what low regularity constructions may deliver?

2. Genericity Problems. It is often, if not always, painfully difficult to show that a particular "object" $o_{0}$, e.g., a smooth function or a map between manifolds,

\footnotetext{
${ }^{60} \mathrm{I}$ owe this reference to Deane Yang.
} 
satisfies certain property that is known to be satisfied by generic, in some sense, $o$ in the class $O$ of $o_{0}$.

For instance Hilbert's conjecture remains open in the $C^{\infty}$-category.

Can (branches of the) solutions $x=x\left(a_{i}\right)$ of algebraic equations $\sum_{i=0}^{d} a_{i} x^{i}=$ 0 of high degree $d$ be locally represented (away from their ramification loci) by superpositions of $C^{\infty}$-smooth functions in two variables.

(This is also unknown for all $C^{r}, r \geq 1$, where additional difficulty comes from section 4.3 item 1 above.)

The two, mutually dual corresponding Riemannian problems are as follows:

Cartan Rigidity Conjecture. Local $C^{\infty}$-immersions $f: X^{n} \rightarrow \mathbb{R}^{q}$ are generically $C^{\infty}$-rigid for $q<s_{n}=\frac{n(n+1)}{2} 61$

81 MicroflexibiLity Conjecture. The metric inducing operator $f \mapsto g=$ $(D f)^{\star}\left(\sum_{j} d y_{j}^{2}\right)$ is infinitesimally invertible at generic $C^{\infty}$-immersions $f: X \rightarrow \mathbb{R}^{q}$ for $q>s_{n}$. Consequently, the sheaves of isometric $C^{\infty}$-immersions are, generically, microflexible. (Microflexibilty is a very strong violation of rigidity.)

3. Global Uniqueness Problems. The rigidity problem is accompanied by the global, as well as local, uniqueness/non-uniqueness questions.

Under what conditions is a $C^{\infty}$-smooth (real analytic) isometric immersion $(X, g) \rightarrow \mathbb{R}^{q}$ unique, and when, on the contrary, are there many "drastically different" smooth maps $X \rightarrow \mathbb{R}^{q}$ that induce the same metric on $X$ ?

Counting parameters suggests that if $q<\frac{k-1}{k} s_{n}$, then $k$-tuples of $C^{\infty}$-maps $f_{i}: X^{n} \rightarrow \mathbb{R}^{q}, i=1, \ldots, k$, that induce equal metrics on $X^{n}$ must be rare, but if $q>\frac{k-1}{k} s_{n}, s_{n}=\frac{n(n+1)}{2}$, then there must be lots of them.

A specific question here can be formulated as follows.

Under what assumptions on $n, k, q, r$, can all $k$-tuples of continuous maps from all smooth $n$-manifolds, $X \rightarrow \mathbb{R}^{q}$, be approximated by $k$-tuples of $C^{r}$-maps that induce equal metrics on $X$ ?

If $r=1$, then the Nash-Kuiper theorem delivers such approximating maps starting from $q=n+1$ (see section 3.3) but this seems unlikely for large $r$ and, if $k=2$, for $q<<\frac{s_{n}}{2}$ which, albeit looks easy, does not seem to be known even for $q=n+1$.

On the other hand,

if $q \geq \frac{s_{n}}{2}+2 n+2$, then the pairs of $C^{a n}$-maps $f_{1}, f_{2}: X \rightarrow \mathbb{R}^{q}$ that induce equal metrics on $X$ are dense in the space of all pairs of continuous maps $X \rightarrow \mathbb{R}^{q}$.

(These questions are addressed in [34] and 3.3.4 in [35.)

4. Regularity and Approximation Problems. Riemannian metrics induced by $C^{r}$-maps for $r \geq 2$ are "nearly $C^{r}$ " rather than mere $C^{r-1}$ : in some local (harmonic) coordinates they are in the Hölder class $C^{r-1, \alpha}$ for all $\alpha<1$ by a $1980-1982$ theorem of Nikolaev, Jost, and Karcher [51].

This suggests that smoothness of a metric must be defined in a coordinate free manner and also leads to the following

$84 C^{1,1}$-CONJECTURE. Every Riemannian manifold $X$ with bounded sectional curvatures in the sense of Alexandrov admits an isometric $C^{1,1}$-embedding (i.e., with Lipschitz first derivatives) to some Euclidean (infinite dimensional Hilbertian?) space.

\footnotetext{
${ }^{61}$ Known results and references can be found in the paper by Berger, Bryant and Griffiths (1983, 4]).
} 
Embeddings of Alexandrov Spaces Problem. Is there a class of isometric embedding and of their regularity properties characteristic for Alexandrov spaces with curvatures bounded from below?

(See [5] for an account of related regularity results.)

Let $X$ be a closed simply connected Riemannian $C^{a n}$-manifold of dimension $n \geq 1$, and let $f: X \rightarrow \mathbb{R}^{q}$ be an isometric $C^{\infty}$-immersion.

Under what conditions on $X$ and on $q$ do the following hold?

A. The map $f$ is real analytic.

B. The map $f$ can be $C^{\infty}$-approximated by real analytic maps.

C. The map $f$ can be approximated by real analytic maps $X \rightarrow \mathbb{R}^{q+m} \supset \mathbb{R}^{q}$ for a given $m=1,2, \ldots$.

It seems easy - this must be in the literature - that A is valid for $q=n+1$ and $n \geq 3$, but the case $n=2$ is, probably, unknown.

In general, A is expected for $q<s_{n}=\frac{n(n+1)}{2}$ and "generic" $X$ and, possibly, even for $q=s_{n}$. But it is hard to formulate a smart genericity condition here. Yet, the following properties may serve as guidelines for ruling out counterexamples, at least for "smallish" $q$, say for $q \leq 2 n-1$.

- $X$ admits no smooth topological immersion to $\mathbb{R}^{q-1}$;

- $X$ admits no isometric $C^{a n}$-immersion to $\mathbb{R}^{q-1}$;

- $X$ admits no local isometric $C^{a n}$-immersion to $\mathbb{R}^{q-1}$;

- $X$ does not metrically split or, more generally, it does not split to a warped product.

If $q<s_{n}$, these conditions are also favourable for B. On the other hand, it is not impossible that if $q>s_{n}$, then B holds for all $X$. But this is unknown even for $q \geq s_{n+2}$, where one exercises full control over free isometric immersions.

This leads us to $\mathrm{C}$ which is, for all $q$ :

* easily provable, with no compactness assumption on $X$, for $m=s_{n}+n$, with the help of generic bendings of $\mathbb{R}^{q}$ in $\mathbb{R}^{q+s_{n}+n} \supset \mathbb{R}^{q}$;

* a bit harder for $m=s_{n}$;

* realistic for $m \geq s_{n}-\sqrt{n / 2}$;

* plausible for $m=s_{n}-n+1$;

* not impossible for $m=n$.

5. Elementary Isometric Embeddings. Do "simple" manifolds $X$ admit "elementary" isometric embeddings to Euclidean spaces, or is the "high transcendence" inherent in Nash's constructions unavoidable?

For instance, one counts symmetric Riemannian spaces as "simple" as well as whatever one obtains from simple manifolds by "simple" constructions, e.g., by warped products, conformal changes of metrics, Riemannian fibrations.

It is harder to say what elementary embeddings are. One cannot limit oneselfat least not in the Riemannian category - to algebraically defined embeddingsthink of the hyperbolic metric algebraically expressed in the "projective model" -but one may search for embeddings taken from a "small" (finite dimensional?) pool of functions, e.g., solutions of integrable (non-linear) PDEs with algebraic "coefficients" 62

\footnotetext{
${ }^{62}$ In his 2001 survey [5, Borisenko collects most known and many new results concerning such immersions; see also 66], [3, 6] and [4].
} 
The oldest in this game are isometric immersions of the hyperbolic spaces $H^{n}$ to $\mathbb{R}^{q}$ that are known to admit "simple" local isometric $C^{a n}$-immersions to $\mathbb{R}^{2 n-1}$ and where the standing conjectures are as follows:

[A] $H^{n}$ admits a proper isometric $C^{a n}$-embedding $f: H^{n} \rightarrow \mathbb{R}^{2 n}$

(but it is doubtful that there is a "simple" such $f$ ).

(For the moment, the existence of such isometric $C^{a n}$-immersions is proven only for $H^{n} \rightarrow \mathbb{R}^{6 n-6}$, which is done in 3.2 .2 of [35], by approximating Blanuša's $C^{\infty}$ embeddings with a use of a non-compact version of Nash's $C^{a n}$-implicit function theorem.)

[B] $H^{n}$ admits no isometric $C^{2}$-immersion to $\mathbb{R}^{2 n-1}$.

The latter was proven by Hilbert (1901) for $n=2$ and if $n \geq 3$, where the corresponding non-immersability result is known for complete non-simply connected manifolds with constant negative curvatures by the work of Moore (1972), Xavie (1985), and Nikolayevsky (1998) 63

Hilbert's theorem was generalised by Efimov for surfaces with negative curvatures as follows.

The Jacobians of the Gauss maps $G: X^{2} \rightarrow S^{2}$ of all complet 64 non-compact immersed $C^{2}$-surfaces $X^{2} \hookrightarrow \mathbb{R}^{3}$ satisfy

$$
\inf _{x \in X}|\operatorname{Jac}(G(x))|=0 .
$$

Does this remain true for hypersurfaces $X^{n} \hookrightarrow \mathbb{R}^{n+1}$ if $n>2$ ?

See 2 for some results and conjectures in this direction.

90 What is the minimal $q$ such that:

[a] the Euclidean space $\mathbb{R}^{q}$ (or the sphere $S^{q}$ ) contains an algebraic submanifold of dimension $n$ with the induced Riemannian metric of constant negative curvature?

[b] some (all?) closed $n$-manifolds with constant negative curvature admit isometric $C^{a n}$-immersions to $\mathbb{R}^{q}$ ?

[c] the space $\mathbb{R}^{q}$ contains a closed $C^{\infty}$-submanifold of dimension $n$ with strictly negative curvature?

Is conformal flatness of (hyperbolic) manifolds relevant for their isometric immersion?

In general,

91 do $C^{\infty}$-smooth conformally flat manifolds of dimension $n$ admit local isometric $C^{\infty}$-immersions to $\mathbb{R}^{2 n+1}$ ?

\section{"FlAT" QUESTIONS}

92 What is the minimal $q$ depending on the topology of a flat manifold $X$ and/or on its holonomy group, such that $X$ admits an isometric $C^{r}$-immersion to $\mathbb{R}^{q}$ ? For instance,

93 what is the minimal $q$, such that $\left(\mathbb{R}^{n} \backslash 0\right) /\{ \pm 1\}$ isometrically $C^{r}$-embeds to $\mathbb{R}^{q}$ ?

94 Does this $q$ depend on $r$ for $r \geq 2$ ?

\footnotetext{
${ }^{63}$ Futher results and references can be found in [11.

${ }^{64}$ This means with complete induced Riemannian metric.
} 
There is no apparent geometric obstruction for isometric $C^{a n}$-immersions of flat manifolds to $\mathbb{R}^{q}$ for $q \geq 2 n 65$ but there may be topological obstructions for $q<3 n$ for non-parallelisable manifolds. None is visible, however, for $q \geq 3 n$, and although no general construction for immersion of flat manifolds is currently available for $q<s_{n+1}=\frac{(n+1)(n+2)}{2}$, one may safely conjecture that

if $q \geq 3 n$, then all strictly curve-shortening maps between Riemannian flat manifolds, $X^{n} \rightarrow Y^{q}$, admit approximations by isometric real analytic ones.

On the other hand, strong constraints on the shape of $C^{2}$-isometric immersions $X^{n} \rightarrow Y^{q}$, in particular, lower bounds on their sizes do exist for $q \leq 2 n-166$

For instance, the unit ball $B_{\text {Euc }}^{n} \subset \mathbb{R}^{n}$ admits no isometric $C^{3}$-immersion $\left(C^{2}\right.$ ?) to a ball of radius $<1$ in $\mathbb{R}^{2 n-1}$ by a 1952 theorem by Chern and Kuiper [13] (which, as I recall, needs $C^{3}$ ).

This seems to imply the similar result for $C^{2}$-approximately isometric immersions between arbitrary Riemananian manifolds.

More generally, let $Y=(Y, \tilde{g})$ be a compact $C^{2}$-smooth Riemannian manifold of dimension $q \leq 2 n-1$, and let $f_{i}$, be a sequence of $C^{\infty}$-immersions from the $n$-ball $B^{n}$ to $Y$, such that the induced metrics $g_{i}, i=1,2, \ldots$, on $B^{n}$ satisfy the following:

- the sectional curvatures of $g_{i}$ are uniformly bounded,

$$
\left|\kappa\left(g_{i}\right)\right| \leq C_{0}<\infty \text { for all } i
$$

- the distances $r_{i}$ from the center $0 \in B^{n}$ to its boundary measured in metrics $g_{i}$ are separated from zero,

$$
r_{i} \geq \rho_{0}>0 \text { for all } i
$$

- the volumes of $\left(B\left(n, g_{i}\right)\right)$ are also separated from zero.

Then the diameters of the images $f_{i}(X) \subset Y$ measured with $\tilde{g}$ are also separated from zero,

$$
\operatorname{diam}_{Y}\left(f_{i}(X)\right) \geq \delta_{0}>0 \forall i=1,2,3, \ldots
$$

$60 \%$ Proof. Let $x_{i} \in X$ be a point that is "not too close" to the boundary where the norm of the external curvature of $X \stackrel{f i}{\leftrightarrow} Y$ is "approximately maximal" 67 call it $K_{i}\left(x_{i}\right)$, and rescale $Y$ at the points $y_{0}=f\left(x_{i}\right)$ by $\left(Y \sim Y_{i}\right)=\left(Y, \lambda_{i} \tilde{g}\right)$ for some constants $\lambda_{i} \rightarrow \infty$ such that $K_{i} / \lambda_{i}^{2}$, which are curvatures of $X \stackrel{f_{i}}{\rightarrow} Y$ after the rescaling, are very small, yet away from zero.

The manifolds $Y_{i}$ converge to $\mathbb{R}^{q}$ and the geometries of the $\lambda_{i}$-rescaled $\lambda^{-1}$-balls in $\left(X, g_{i}\right)$ converge to the standard Euclidean ball $B=B_{\mathrm{Euc}}^{n}$, while a subsequence of maps $f_{i}$ converges to an isometric $C^{1}$-immersion $f_{\infty}: B \rightarrow \mathbb{R}^{q}$ with bounded extrinsic curvatures.

It is (almost) obvious that this map is $C^{1, \alpha}$, for all $\alpha<1$, probabaly even $C^{1,1}$ and the proof reduces to the following

CONJECTURE. The Chern-Kuiper theorem remains valid for isometric $C^{1, \alpha<1}$ _ immersions $B^{n} \rightarrow \mathbb{R}^{q \leq 2 n-1}$ with bounded extrinsic curvatures.

\footnotetext{
${ }^{65}$ Certain obstructions for $q<2 n$, some of which are applicable to compact flat $n$-manifolds minus $m \leq n$ small balls are presented in 3.2.1 in [35] 1986; also see [5].

${ }^{66}$ The best result of this kind for $n=2$ is Burago's isoperimetric inequality; see [12] and 3.2.3 in 35 .

${ }^{67}$ This a standard game in the rescaling game we play.
} 
Question. Are there instances of curvature related geometric constraints on the shape of $C^{2}$-approximately isometric immersions $X^{n} \rightarrow Y^{q}$ for $q \geq 2 n$ ?

Are there instances of curvature related geometric constraints on the shape of $C^{2}$-approximately isometric immersions $X^{n} \rightarrow Y^{q}$ for $q \geq 2 n$ ?

\section{ACKNOWLEDGMENT}

The author thanks Michael Albanese who helped with the final editing of the text and corrected my English.

\section{About THE AUthor}

Misha Gromov is a Permanent Member of IHES and professor of mathematics at the Courant Institute of New York University. His many awards include the Wolf Prize, the Kyoto Prize, and the Abel Prize.

\section{REFERENCES}

[1] A. Akopyan, PL-analogue of Nash-Kuiper theorem, preliminary version (in Russian): http://www.moebiuscontest.ru/files/2007/akopyan.pdf www.moebiuscontest.ru, (2007).

[2] V. Alexandrov, On a differential test of homeomorphism, found by N. V. Efimov (in Russian) Contemporary Problems of Mathematics and Mechanics (Sovremennye Problemy Matematiki i Mekhaniki), 6, no. 2 (2011), 18-26.

[3] Yu. Aminov, The geometry of submanifolds, Gordon and Breach Science Publishers, Amsterdam, 2001. MR 1796237

[4] E. Berger, R. Bryant, and P. Griffiths, The Gauss equations and rigidity of isometric embeddings, Duke Math. J. 50 (1983), no. 3, 803-892, DOI 10.1215/S0012-7094-83-05039-1. MR714831

[5] A. A. Borisenko, Isometric immersions of space forms in Riemannian and pseudoRiemannian spaces of constant curvature (Russian, with Russian summary), Uspekhi Mat. Nauk 56 (2001), no. 3(339), 3-78, DOI 10.1070/RM2001v056n03ABEH000393; English transl., Russian Math. Surveys 56 (2001), no. 3, 425-497. MR1859723

[6] A. A. Borisenko, Intrinsic and Extrinsic Geometry of Multidimensional Submanifolds, Ekzamen, 2003 (in Russian).

[7] Yu. F. Borisov, $C^{1, \alpha}$-isometric immersions of Riemannian spaces (Russian), Dokl. Akad. Nauk SSSR 163 (1965), 11-13. MR0192449

[8] Yu. F. Borisov, Irregular surfaces of the class $C^{1, \beta}$ with an analytic metric (Russian, with Russian summary), Sibirsk. Mat. Zh. 45 (2004), no. 1, 25-61, DOI 10.1023/B:SIMJ.0000013011.51242.23; English transl., Siberian Math. J. 45 (2004), no. 1, 19-52. MR.2047871

[9] R. L. Bryant, P. A. Griffiths, and D. Yang, Characteristics and existence of isometric embeddings, Duke Math. J. 50 (1983), no. 4, 893-994, DOI 10.1215/S0012-7094-83-05040-8. MR.726313

[10] Vincent Borrelli, S. Jabrane, F. Lazarus, and B. Thiber, Isometric embeddings of the square at torus in ambient space, Electronic Research Announcements in Mathematical Sciences Volume 15, Pages 8-16, (2008).

[11] D. Brander, Results related to generalizations of Hilbert's non-immersibility theorem for the hyperbolic plane, Electron. Res. Announc. Math. Sci. 15 (2008), 8-16. MR2372896

[12] Yu. D. Burago, Isoperimetric type inequalities in the theory of surfaces of bounded exterior curvature (Russian), Zap. Naučn. Sem. Leningrad. Otdel. Mat. Inst. Steklov. (LOMI) 10 (1968), 203 pp. (errata inside back cover). MR0243465

[13] S.-s. Chern and N. H. Kuiper, Some theorems on the isometric imbedding of compact Riemann manifolds in euclidean space, Ann. of Math. (2) 56 (1952), 422-430. MR0050962

[14] C. J. S. Clarke, On the global isometric embedding of pseudo-Riemannian manifolds, Proc. Roy. Soc. London Ser. A 314 (1970), 417-428. MR0259813

[15] Sergio Conti, Camillo De Lellis, László Szekelyhidi Jr., $h$-Principle and Rigidity for $C^{1, \alpha_{-}}$ Isometric Embeddings, arXiv:0905.0370, (2009). 
[16] M. Dajczer, Submanifolds and isometric immersions, Mathematics Lecture Series, vol. 13, Publish or Perish, Inc., Houston, TX, 1990. Based on the notes prepared by Mauricio Antonucci, Gilvan Oliveira, Paulo Lima-Filho and Rui Tojeiro. MR 1075013

[17] G. D'Ambra, Nash $C^{1}$-embedding theorem for Carnot-Carathéodory metrics, Differential Geom. Appl. 5 (1995), no. 2, 105-119, DOI 10.1016/0926-2245(95)00010-2. MR.1334838

[18] G. D'Ambra and M. Datta, Isometric $C^{1}$-immersions for pairs of Riemannian metrics, Asian J. Math. 6 (2002), no. 2, 373-384, DOI 10.4310/AJM.2002.v6.n2.a8. MR 1928635

[19] G. D'Ambra and A. Loi, A symplectic version of Nash $C^{1}$-isometric embedding theorem, Differential Geom. Appl. 16 (2002), no. 2, 167-179, DOI 10.1016/S0926-2245(02)00067-0. MR.1893907

[20] G. D'Ambra and A. Loi, Non-free isometric immersions of Riemannian manifolds, Geom. Dedicata 127 (2007), 151-158, DOI 10.1007/s10711-007-9173-5. MR2338523

[21] M. Datta, Partial isometries of a sub-Riemannian manifold, Internat. J. Math. 23 (2012), no. 2, 1250043, 17, DOI 10.1142/S0129167X12500437. MR2890477

[22] H. Davenport, Analytic methods for Diophantine equations and Diophantine inequalities, The University of Michigan, Fall Semester, vol. 1962, Ann Arbor Publishers, Ann Arbor, Mich., 1963. MR0159786

[23] R. De Leo, A note on a conjecture of Gromov about non-free isometric immersions. arXiv:0905.0928 [math.DG], (2009).

[24] Jean-Pierre Demailly, Proof of the Kobayashi conjecture on the hyperbolicity of very general hypersurfaces, arXiv:1501.07625, (2015).

[25] V. L. Dol'nikov and R. N. Karasev, Dvoretzky type theorems for multivariate polynomials and sections of convex bodies, Geom. Funct. Anal. 21 (2011), no. 2, 301-318, DOI 10.1007/s00039011-0109-8. MR 2795510

[26] Y. Eliashberg, Recent advances in symplectic flexibility, Bull. Amer. Math. Soc. (N.S.) 52 (2015), no. 1, 1-26, DOI 10.1090/S0273-0979-2014-01470-3. MR3286479

[27] Y. Eliashberg and N. Mishachev, Introduction to the h-principle, Graduate Studies in Mathematics, vol. 48, American Mathematical Society, Providence, RI, 2002. MR1909245

[28] A. Friedman, Local isometric imbedding of Riemannian manifolds with indefinite metrics, J. Math. Mech. 10 (1961), 625-649. MR0125544

[29] A. Friedman, Isometric embedding of Riemannian manifolds into Euclidean spaces, Rev. Modern Phys. 37 (1965), 201-203. MR0179740

[30] F. Forstnerič, Oka manifolds: from Oka to Stein and back (English, with English and French summaries), Ann. Fac. Sci. Toulouse Math. (6) 22 (2013), no. 4, 747-809, DOI 10.5802/afst.1388. With an appendix by Finnur Lárusson. MR3137250

[31] R. E. Greene, Isometric embeddings, Bull. Amer. Math. Soc. 75 (1969), 1308-1310. MR.0253240

[32] R. E. Greene, Isometric embeddings of Riemannian and pseudo-Riemannian manifolds., Memoirs of the American Mathematical Society, No. 97, American Mathematical Society, Providence, R.I., 1970. MR0262980

[33] R. E. Greene and H. Jacobowitz, Analytic isometric embeddings, Ann. of Math. (2) 93 (1971), 189-204. MR0283728

[34] M. L. Gromov, Smoothing and inversion of differential operators (Russian), Mat. Sb. (N.S.) 88(130) (1972), 382-441. MR0310924

[35] M. Gromov, Partial differential relations, Ergebnisse der Mathematik und ihrer Grenzgebiete (3) [Results in Mathematics and Related Areas (3)], vol. 9, Springer-Verlag, Berlin, 1986. MR864505

[36] M. Gromov, Oka's principle for holomorphic sections of elliptic bundles, J. Amer. Math. Soc. 2 (1989), no. 4, 851-897, DOI 10.2307/1990897. MR1001851

[37] M. Gromov, Sign and geometric meaning of curvature (English, with English and Italian summaries), Rend. Sem. Mat. Fis. Milano 61 (1991), 9-123 (1994), DOI 10.1007/BF02925201. MR.1297501

[38] M. Gromov, Carnot-Carathéodory spaces seen from within, Sub-Riemannian geometry, Progr. Math., vol. 144, Birkhäuser, Basel, 1996, pp. 79-323. MR1421823

[39] M. L. Gromov and V. A. Rohlin, Imbeddings and immersions in Riemannian geometry (Russian), Uspehi Mat. Nauk 25 (1970), no. 5 (155), 3-62. MR0290390 
[40] I. E. Gulamov and M. N. Smolyakov, Submanifolds in five-dimensional pseudo-Euclidean spaces and four-dimensional FRW universes, Gen. Relativity Gravitation 44 (2012), no. 3, 703-710, DOI 10.1007/s10714-011-1301-8. MR2899290

[41] M. Günther, Zum Einbettungssatz von J. Nash (German), Math. Nachr. 144 (1989), 165-187, DOI 10.1002/mana.19891440113. MR.1037168

[42] M. Günther, Isometric embeddings of Riemannian manifolds, Proceedings of the International Congress of Mathematicians, Vol. I, II (Kyoto, 1990), Math. Soc. Japan, Tokyo, 1991, pp. 1137-1143. MR 1159298

[43] R. S. Hamilton, The inverse function theorem of Nash and Moser, Bull. Amer. Math. Soc. (N.S.) 7 (1982), no. 1, 65-222, DOI 10.1090/S0273-0979-1982-15004-2. MR656198

[44] Q. Han and J.-X. Hong, Isometric embedding of Riemannian manifolds in Euclidean spaces, Mathematical Surveys and Monographs, vol. 130, American Mathematical Society, Providence, RI, 2006. MR2261749

[45] M. W. Hirsch, Immersions of manifolds, Trans. Amer. Math. Soc. 93 (1959), 242-276. MR.0119214

[46] J. Hong, Some New Developments of Realization of Surfaces to $\mathbb{R}^{3}$, ICM 2002, Vol. III, 1-3.

[47] L. Hörmander, The boundary problems of physical geodesy, Arch. Rational Mech. Anal. 62 (1976), no. 1, 1-52. MR0602181

[48] Norbert Hungerbühler and Micha Wasem, The One-Sided Isometric Extension Problem. arXiv:1410.0232, (2015).

[49] H. Jacobowitz, Extending isometric embeddings, J. Differential Geometry 9 (1974), 291-307. MR 0377773

[50] H. Jacobowitz and J. Moore, The Cartan-Janet theorem for conformal embeddings, Annals of Math., 116 (1982), 249-330

[51] J. Jost, Riemannian geometry and geometric analysis, 6th ed., Universitext, Springer, Heidelberg, 2011. MR2829653

[52] A. Källén, Isometric embedding of a smooth compact manifold with a metric of low regularity, Ark. Mat. 16 (1978), no. 1, 29-50, DOI 10.1007/BF02385981. MR499136

[53] N. H. Kuiper, On $C^{1}$-isometric imbeddings. I, II, Nederl. Akad. Wetensch. Proc. Ser. A. 58 = Indag. Math. 17 (1955), 545-556, 683-689. MR0075640

[54] M. McQuillan and M. Liam, Holomorphic curves on hyperplane sections of 3-folds, Geom. Funct. Anal. 9 (1999), no. 2, 370-392, DOI 10.1007/s000390050091. MR1692470

[55] B. Minemyer, Simplicial Isometric Embeddings of Indefinite Metric Polyhedra, arXiv:1211.0584v5 [math.MG], (2012).

[56] B. Minemyer, Isometric embeddings of polyhedra, ProQuest LLC, Ann Arbor, MI, 2013. Thesis (Ph.D.)-State University of New York at Binghamton. MR3192962

[57] H. Mirandola and F. Vitorio, Global Isometric Embeddings Mathematical Tripos, Part arXiv:1210.1812v2 [math.DG], (2014).

[58] H. Mirandola and F. Vitório, Global isometric embeddings of multiple warped product metrics into quadrics, Kodai Math. J. 38 (2015), no. 1, 119-134, DOI 10.2996/kmj/1426684445. MR 3323516

[59] S. Müller and V. Šverák, Convex integration for Lipschitz mappings and counterexamples to regularity, Ann. of Math. (2) 157 (2003), no. 3, 715-742, DOI 10.4007/annals.2003.157.715. MR.1983780

[60] N. Nadirashvili, Isoperimetric inequality for the second eigenvalue of a sphere, J. Differential Geom. 61 (2002), no. 2, 335-340. MR.1972149

[61] N. Nadirashvili and Y. Yuan, Improving Pogorelov's isometric embedding counterexample, Calc. Var. Partial Differential Equations 32 (2008), no. 3, 319-323, DOI 10.1007/s00526-0070140-7. MR 2393070

[62] J. Nash, $C^{1}$-isometric imbeddings, Ann. of Math. (2) 60 (1954), 383-396. MR0065993

[63] J. Nash, The imbedding problem for Riemannian manifolds, Ann. of Math. (2) 63 (1956), 20-63. MR0075639

[64] J. Nash, Analyticity of the solutions of implicit function problems with analytic data, Ann. of Math. (2) $\mathbf{8 4}$ (1966), 345-355. MR0205266

[65] A. Petrunin, On intrinsic isometries to Euclidean space, 2010, arXiv:1003.5621

[66] E. G. Poznyak and D. D. Sokolov, Isometric immersions of Riemannian spaces in Euclidean spaces, Algebra, Geometry, and Topology, vol. 15 (Itogi Nauki i Tekhniki), 1977. 
[67] J. Schwartz, On Nash's implicit functional theorem, Comm. Pure Appl. Math. 13 (1960), 509-530. MR0114144

[68] S. Smale, A classification of immersions of the two-sphere, Trans. Amer. Math. Soc. 90 (1958), 281-290. MR0104227

[69] S. Smale, The classification of immersions of spheres in Euclidean spaces, Ann. of Math. (2) 69 (1959), 327-344. MR0105117

[70] D. Spring, Convex integration theory, Monographs in Mathematics, vol. 92, Birkhäuser Verlag, Basel, 1998. Solutions to the $h$-principle in geometry and topology. MR 1488424

[71] D. Spring, The golden age of immersion theory in topology: 1959-1973. A mathematical survey from a historical perspective, Bull. Amer. Math. Soc. (N.S.) 42 (2005), no. 2, 163-180 (electronic), DOI 10.1090/S0273-0979-05-01048-7. MR2133309

[72] A. G. Vitushkin, Hilbert's thirteenth problem and related questions (Russian, with Russian summary), Uspekhi Mat. Nauk 59 (2004), no. 1(355), 11-24, DOI 10.1070/RM2004v059n01ABEH000698; English transl., Russian Math. Surveys 59 (2004), no. 1, 11-25. MR2068840

[73] A. G. Vituškin and G. M. Henkin, Linear superpositions of functions (Russian), Uspehi Mat. Nauk 22 (1967), no. 1 (133), 77-124. MR0237729

[74] C. Voisin, On some problems of Kobayashi and Lang, https://webusers.imj-prg.

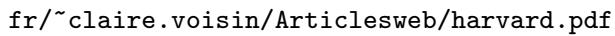

[75] D. Yang, Gunther's proof of Nash's isometric embedding theorem, https://archive.org/ stream/arxiv-math9807169/math9807169_djvu.txt

Institut des Hautes Études Scientifiques, Bures-sur-Yvette, France; And Courant Institute for Mathematical Sciences, New York University, New York 